\title{
Sequence-based Protein Interaction Site Prediction using \\ Computer Vision and Deep Learning
}

By

\author{
Aishwarya Purohit
}

A thesis submitted to the Faculty of Graduate and Postdoctoral Affairs in partial fulfillment of the requirements for the degree of

\section{Master of Applied Science}

in Biomedical Engineering with Specialization in Bioinformatics

Ottawa-Carleton Institute for Biomedical Engineering

Department of Systems and Computer Engineering

Carleton University

Ottawa, Ontario, Canada

May 2021 


\section{Abstract}

Computational prediction of protein-protein interaction (PPI) from protein sequence is important as many cellular functions are made possible through PPI. The Protein Interaction Prediction Engine (PIPE) software suite was developed at Carleton University for such predictions. This thesis aims to conduct a thorough performance assessment of the PIPE-Sites predictor through the use of a large high-quality set of known PPI sites. The results show that PIPE-Sites has relatively low accuracy even after retuning the inherent hyperparameters of the method. Furthermore, PIPE-Sites are shown to be ineffective when applied to similarity-weighted score data. Thus, three new sequence-based methods of predicting PPI sites are proposed and evaluated, including the Panorama, BrightSpot, and ClusterNet methods. The new methods leverage similarityweighted score data to further increase performance. Ultimately, ClusterNet significantly outperforms the other methods over two different performance metrics when evaluated on both human and yeast data PPI site data. 


\section{Extended Abstract}

Many biological functions in the cell are made possible through physical proteinprotein interaction (PPI). Therefore, computational prediction of PPI from protein sequence is an important goal of bioinformatics. The Protein Interaction Prediction Engine (PIPE) software suite was developed at Carleton University to predict PPI and the sub-sequence within each interacting protein that supports the physical interaction (PIPE-Sites). One important recent advancement in PIPE is the similarityweighted score (SW-score), which normalizes the weight of evidence for a PPI, based on the background frequency of each amino acid window among the proteome. However, the SW-score has not been leveraged by the PIPE-Sites software to improve PPI site (subsequence) prediction. Also, PIPE-Sites was originally trained on a relatively small set of known interaction sites, limiting its accuracy. Finally, prediction performance of the original PIPE-Sites method used a "distance metric" calculation that failed to penalize under-prediction of the true interaction site.

This thesis makes the following contributions: 1) a larger high-quality set of known PPI are assembled and used to conduct a thorough performance assessment of PIPESites. It is demonstrated that PIPE-Sites has relatively low accuracy, when measured using an appropriate metric introduced here, even after retuning the inherent hyperparameters of the method. 2) PIPE-Sites is shown to be ineffective when applied to SW-score data (landscapes). 3) Three new sequence-based methods of predicting PPI sites are proposed and evaluated, including the panorama method, the brightspot method, and ClusterNet (a deep-learning-based method). The 
ClusterNet method is shown to significantly outperform all other methods over two different performance metrics. Furthermore, the new methods are able to leverage SW-score data to further increase performance. Lastly, the experimental results on human PPI site data are validated on yeast data. 


\section{Acknowledgements}

I would first like to thank my supervisor, Prof. James Green, for his exceptional support, patience, and guidance throughout the entire research journey. His insightful feedback pushed me, sharpen my thinking and brought my work to achievable outcomes.

I would also like to thank all the members of the Carleton University Bioinformatics Group for their collaboration and providing inspiration for my research. My appreciation goes out to all the authors of PIPE, whose work served as the foundation for this study. I am grateful to the lab members for their constant support and assistance at different stages of the research work.

Finally, I want to thank my family, my parents, my brother and especially my partner, Shrinivas Acharya, whose continual love and encouragement was critical to overall success. 


\section{Table of Contents}

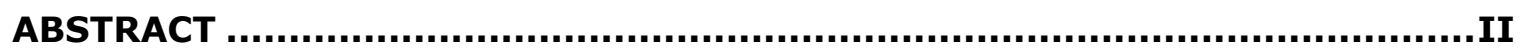

EXTENDED ABSTRACT ........................................................................ III

ACKNOWLEDGEMENTS.,.

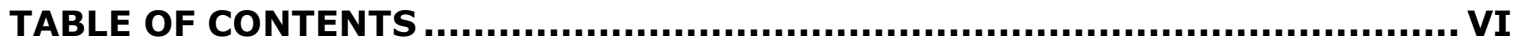

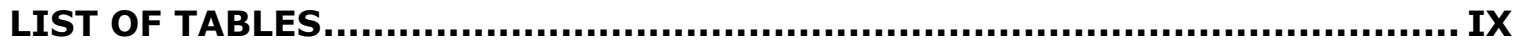

LIST OF FIGURES

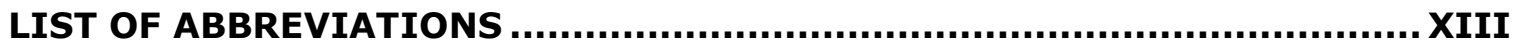

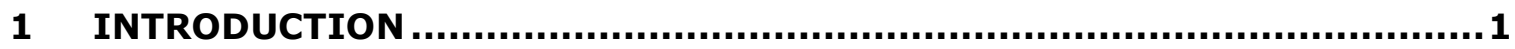

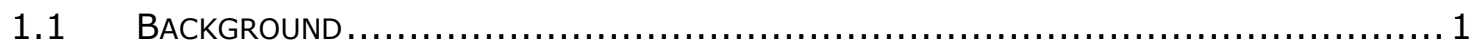

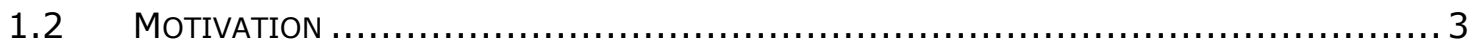

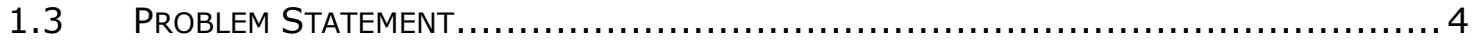

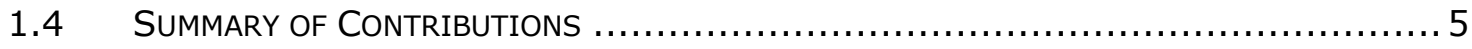

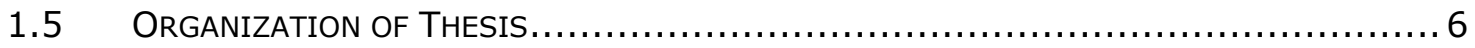

2 LITERATURE REVIEW................................................................... 7

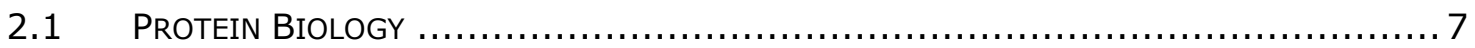

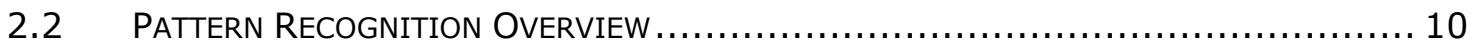

2.2.1 Image Pattern Recognition ......................................... 11

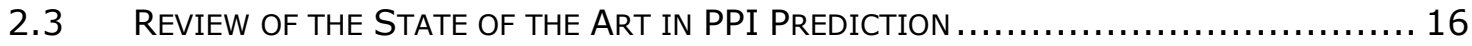

2.3.1 Computational PPI Prediction ............................................ 16

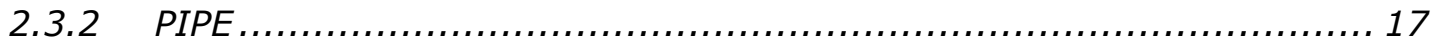

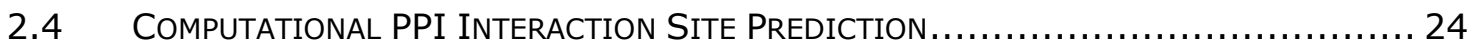

2.4.1 Interaction Site Prediction .............................................. 24

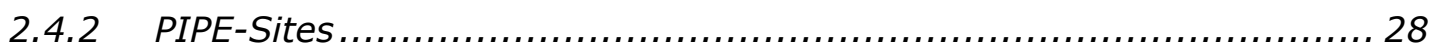

2.4.3 Walk Algorithm Explanation.......................................... 29

2.4.4 Validation of Prediction PPI-Sites: Distance Measure...................... 30

2.4.1 Box-Plot distribution .................................................. 32 


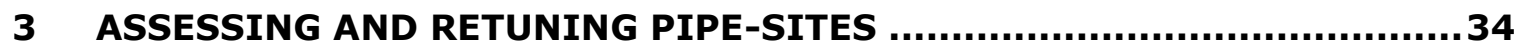

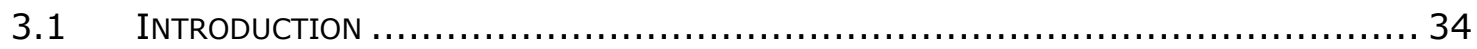

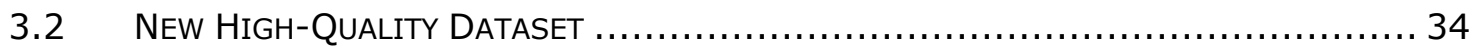

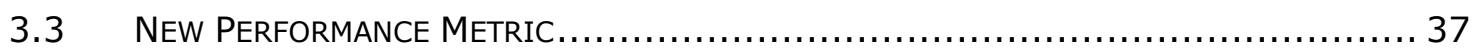

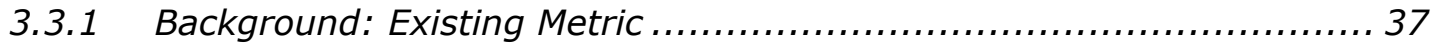

3.3.2 Proposal ............................................................. 38

3.3.3 Conclusion ........................................................ 39

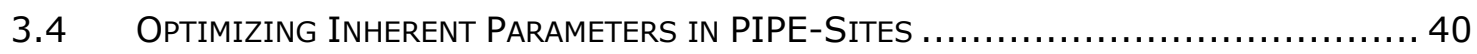

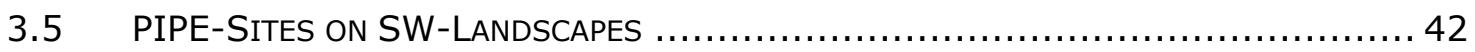

4 INTRODUCING NEW PPI-SITES DETECTION TECHNIQUES ...................45

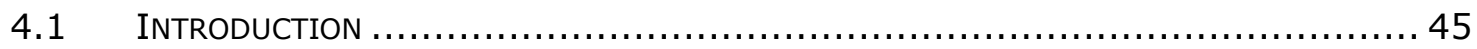

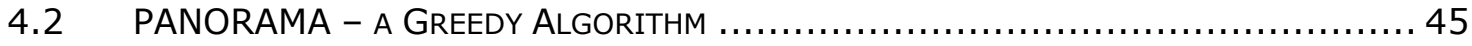

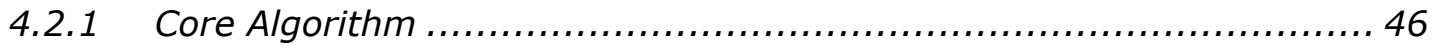

4.2.2 Parameters Optimization .............................................. 53

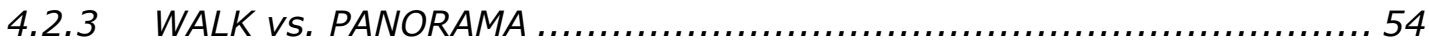

4.3 BRight-SPOT Detection - Classical Image Segmentation $\ldots \ldots \ldots \ldots \ldots \ldots \ldots \ldots$

4.3.1 Core Algorithm ..................................................... 56

4.3.2 Parameters Optimization........................................... 64

4.3.3 WALK vs. BRIGHT-SPOT Detection ................................. 66

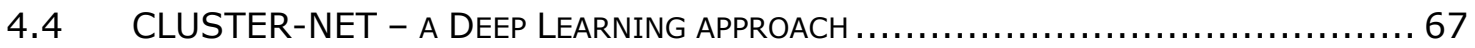

4.4.1 Core Algorithm ....................................................... 69

4.4.2 Parameter Configuration ................................................ 87

W.4.3 WALK vs. CLUSTER-NET ............................................ 89

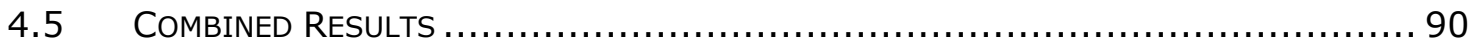

4.5.1 Box Plots .................................................................. 90

4.5.2 Statistical Tests ................................................... 92

4.5.3 AUC : Area Under the Curve ........................................ 95

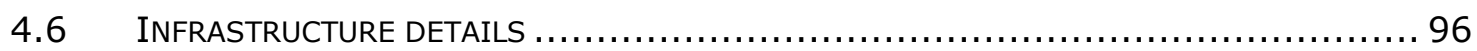

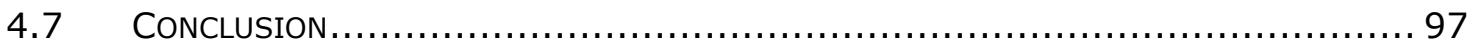


5 VALIDATION OF NEW PPI-SITE DETECTION TECHNIQUES ON YEAST ... 98

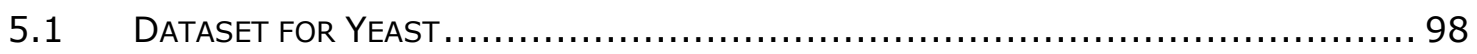

5.2 COMPARING VARIOUS TECHNIQUES ON YEAST DATASET $\ldots \ldots \ldots \ldots \ldots \ldots \ldots \ldots \ldots \ldots$

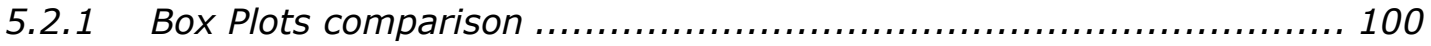

5.2.2 Statistical Tests ....................................................... 101

5.2.3 Area Under the Curve Performance .................................. 102

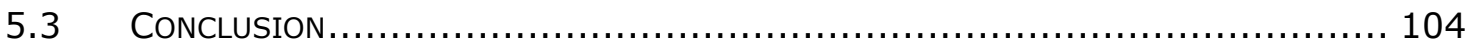

6 THESIS SUMMARY AND FUTURE RECOMMENDATIONS ...................... 105

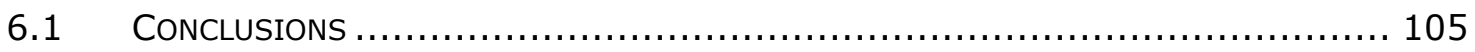

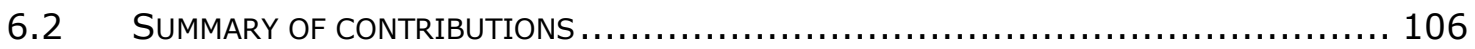

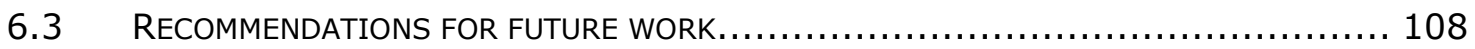

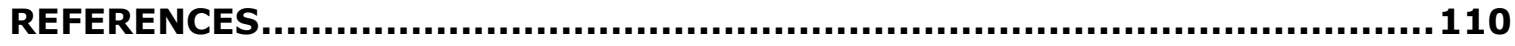




\section{List of Tables}

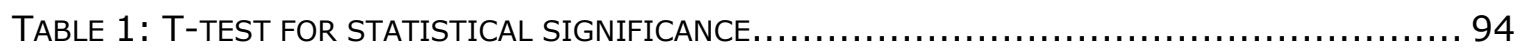

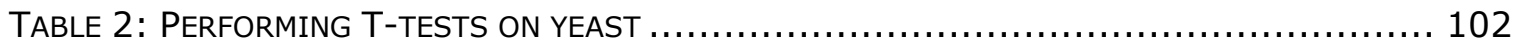




\section{List of Figures}

Figure 1: RePRESENTING DOMAIN-DOMAIN INTERACTION OF A PROTEIN-PAIR. HERE, PROTEIN-A, P63104, IS REPRESENTED ON THE LEFT, WITH ITS DOMAIN IN ORANGE, AND PROTEIN-B, P04792, IS REPRESENTED ON THE RIGHT, WITH DOMAIN COLORED IN RED .

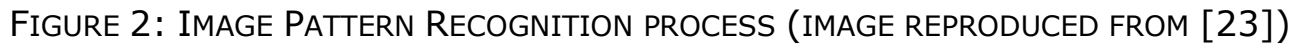
11

Figure 3: A simple multi layered Artificial Neural Network (FIGURE REPRODUCED FROM [33]) ...

Figure 4: A Simple Convolutional NeURAl NetWork - FOR HANDWRITTEN Digit RECOGNition

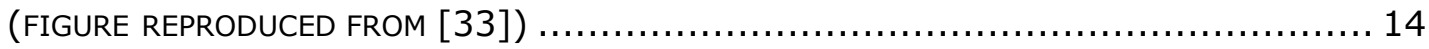

Figure 5: High LEVEL OVERVIEW OF PIPE (FIGURE REPRODUCED FROM [10]).... 18

FiguRE 6: OVERVIEW OF PIPE CORE ALGORITHM - THIS FIGURE EXAMINES THE FIRST SLIDING WINDOW OF 20 AMINO ACIDS IN EACH PROTEIN, HIGHLIGHTED IN GREEN. IN STEP 1, THE SIMILAR PROTEINS FOR THE WINDOWS HIGHLIGHTED IN GREEN ARE IDENTIFIED. IN STEP 2, THE KNOWN INTERACTIONS BETWEEN THE SIMILAR PROTEINS ARE COUNTED (THE KNOWN INTERACTIONS ARE HIGHLIGHTED IN BOLD AND DRAWN AS BLACK LINES BETWEEN PROTEINS IN THE TWO SETS). THIS COUNT IS ENTERED IN THE INTERACTION LANDSCAPE FOR THESE WINDOWS. THIS PROCESS IS THEN REPEATED FOR EACH PAIR OF WINDOWS (FIGURE REPRODUCED FROM [10]).... 19

FiguRe 7: SAMPLE PIPE LANDSCAPES FOR TWO POSITIVE PAIRS (TOP ROW) AND A NEGATIVE PAIR (BOTTOM ROW) (FIGURE REPRODUCED FROM [10]).... 20

FiguRE 8: SiMILARITY WEIGHTED (SW) METHOD FOR MODIFYING PIPE ORIGINAL-LANDSCAPES

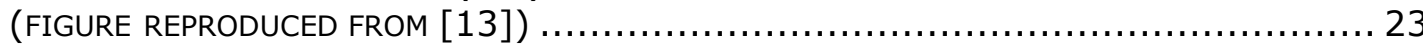

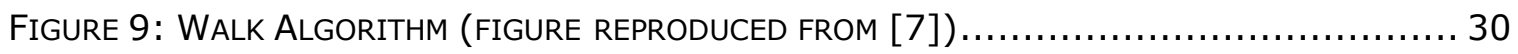

Figure 10: Distance Measure (DM) estimation (Figure Reproduced from [7]) $\ldots \ldots \ldots \ldots . \ldots 31$

FiguRE 11 : BOX PLOT AS A FIVE-NUMBER SUMMARY (FIGURE REPRODUCED FROM [61]) .......... 33

FiguRE 12 : BOX PLOT FOR A NORMAL DiSTRIBUTION (FIgURE REPRODUCED FROM [61]) ......... 33

Figure 13 : New High-Quality Dataset Extraction Pipeline - A Pipeline describing the PROCESS OF DATA EXTRACTION OF LAB-VERIFIED DOMAIN-DOMAIN INTERACTION SITES, AND HOW THAT IS USED TO PERFORM VALIDATIONS ON VARIOUS (EXISTING AND NEW) PIPE-SITES

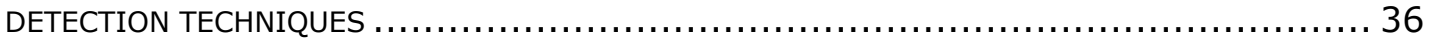

FiguRE 14 : INHERENT PARAMETER TUNING IN WALK (ORIGINAL METHOD FOR DETECTING PIPESITES) 40

FiguRE 15 : POOR PERFORMANCE OF WALK ALGORITHM WHEN APPLIED TO SW-LANDSCAPES (OVER NEW HIGH-QUALITY DATASET) 43

FIGURE 16 : SW-LANDSCAPE PARAMETER TUNING: PERFORMANCE OF WALK ON SW-LANDSCAPES

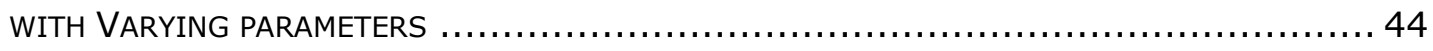


Figure 17 : WALK Site DETECTION - WALK CONSIDERS ONLY SCORES IN THE PEAK ROW AND

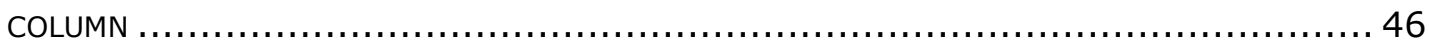

FigURE 18 : ILLUSTRATING DEFINITIONS OF UPPER-BAR, LOWER-BAR, RIGHT-BAR AND LEFT-BAR . 47

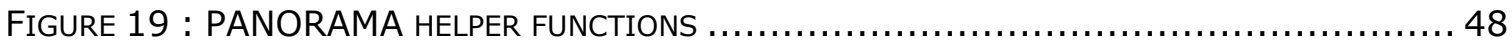

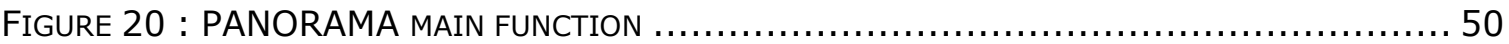

FIGURE 21 : PANORAMA TECHNIQUE - DESCRIBING THE PANORAMIC VIEW INSTEAD OF LINEAR

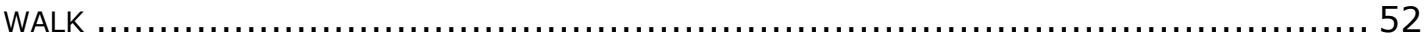

FIGURE 22 : PANORAMA SITE DETECTION - DOES NOT MISS DIAGONAL ENTRIES $\ldots \ldots \ldots \ldots \ldots \ldots . \ldots \ldots$

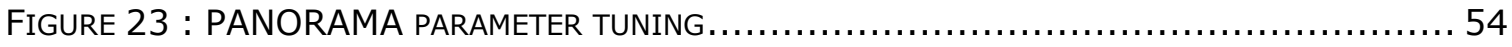

FiguRE 24 : COMPARING PANORAMA AND WALK ON SW-LANDSCAPES, WITH THEIR BEST

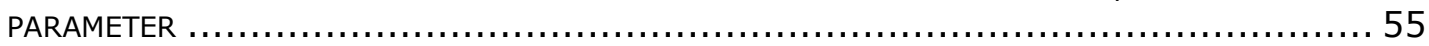

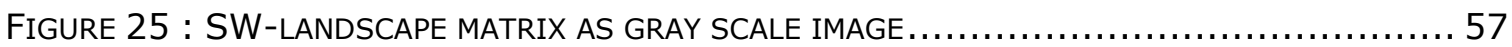

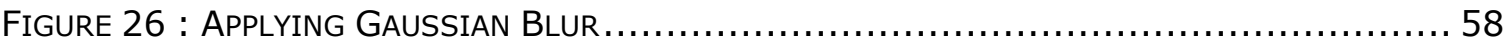

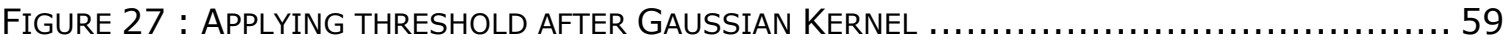

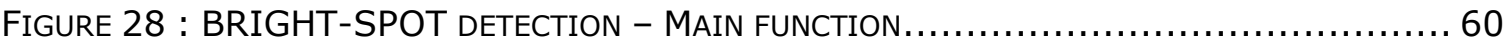

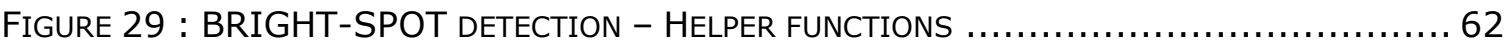

Figure 30 : CALCUlating Minimum EnClosing ReCtANGLES on CONNECTED BRight COMPONENTS

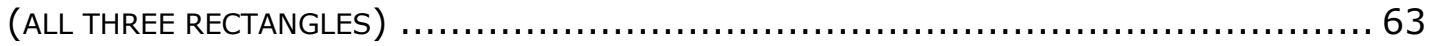

Figure 31 : Comparing BRIGHT-SPOT detection Sites With LAB VERIFIEd PPI-Site ....... 64

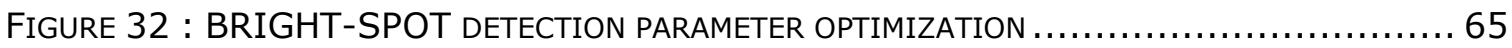

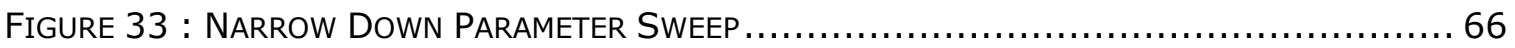

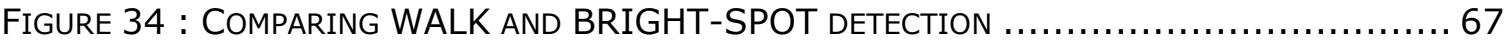

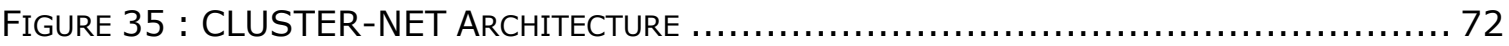

FiguRE 36 : SW-LANDSCAPE MATRIX AS 1 CHANNEL INPUT IMAGE (OF DIMENSION $H \times W \times 1$ ) $\ldots . . .74$

Figure 37 : PAssing inPut image to Convolution NeURAl Network $\ldots \ldots \ldots \ldots \ldots \ldots \ldots \ldots . \ldots \ldots$

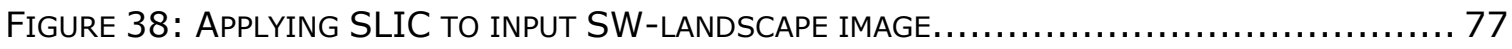

FiguRE 39: INPUT SW-LANDSCAPE TO CLUSTER-NET (RECREATION OF FIGURE 36 ) ............ 80

FIGURE 40: CLUSTER-NET TOP LARGEST FINAL SEGMENTS (HERE PLOTTING ONLY TOP 8 SEGMENTS

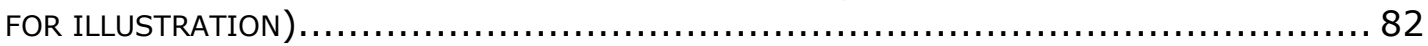




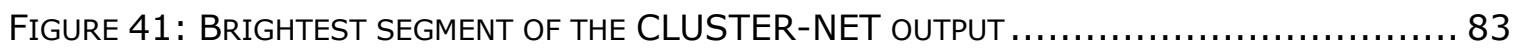

FigURE 42: REDUCING THE OVERFITTING OF CLUSTER-NET BY ADDING BLUR $\ldots \ldots \ldots \ldots \ldots \ldots . \ldots 4$

FigURE 43: Minimum ENCLOSING RECTANGLE FOR TOP 3 RANKED CONNECTED-COMPONENTS ..... 86

FiguRe 44: Comparing CLUSTER-NET PREDICTION Sites WITH LAB VERIFIEd PPI-Site ....... 87

FigURE 45: CLUSTER-NET PERFORMANCE FOR VARIOUS KERNEL SIZES $\ldots \ldots \ldots \ldots \ldots \ldots \ldots \ldots . \ldots 8$

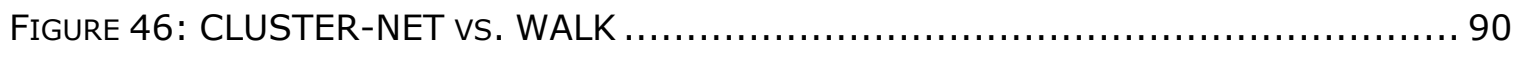

FigURE 47: BOX PLOT DISTRIBUTION OF ALL NEW TECHNIQUES COMPARED TO THE WALK .........91

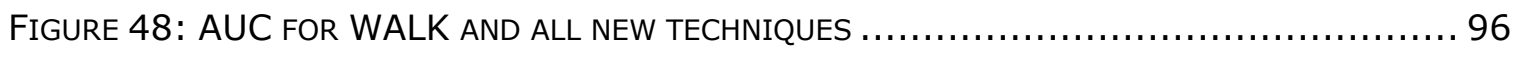

FIGURE 49: BOX-PLOT DISTRIBUTION OF YEAST PROTEIN-PAIRS FOR ALL TECHNIQUES WITH

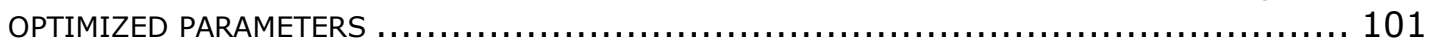

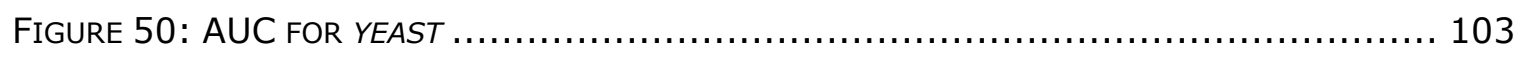

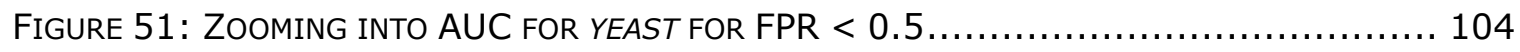




\section{List of Abbreviations}

\begin{tabular}{|c|c|}
\hline 3did & Database of 3D interacting domains \\
\hline AA & Amino Acids \\
\hline ADM & Absolute Distance Measure (new) \\
\hline ANN & Artificial Neural Network \\
\hline ANOVA & Analysis of Variance \\
\hline AUC & Area Under the Curve \\
\hline CNN & Convolutional Neural Network \\
\hline DM & Distance Measure (existing) \\
\hline GSCV & Grid Search Cross Validation \\
\hline $\mathrm{JI}$ & Jaccard Index \\
\hline PAM-Matrix & Point Accepted Mutation Matrix \\
\hline PBRs & Protein Binding Residues \\
\hline PIPE & Protein Interaction Prediction Engine \\
\hline PIPE-Sites & $\begin{array}{l}\text { Protein Interaction Prediction Engine - Interaction Site Prediction } \\
\text { Methods }\end{array}$ \\
\hline PPI & Protein-Protein Interaction \\
\hline PPI-Site & Protein-Protein Interaction site \\
\hline PR & Pattern Recognition \\
\hline ReLu & Rectified Linear Unit \\
\hline SLIC & Simple Linear Iterative Clustering \\
\hline SW-Score & Similarity - Weighted Score \\
\hline
\end{tabular}




\section{Introduction}

\subsection{Background}

Proteins are biomolecules that comprise the majority of the cellular machinery in any biological system. They are the most abundant organic molecules in living systems and are distinct in structure and function from other macromolecules [1]. Although their structure, similar to their function, varies enormously, all proteins are composed of one or more chains of amino acids (AA) [2]. A protein's primary structure is characterized by the arrangement of a gene that encodes the unique sequence of AA (of which there are 20 types) which are joined together by peptide bonds. A chain of amino acids can be referred to as a polypeptide sequence. Proteins carry out many cellular functions through physical protein-protein interactions (PPI) with other proteins [3]. Such PPI are essential to many cellular processes including the regulation of biochemical pathways, cellular motion, forming protein complexes, or carrying another protein. Therefore, determining PPI networks facilitates the elucidation of all these cellular pathways. Additionally, understanding how proteins interact, and evaluating the site of interaction for each protein, will provide information on complex networks, evolution, and human pathology [2].

The significance of understanding these PPIs led to the development of different experimental methods to detect and characterize such interactions including both experimental (in vivo or in vitro) and computational (in silico) strategies [1]. Experimental approaches, such as yeast two-hybrid and affinity purification followed by mass 
spectroscopy, tend to be expensive, labor intensive, time consuming and suffer from noise [4].

Therefore, computational techniques have been successfully used to predict highthroughput protein interaction data with high quality and accuracy [5]. Hence, using computational approaches for the prediction for PPIs are an effective alternative, particularly those that predict PPI based solely on the primary sequence of the two query proteins since three-dimensional protein structure is much more difficult to discern [6]. Given the increase in the availability of PPIs and validation dataset for variety of species, sequence-based computational prediction methods are originated [2].

Beyond the prediction of whether two proteins will interact, characterization of the amino acid subsequences that support the PPI provides important insights [7]. These so-called PPI "interaction sites" include all amino acids that form the physical interaction interface and also the surrounding amino acids that are required to support the PPI (e.g., surrounding amino acids may form the structural scaffolding for a binding pocket). A comprehensive understanding of these interaction sites can provide information for researchers and provide hypothesis-based starting points for drug design and diagnosis of diseases [2].

A variety of machine learning approaches have been used to predict PPIs and interaction sites, mostly based on combinations of classification, clustering and feature selection techniques [8]. Recently, sequence-based methods that directly extract information from a 
protein sequence have gained much attention. As an increasing amount of gold standard PPI data becomes available, their computation has become an important concern in bioinformatics [9].

\subsection{Motivation}

Interaction sites can be predicted from frequently occurring polypeptide sequences [7]. The underlying principle of sequence-based predictors is the conservation of subsequences (often referred to as domains and motifs) that appear in many PPI sites. That is, if a subsequence is known to enable a PPI in one or more proteins, and that same subsequence is observed in the query protein, this suggests that the query protein may also participate in PPI.

The protein-interaction prediction Engine (PIPE) is a high throughput, sequence-based method developed at Carleton University by the Bioinformatics Research Group [10]. This algorithm only requires the amino acid sequences of the proteins of interest, and a database of known PPIs. It works at high specificity and is highly computationally efficient, such that it can exhaustively analyze the binding patterns of all the proteins produced by any living organism. It uses a machine learning model to assign a probability of whether a pair of proteins will interact or not. In addition, the Protein Interaction Prediction Enginesites (PIPE-Sites) software goes beyond PPI prediction to also determine which parts of each protein, or "PPI (binding) sites", are responsible for the interaction [7]. 
PIPE-Sites was also developed at Carleton University. As per the data availability, PIPESites was trained using a relatively small dataset of gold standard PPI sites from yeast data. However, there are now much more PPI data available for different species. Additionally, the PIPE-Sites method is parameterized by a number of tunable hyperparameters in its core algorithm: the "walk" algorithm. These hyperparameters were optimized and validated using only small datasets. Furthermore, recent improvements to the PIPE algorithm have accounted for frequently occurring subsequences that are not informative

of protein interactions, leading to new similarity-weighted score-data (SW-score). The prediction of PPI interaction sites will likely benefit from leveraging these new SW-score data but may require retuning of the PIPE-sites hyperparameters or, indeed, replacement of the core algorithm.

\subsection{Problem Statement}

The overarching objective of this thesis is to improve PIPE-Sites prediction. This will be achieved by tackling three key problems:

\section{1) PIPE-Sites was trained and optimized using a small yeast dataset, using a flawed} performance metric. PIPE-Sites was trained using a relatively small dataset of PPI sites. It is expected that performance will be improved by using a larger and higher quality training set, made possibly by recent growth in databases containing experimental PPI interaction site data. Furthermore, the original performance metric fails to penalize under-prediction. Therefore, a more appropriate distance metric should be used. Lastly, the hyperparameters 
will be retuned using the new dataset and performance metric. Taken together, this should improve the performance of the original PIPE-Sites method.

2) PIPE-Sites is unable to leverage SW-Score data. Recently, PIPE has been improved by ruling out frequently occurring sequence patterns that are not strongly associated with protein-protein interaction. In this thesis, I will examine whether this "similarity-weighted score" approach can also improve PIPE-sites to more accurately predict binding sites. Using the enhanced training and evaluation pipeline developed above, it is conclusively determined if the original PIPE-Sites algorithm is suitable for predicting PPI sites from SWscore landscapes.

3) PIPE-Sites is a very simple algorithm that fails to leverage advances in image processing and machine learning, by treating $\mathrm{SW}$-landscapes just as scoring matrix. New methods are developed to predict PPI-sites from SW-landscapes, including those based on image processing and deep learning. All methods are developed and evaluated using the enhanced pipeline developed above.

\subsection{Summary of Contributions}

The new PPI-sites prediction validation pipeline is evaluated on $\sim 2500$ PPIs with known PPI interaction site information and a newly introduced performance metric. This new pipeline also improved the original PIPE-Sites through hyperparameter retuning by $\sim 25 \%$ measured over the new metric. Additionally, it is established that the original PIPE-Site algorithm is not suitable for analyzing SW-landscapes. Therefore, three new approaches for 
predicting PPI-sites from SW-landscape data were introduced, improving overall performance by $\sim 40 \%$ measured over the new metric.

\subsection{Organization of Thesis}

The remainder of the thesis consists of five chapters. Literature Review reviews the literature on different wet-lab and computational methods of PPIs prediction, leveraging both protein structure and sequence. It describes in detail about computational PPI prediction methods and provides an overview of the PIPE and PIPE-Sites algorithms since they underpin the contributions in this thesis. Assessing and Retuning PIPE-Sites explains the detailed implementation of the PIPE-Sites algorithms, proposes improvement in the original algorithm, and develops new performance metric as well. Introducing New PPISites Detection Techniques introduces three new techniques to improve PPI-Site prediction and evaluates them using the data and performance metric from Assessing and Retuning PIPE-Sites. We performed all the experiments so far on human protein-pairs. Validation of new PPI-Site detection techniques on Yeast discusses validations of experimentation and results of previous chapters, on yeast dataset. In the end, Thesis Summary and Future Recommendations summarizes the conclusions of this research work and provides recommendations for future work. 


\section{Literature Review}

This chapter first presents some background information regarding protein biology and machine learning. It then provides a critical literature review of the state of the art in protein-protein interaction site prediction.

\subsection{Protein Biology}

Amino acids are the building blocks of protein and have several properties that ultimately determine a protein's structure and function. Studying proteins are crucial for understanding an organism, although the number of unique proteins in a living being doesn't generally correspond to the complexity of the organism [11]. The size of the interactome - which is the collection of all PPI in an organism - has appeared to more directly correspond with the complexity of an organism [12]. Understanding these PPI in different organisms is consequently vital for evaluating their proper functioning. Nonetheless, just a small part of the true interactome is presently known [13].

While traditional "wet lab" experimental methods used for identifying PPIs are essential, they are both time-consuming and expensive. High throughput methods to determine many novel PPI exist, such as yeast two-hybrid, but they suffer from low accuracy and low repeatability [13] [14]. As there are more than 200 million potential pairs of proteins in human, experimentally confirming the total interactome for all these pairs is infeasible. Hence, computational techniques are frequently used to predict novel PPI to control biological methodologies. 


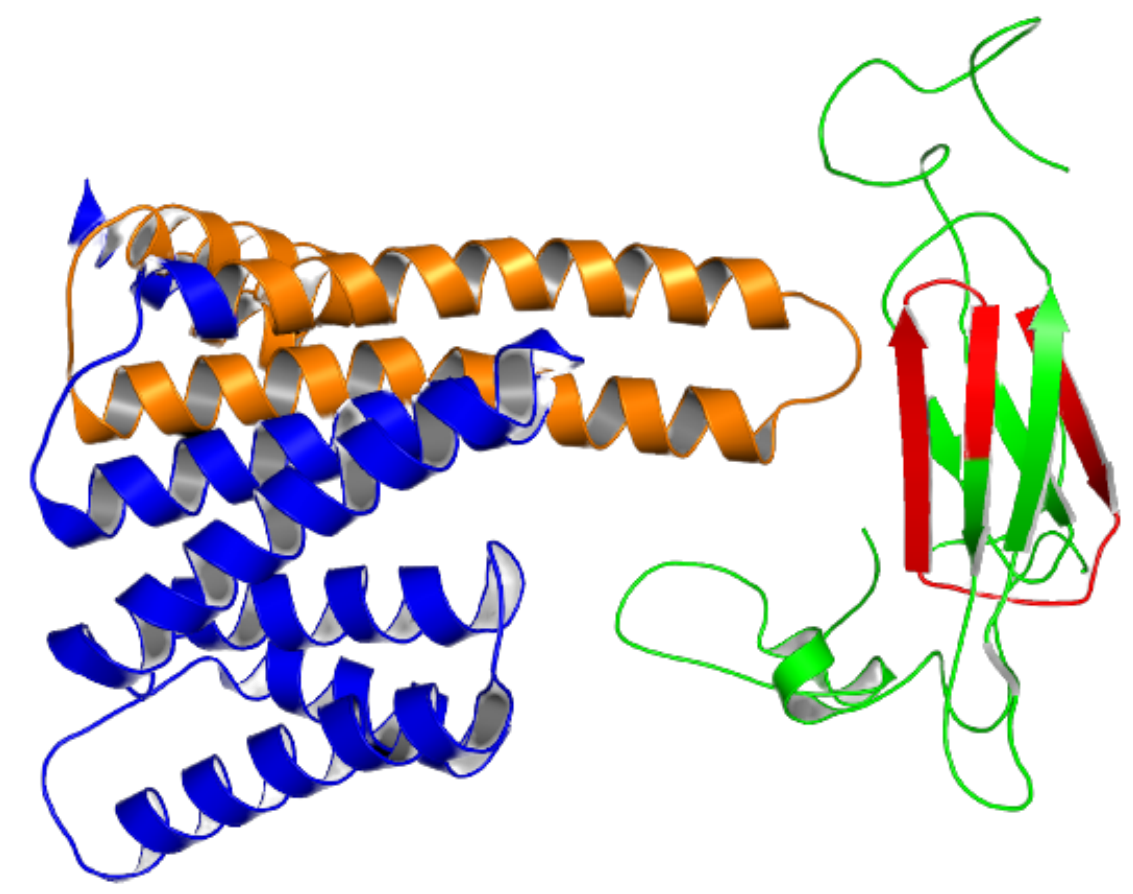

Figure 1: Representing domain-domain interaction of a protein-pair. Here, protein-A, P63104, is represented on the left, with its domain in orange, and protein-B, P04792, is represented on the right, with domain colored in red

Certain subsequences of amino acids form relatively independent and reusable components and appear repeatedly in multiple proteins. These subsequences are called domains, as shown in Figure 1. A large number of domains have been shown to be evolutionary conserved among proteins and are responsible for arbitrating these protein interactions [7]. Domain-domain interactions have been discovered utilizing association rules and are found to be responsible for various PPIs [15] [16]. These domains are also, often associated with providing specific functions to their proteins, such as DNA-binding, or mediating PPI. In fact, a number of PPI prediction methods focus on identifying such PPIrelated domains [17]. 
Many PPI prediction methods rely solely on the primary structure of the input proteins, enabling assessment of any pair of proteins without requiring 3D structural information. Computational PPI prediction techniques can generate huge protein interaction networks, that correspond to a map of all PPI within an organism's proteome. These protein interaction networks can give significant knowledge about aggregates and complexes for use in precision medicine [18]. However, even the most complete protein networks cannot reveal the hidden components that enable the binding of two proteins. PPIs are arbitrated by the actual physical binding on the outer surface of a fully folded protein. By analysing the binding sites that enable PPIs can provide information about any protein's role in a proteome.

Beyond predicting whether two proteins interact, the presence of known PPI-medicating domains can also elucidate the subsequence within a protein that enables the PPI. Such a region is known as the PPI interaction site. The PIPE-Sites PPI interaction site prediction method follows a similar principle but learns which subsequences (which are often shorter than complete domains) mediate PPI, directly from known interacting protein pairs [7]. There has been much research done on the subject of interaction site prediction [11]. Note that some methods attempt to identify the amino acid residues in each protein that are physically in contact with each other. These residues form the PPI "interface", which differs from the larger sequence that enables the PPI (i.e., the "PPI interaction site"). For example, other residues may be required to form the backbone structure that arranges for the PPI interface to have the correct structure. The PPI interaction site corresponds to a contiguous subsequence of a protein that is required to enable the PPI. Since the entire interaction site 
is required for the PPI, it will tend to be more highly conserved than other regions of the protein. This evolutionary conservation underpins sequence-based PPI site prediction methods, since the same conserved subsequence will appear in multiple proteins within the proteome (and potentially across species).

\subsection{Pattern Recognition Overview}

Pattern Recognition (PR) addresses the problem of classifying objects, often represented as numbers or vectors or as strings of symbols, known as features, into various categories [19]. The daunting task is to first synthesize, and second, effectively compute, the classification function that maps objects into categories, with the help of features. This classification function is also known as the predictor [20]. When the problem of the PR becomes too difficult to solve by hand-crafted algorithms, machine learning plays a central role. Most of times, such learning automatically synthesizes the classification function from a set of labelled examples. In such cases, it is called supervised learning [21]. When no prior knowledge is required, in the form of labelled examples, statistical information is extracted from the source in order to formulate the predictor. It is regarded as unsupervised learning [22].

The task of predicting the PPI-sites from the PIPE output, landscape matrix, is an example of part of pattern recognition. The original PIPE-Sites work introduced a hand-crafted algorithm, WALK, to predict PPI-sites. In this work we go beyond hand-crafted algorithm and use image segmentation techniques, which are categorised under the problem of image 
pattern recognition. We have also discussed various evaluation metrics to measure the performance of prediction of PPI-sites.

\subsubsection{Image Pattern Recognition}

Image Pattern Recognition (sometimes regarded as Image Processing), as the name suggests, is the problem of exploring how to recognize patterns in images [23]. An image pattern recognition system generally consists of multiple parts - source of image samples to be classified (for example a camera that takes images), a feature extraction mechanism that gains discriminative features from images for recognition, and finally the classification function. Sometimes there is another step before feature extraction, referred as preprocessing step, responsible for steps such as improving quality of image, removing unwanted area from the original image, enhancing some aspect of image important for feature extraction, etc. Figure 2 summarizes the process of image pattern recognition.

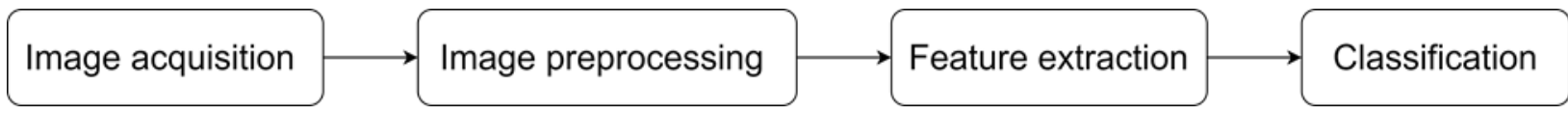

Figure 2: Image Pattern Recognition process (image reproduced from [23])

\subsubsection{Image Segmentation}

Image segmentation is a collection of techniques [24] that partition a given input image into several different regions, and each region shares common features, like similar texture, similar colors or semantically similar object [25]. The goal of image segmentation is to map every pixel of image to a meaningful group. Image segmentation is, most of the time, used to locate the pattern of interest, and find boundaries between objects. 
Effective evaluation of the quality of image segmentation is equally important. There are several traditional ways of evaluating the results of image segmentation, such as:

1. Pixel Accuracy [26] - It is the percentage of pixels that are classified in the correct segment. For our problem of predicting PPI-sites from an input landscape image, it is observed that average length of domain is small compared to the length of protein, making the area of PPI-site much smaller than total area of landscape image. Pixel-Accuracy is not a good metric in the case of such class imbalance problems, where a large part of the image is background. Hence, for an effective evaluation for such a class imbalance problem, we need another metric.

2. Jaccard Index (JI) [27] — also known as intersection over union. It is the fraction of overlapping area (or area of intersection) by the union of the area, hence its value ranges from 0 (no overlap) to 1 (complete overlap). It is one of the most used metrics.

3. Dice Coefficient [28] — It is like JI, but its value is equal to 2 times area of intersection divided by total number of pixels of both the segments. Its value also ranges from 0 to 1 and it is positively corelated to JI [29]. This means if Dice Coefficient stats that a classification function A is better than classification function B, at segmenting an image, then JI will also conclude the same, and vice versa.

Another metric to evaluate segmentation is Distance Measure, which is introduced in the original PIPE-Sites works [7] and is discussed later in the chapter. 


\subsubsection{Convolutional Neural Networks}

Figure 3 shows a simple three-layered Artificial Neural Network (ANN) [30] [31] with fully connected neurons. Convolutional Neural Networks (CNN) [32] [33] are analogous to traditional ANNs in that, they are comprised of neurons that self-optimise through learning. Each neuron still receives an input and performs an operation such as a scalar product followed by a non-linear function. From the input raw image vectors to the final output of the class score, the entire network still expresses a single perceptive score function (the weight). The last layer contains loss functions associated with the classes. All of the regular tips and tricks developed for traditional ANNs, such as back propagation, dropout etc. still apply. The only notable difference between CNNs and traditional ANNs is that CNNs are primarily used in the field of pattern recognition within images. This allows us to encode image-specific features into the architecture, making the network more suited for imagefocused tasks - whilst further reducing the parameters required to set up the model. 


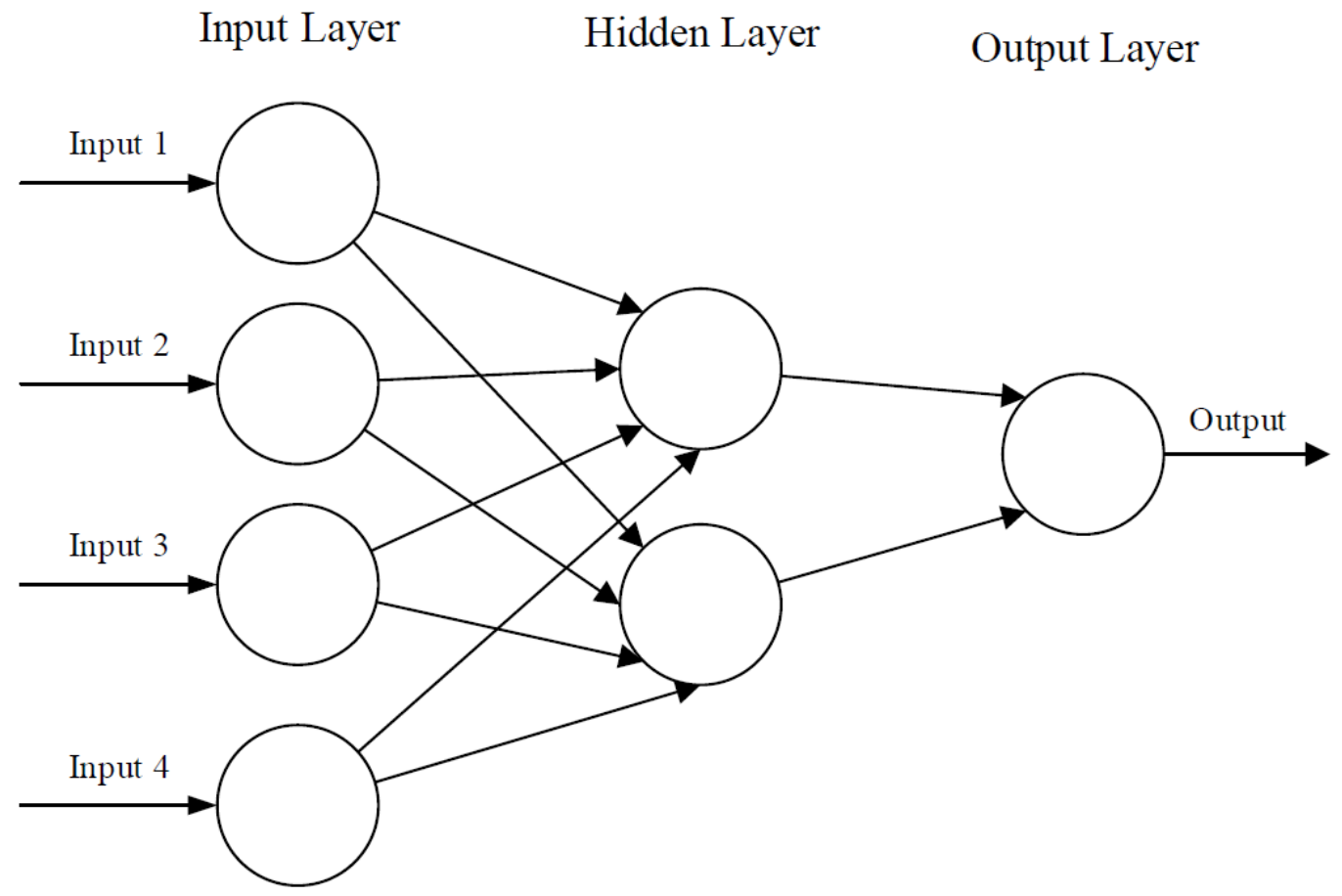

Figure 3: A simple multi layered Artificial Neural Network (figure reproduced from [33])

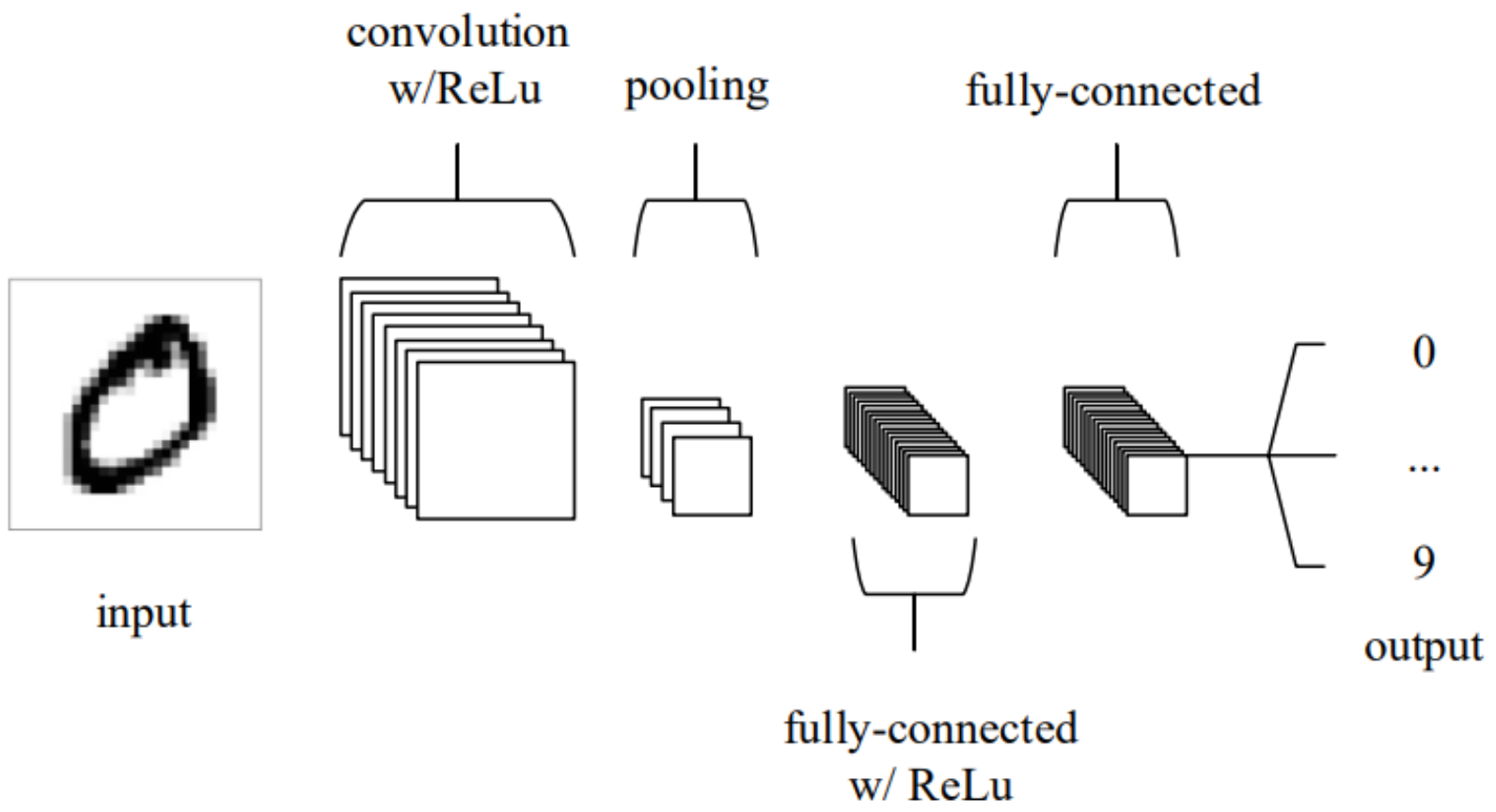

Figure 4: A simple Convolutional Neural Network - for handwritten digit recognition (figure reproduced from [33]) 
At a high level, CNNs are comprised of three types of layers. These are convolutional layers, pooling layers, and fully connected layers. When these layers are stacked, along with the input layer, a CNN architecture is formed. The basic functionality of the example CNN in Figure 4 can be broken down into following key areas -

1. The input layer holds the pixel values of the image.

2. The convolutional layer determines the output of neurons of which are connected to local regions of the input through the calculation of the scalar product between their weights and the region connected to the input volume. So far only a scalar transformation has been done.

3. A rectified linear unit, commonly shortened to ReLu [34], is often applied right after the convolutional layer. This aims to apply an elementwise activation function such as sigmoid [35] to the output of the activation produced by the previous layer.

4. The pooling layer then simply performs down-sampling along the spatial dimensionality of the given input, further reducing the number of parameters within that activation.

5. The fully connected layers perform the same duties found in the ANNs and attempt to produce class scores from the activations, to be used for classification. It is also suggested that ReLu may be used between these layers, as to improve performance [33].

Through these processes of transformation, CNNs are able to transform the original input, layer by layer, using convolutional and down-sampling techniques to produce class scores for classification and regression purposes. 


\subsection{Review of the State of the Art in PPI Prediction}

PPI support vital biological processes in cells [36]. Disruptions in those interactions can lead to diseases, including, but not limited to, various genetic disorders [37]. Identifying accurate cellular networks of PPI is, therefore, critical for understanding diverse living processes in cells. It is necessary for studies related to disorders and for drug discovery, as most common drug targets are proteins (like enzymes, ion channels, and receptors) [38]. There are many computational PPI prediction methods that make use of two types of information about proteins: structure-based and sequence-based methods.

\subsubsection{Computational PPI Prediction}

A number of PPI prediction methods make use of 3D structure information [39]. A popular method is 3D docking, where proteins are modeled in a three-dimensional space. Computational geometry is then used to determine if two or more proteins can fit together, based on various physical, molecular and genomic properties . Unfortunately, protein structure is not available for the majority of proteins, particularly for under-studied organisms. Structure-based predictions therefore suffer from lack of input data, which limits this type of method in terms of proteome-scale predictions. Additionally, these are computationally expensive processes that require large processing-time in order to reach a solution. Using modern molecular dynamics simulations to fully leverage 3D structure information is computationally expensive, and even on powerful distributed clusters, it can take months to complete. Methods such as PiSites [40], IntPred [41], Zang [42] require protein structure information as an input to predict PPI sites. 
As mentioned above, sequence-based PPI prediction methods seek to identify protein subsequences (domains or smaller conserved regions) that mediate PPI directly from the sequences of protein pairs known to interact. A recent sequence-based PPI prediction method, SPRINT (Scoring Protein Interactions) is conceptually similar to PIPE [43]; it depends on the assumption that underlies most sequence-based methodologies: a pair of proteins that are sequence-similar to a pair of interacting proteins has a higher likelihood to bind to each other. Unlike PIPE, sequence similarity is measured using a spaced-seed approach which makes SPRINT slightly faster [43]. Sub-sequences from an input pair of proteins are examined for similarity to known PPI and a prediction score is computed.

\subsubsection{PIPE}

While the previous section briefly reviewed some computational PPI prediction, this section provides a detailed description of the PIPE method, since it underpins the contributions made in this thesis. Within the domain of predicting protein-protein interactions completely from sequence, and by means of an algorithm that is altogether interpretable: enter the Protein Interaction Prediction Engine (PIPE) [44]. It is a technique developed here at Carleton University by the bioinformatics research members. PIPE predicts PPIs from sequences [10] [45] and by utilizing a data set of known protein interactions. Figure 5 illustrates high level overview of PIPE, detailed in the next section.

\subsubsection{Principle}

The approach works on the underlying hypothesis that across various proteins, almost similar local subsequences of amino acids would be yielding a 3D structure, and these 
structures will lead to forming interactions [13]. It works on the principle that the input candidate protein pair A-B are similar sequence to variety of protein pairs that are known to interact (e.g. X-Y, X-Z, Y-Z), then it postulates that proteins A and B would be likely to interact with each other [44]. Figure 5 illustrates a high-level overview of PIPE, where it is trained on a prior known list of interacting proteins and uses it to predict 'a score' for the likelihood of interaction for input protein pair.

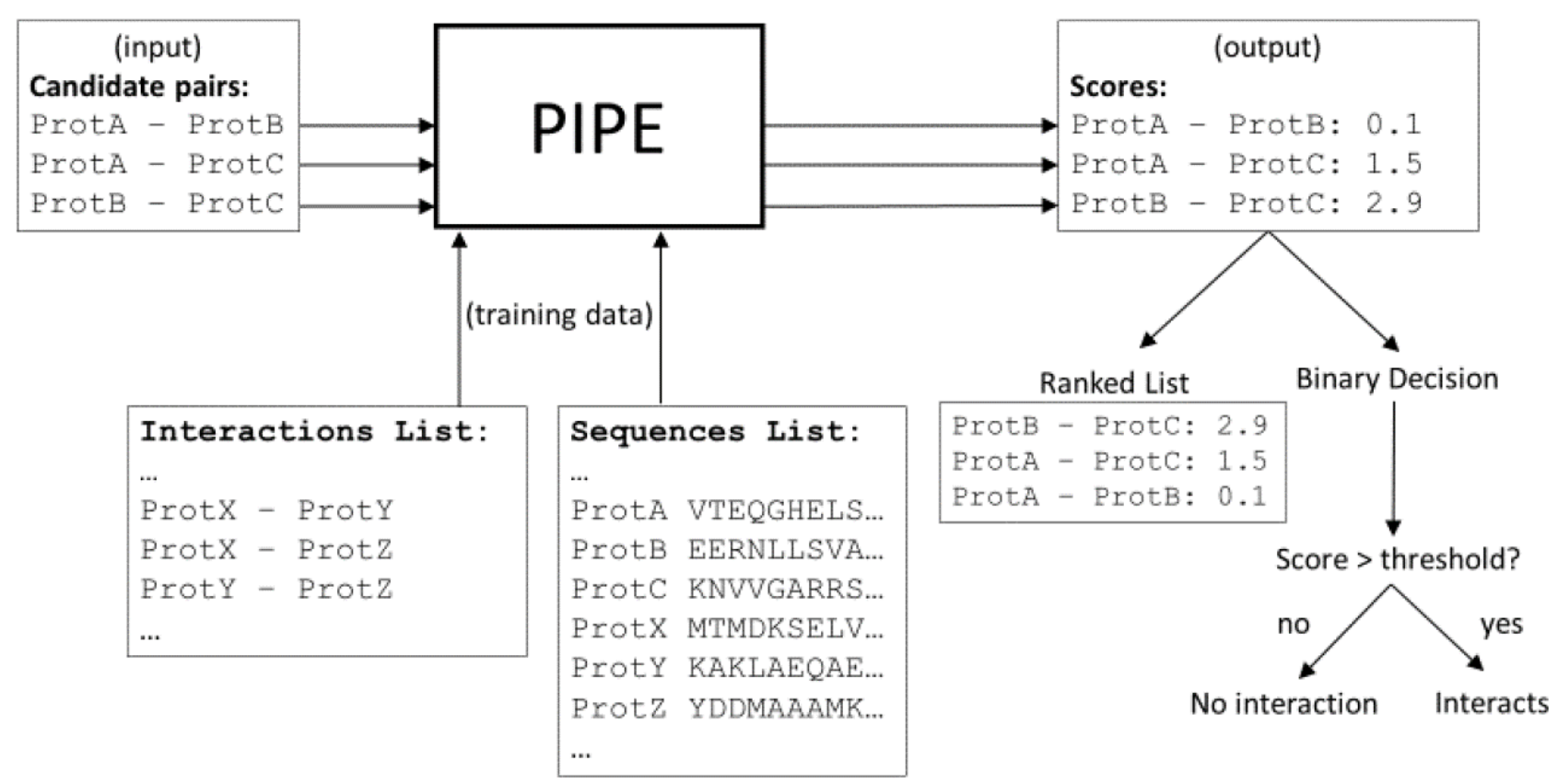

Figure 5: High level overview of PIPE (figure reproduced from [10]) 


\subsubsection{Core Algorithm}

In detail, PIPE compares the query proteins A and B using a 20-amino acid sliding window, which iterates by 1 amino acid across the lengths of both proteins A and B. Each window is compared against the full known interactome database available for that species [10]. The physicochemical similarity of one amino acid relative to another is translated by the Point Accepted Mutation (PAM) matrix [46]. This PAM120 score matrix is a reasonable way to quantify how physiochemically similar one amino acid is from another amino acid, in the context of being a member of the aforementioned interactions database. As an example, PIPE uses a PAM120 score threshold of 40 for Homo Sapiens and 35 for Saccharomyces cerevisiae [47].

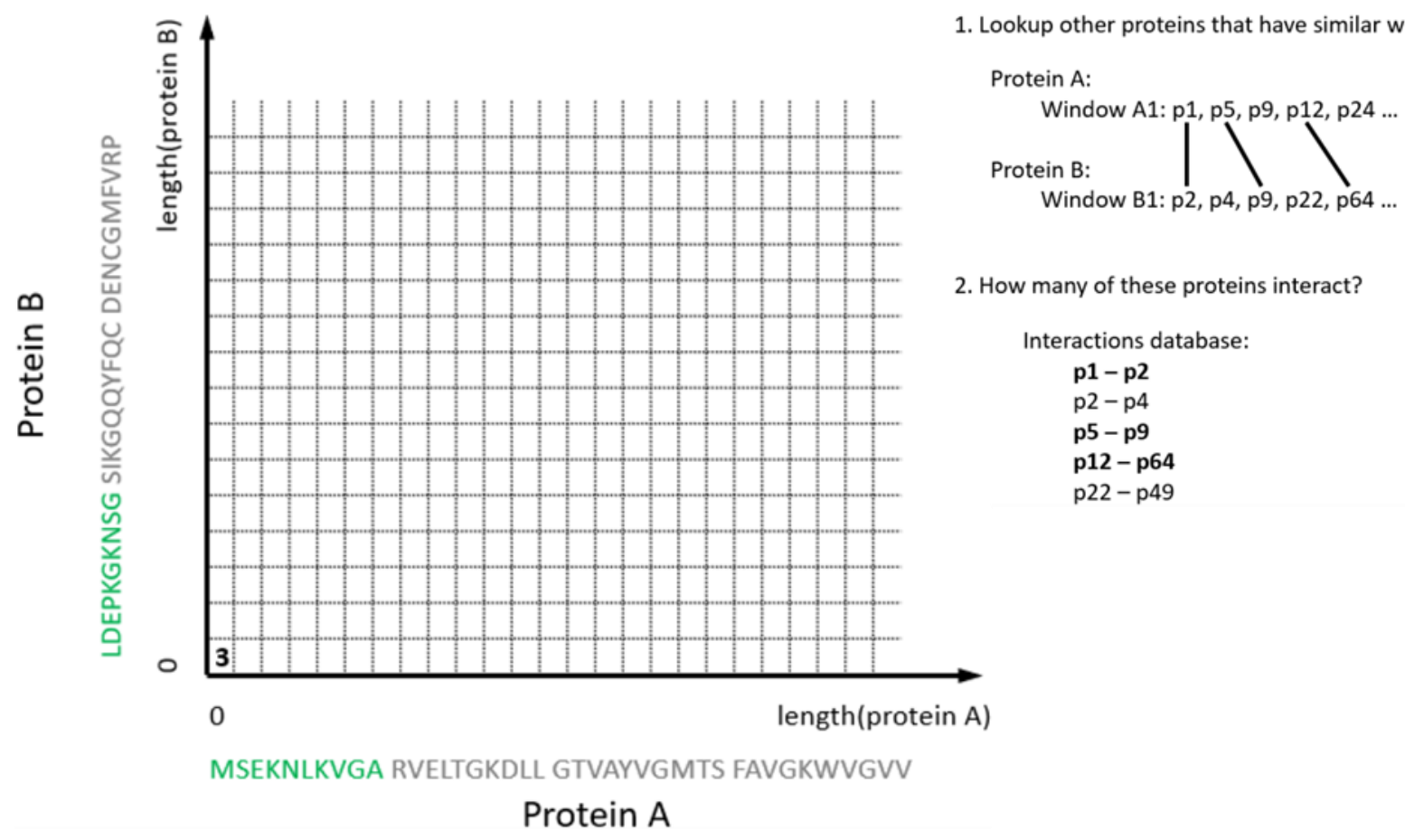

Figure 6: Overview of PIPE core algorithm - This figure examines the first sliding window of 20 amino acids in each protein, highlighted in green. In step 1, the similar proteins for the windows highlighted in green are identified. In step 2, the known interactions between the similar proteins are counted (the known interactions are highlighted in bold and drawn as black lines between proteins in the two sets). This count is entered in the interaction landscape for these windows. This process is then repeated for each pair of windows (figure reproduced from [10]) 
Iterating through the full length of both protein A and protein B leads to the creation of a 2D matrix that is as wide as the amino acid length of protein A minus nineteen, and as tall as the amino acid length of protein B minus nineteen [13]. Nineteen is subtracted due to the first twenty amino acids in the window being summarized by the first element. Each element thereafter summarizes an increment in the sliding window of one amino acid. The numerical value of each element contains the total number of similar interactions found in the provided database, and this 2D matrix with scores, illustrated in Figure 6, can be represented as a 3D landscape, presented in Figure 7.
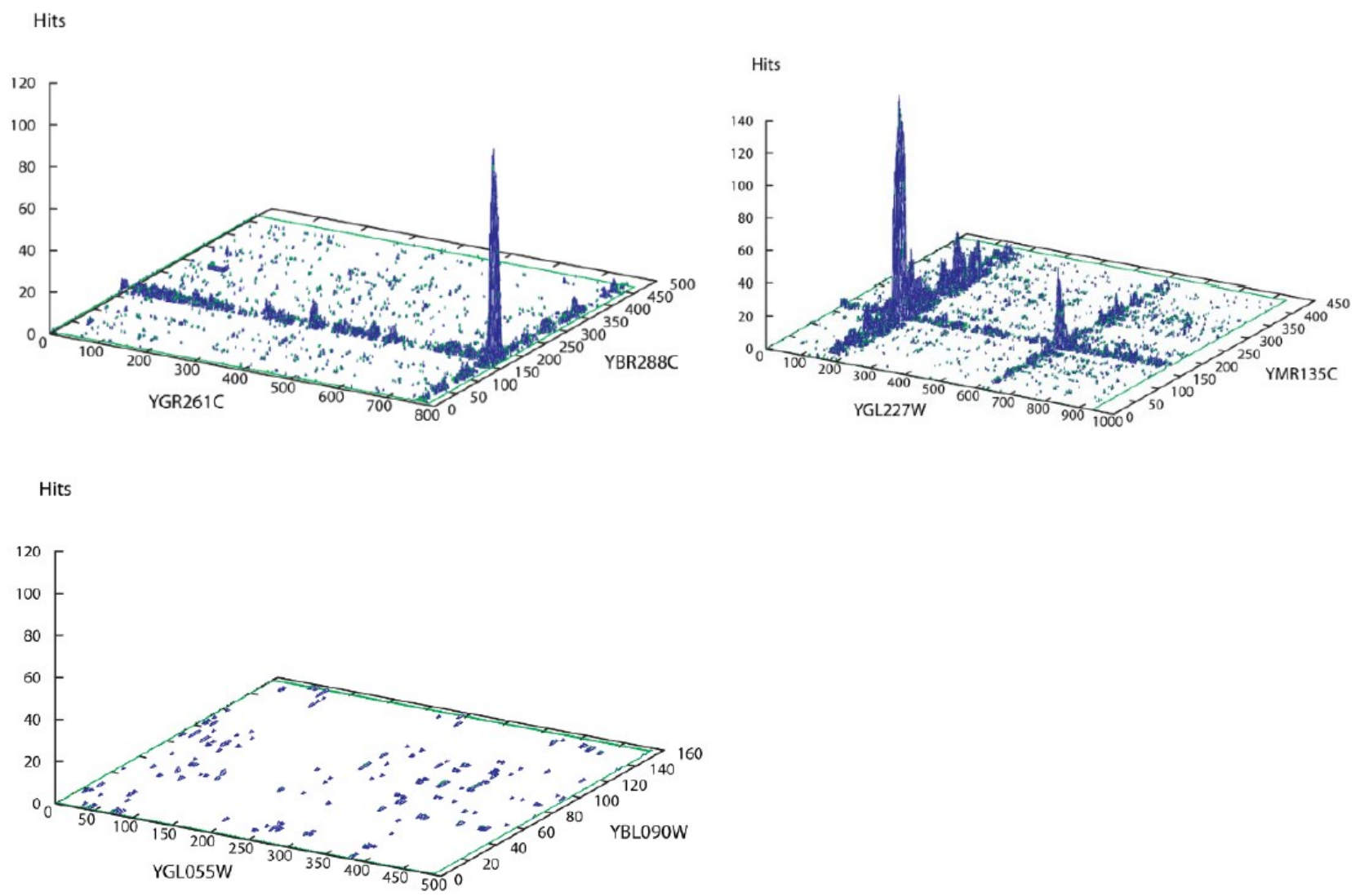

Figure 7: Sample PIPE landscapes for two positive pairs (top row) and a negative pair (bottom row) (figure reproduced from [10]) 


\subsubsection{Output Data}

The $\mathrm{x}$ and $\mathrm{y}$ axis represent the amino acids regions of the target proteins, starting from the $\mathrm{N}$-terminal amino acid at position 1 . Therefore position 5 corresponds to the 20 amino acid window starting at the fifth amino acid of the polypeptide. The score on the $\mathrm{z}$ axis represents the number of times that a pair of 20 amino acid sequences co-occurs in the dataset of interacting proteins. A high score corresponds to a high incidence of cooccurrence of the sequences among the database of interacting proteins. Therefore, a score of 5 indicates that the corresponding sequences co-occur five times in our database, whereas a score of 50 indicates that the co-occurrence is present in 50 pairs of interacting proteins. We assume that a high score represents a soaring affinity for an interaction. We note that a major source of false positives reported by PIPE is motifs with frequent occurrence in the database. Pairs of such motifs can have a high co-occurrence simply because they are very frequent [10].

The landscapes here are illustrated in 3D form, where height on the z-axis is equal the number of 'hits' or known interactions between sets of similar proteins. The height of a peak at location $(i, j)$ represents to weight of evidence that subsequences $W A_{\mathrm{i}}$ and $W B_{\mathrm{j}}$ support a physical interaction between proteins A and B. By aggregating the height of all locations in the landscape, it arrives at a total score for the putative interaction between proteins A-B [13]. It is also possible to estimate the site of interaction within each protein by examining the landscape itself, which is discussed in the coming sections, known as PIPE-Sites. Since these landscapes become input to our site detection methods, changing 
the sliding window size from 20 may have an impact on input landscape, thus impacting our methods. However, it is outside the scope of this work.

\subsubsection{SW-Score and SW-landscape}

After generating the full landscapes of PPI for a protein pair, the landscape needs to be summarized into a single value for further classification. It is done in two different ways. The first method, termed as 'traditional (or original) PIPE score', that involves a type of filter to the landscape. It includes by setting a matrix element to a value of one, if the element and its eight neighbouring entries are all non-zero, otherwise it sets it to zero. The final score is calculated as the average of the modified landscape.

The second method of summarizing the landscape into a single score is known as the similarity-weighted (SW) method [13]. This method was developed because certain sliding windows are seen in a very large number of proteins but are not responsible for supporting or mediating interactions. For example, frequently observed subsequences may have other non-PPI functions, such as sub-cellular localization signaling. The SW method normalizes the height of the landscape at a point by counting how many possible interactions there would be if every pair of similar proteins interacted. For location $(i, j)$ this normalization factor would be $\left|\operatorname{simprots}\left(W A_{\mathrm{i}}\right)\right|^{*}\left|\operatorname{simprots}\left(W B_{\mathrm{j}}\right)\right|$, where simprot is an array holding the number of proteins it interacts at each index. For example, $\operatorname{simprots}\left(W A_{\mathrm{i}}\right)$ equals to number of proteins that protein- $A$ interacts at its $i^{\text {th }}$ location. This removes the effect of windows that are very common but do not cause interactions and strengthens the effect of windows that are relatively rare but are frequently seen in known interactions. This method is 
illustrated in Figure 8 for a single pair of windows. After the score for each pair of windows is divided by the normalization factor, the overall SW score is simply the average value of the landscape. The SW method was developed in [48] and compared to the original method; overall the SW method was shown to be superior to the original method at all operating points, and is the main score used by PIPE.

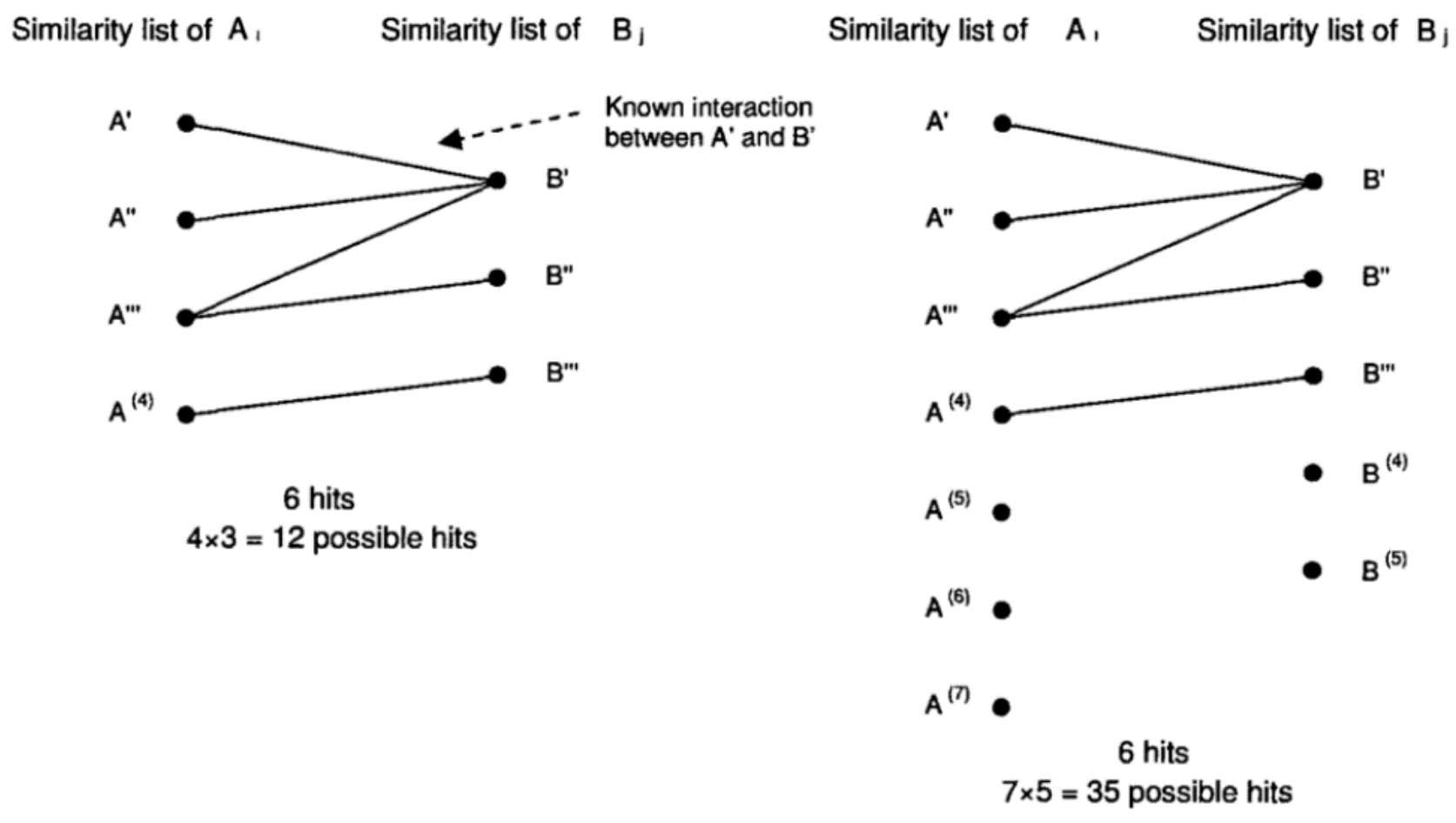

Original score: 6

Accounting for
uniqueness:
Original score: 6

$\begin{aligned} & \text { Accounting for } \\ & \text { uniqueness: }\end{aligned} \quad \frac{6}{35}=0.17$

\section{(a)}

(b)

Figure 8: Similarity Weighted (SW) method for modifying PIPE original-landscapes (figure reproduced from [13]) 


\subsection{Computational PPI Interaction Site Prediction}

PPI prediction methods attempt to determine if a pair of proteins will physically interact; this is a yes/no binary question. For a pair of interacting proteins, additional insight can be gained by determining the specific protein subsequence that is responsible for the interaction. As mentioned above, some methods seek to identify the PPI interface, defined as those amino acids that are in contact ( $>2$ angstroms) with amino acids from the other protein [49]. This leads to a potentially non-contiguous region since protein structure can bring sequence-distant amino acids into close 3D proximity. In this thesis, we instead define the PPI interaction site as the entire protein subsequence that is required to support the PPI. Some amino acids may be required to provide scaffolding to form a binding pocket; these residues may be far from the actual interface but are still critical to supporting the PPI. In this thesis, as with previous research, the PPI site is a contiguous region of each protein [7].

\subsubsection{Interaction Site Prediction}

Several computational methods have been developed to predict interaction sites. The input to these methods is typically a protein pair, while the output is the subsequence on each protein that is considered to form the interaction site. One of the earliest sequence-based PPI site prediction method is PIPE-Sites, developed at Carleton University [7]. This method is described in detail in the further section. Here we discuss other PPI prediction methods. 


\subsubsection{Xueli}

A sequence-based PPI prediction method is proposed by XueLi in [50] . It aims to predict the likelihood of interaction of the input protein pair using the physiochemical properties of the constituent domains. It uses a Support Vector Machine model for training on a database of known domain-domain interaction data [51] and the physiochemical properties of each domain. It showed promising results for predicting the likelihood of a PPI, but unable to predict the specific binding site (a.k.a PPI sites)

\subsubsection{InSite}

Where XeuLi targets to just predict PPI, InSite is a method for predicting PPI sites on a proteome-wide scale. However, InSite only aims to recognize a motif on one side of the interaction [52]. It takes as input a library of preserved sequenced motifs, a heterogeneous data of index of protein-protein interactions, obtained from different assays, and any accessible additional proof of protein-protein interactions and motif-motif interactions, like expression correlations, Gene Ontology annotations, and domain fusion.

\subsubsection{SCRIBER}

SCRIBER [53] is another sequence-based protein binding residue predictor for a given input protein sequence. It uses the following features: putative relative solvent accessibility, evolutionary conservation; relative amino acid propensity for binding; and several relevant physiochemical properties including putative protein binding intrinsically disordered regions, putative secondary structure, and selected physicochemical properties 
of amino acids (aliphaticity, aromaticity, acidity, and size). The core of SCRIBER is a naive logistic regression model, where the first layer predicts which amino acid residues bind to either proteins, DNA, RNA, or small molecules. The second layer refines the predictions to only select those involved in PPI. It uses the total number of correctly predicted binding residues (PBRs) in a protein sequence as an accuracy criterion for evaluation. This method attempts to predict which residues are in contact with the binding partner, which is a different problem than we are approaching here. As described above, in this thesis we attempt to identify the entire domain that supports the PPI, not only those residues that make up the PPI interface. Also, SCRIBER attempts to identify all PBR in a single protein, rather than analyzing a pair of proteins. It is possible that a single protein has two different PPI binding sites that support PPI with different protein partners.

\subsubsection{DeepPPISP}

DeepPPISP [54] is a protein binding predicter that utilizes secondary structure of the input protein. Unlike SCRIBER, it is a deep learning-based method. Its architecture consists of a TextCNN component (a variation of convolutional layers) to extract features and two fully connected layers to classify PBR. DeepPPISP highlights its usage of both local and global information of the input protein sequence. In the input protein sequence, for each given amino acid, the local information is the neighboring seven amino acids, and the global information is its neighboring five-hundred amino acids. DeepPPISP empirically shows that the usage of global information largely helps in the prediction. It also uses total number of correctly predicted binding sites in a protein sequence as accuracy criteria for its evaluation. Like SCRIBER, this method attempts to identify individual PBR, rather than the 
entire PPI binding site. Furthermore, DeepPPISP only examines single proteins, rather than pairs of interacting proteins.

\subsubsection{DLPred}

DLPred [55] is another sequence-based protein binding predictor. Like DeepPPISP it is also a deep learning-based method. Its architecture includes simplified LSTM layers and completely connected layers as the key components. The authors created a simplified LSTM with the primary goal of reducing time consumption. Position specific scoring matrix (PSSM) data, physical properties, hydropathy index, physicochemical characteristics, PKx (the negative logarithm of the dissociation constant for every other group in the molecule), conservation ranking, and one-hot encoding of protein sequences are the features used for training DLPred. This method adopts a filtered sampling technique such that sequences with more binding residue are selected during training. This approach helps deal with the class imbalance of the protein binding prediction problem. As with the methods above, only a single protein sequence is analyzed, rather than identifying the PPI interaction site for a specific interacting pair of proteins.

\subsubsection{DELPHI}

The latest sequence-based interaction site prediction method is DELPHI (Deep Learning Prediction of Highly Probable Protein Interaction Sites) [56], which is a sequence-based deep learning method for predicting interaction sites in a single protein (i.e., not a pair). DELPHI utilizes existing and novel AA characteristics to predict the interaction sites for an input protein sequence. Its architecture involves combining CNN and RNN (Recurrent Neural Network) for the purpose of ensemble learning. The training of the ensemble model 
is done with 11,063 protein sequences obtained from Uniprot [57]. Training and prediction use more than 10 features that are focused on AA in the protein sequence. Therefore, based on the properties of AA and existing knowledge of their roles in interactions, DELPHI is able to predict the interaction sites for the input protein sequence for its possible proteinprotein interactions.

By predicting binding sites from domain motifs, some sequence-based methods [58], [59], [60], are able to overcome some limitations of structure-based prediction. Since consensus motifs must be manually curated, and training sets in the form of high-quality binding site location databases are scarce and expensive to generate, model training is limited in each case. Furthermore, methods that depend solely on domain-motif binding are unable to establish which domain and/or motif is responsible for binding when either protein in the interacting pair has multiple domains or motifs.

The validation of predicted sites is likewise an area that can be improved. The quality and quantity of lab-confirmed interaction sites should be improved to give more exact evaluation of PPI interaction site prediction methods. Expanding the amount of available validation data will add statistical significance to predicted outcomes.

\subsubsection{PIPE-Sites}

As with Section 2.3.2, we here provide a detailed description of the PIPE-Sites method of interaction site prediction, since the contributions in this thesis build upon this method. PIPE-Sites attempts to identify peaks in the PIPE-Score landscapes produced by PIPE to 
predict protein binding/interaction sites. To check PIPE-Sites' predictions, there is a way to make use of an experimentally affirmed approval set of protein pairs and the mediating sites on every protein. We assess our predictions quantitatively by estimating the distance between the anticipated binding site ranges and the experimental binding ranges. At last, PIPE-sites apply this technique to two sets of novel protein interactions with obscure sites and provide information about the outcomes. PIPE-Sites seems, by all accounts, to be profoundly exact concerning the experimental approved dataset and is the main such technique working at proteome scale from binary interaction data alone.

\subsubsection{Walk Algorithm Explanation}

The walk algorithm attempts to identify peaks in a PIPE-score landscape since peaks suggest the location of an interaction site [7]. The walk algorithm starts at the global maximum of the landscape and "walks" in all four directions (up, down, left, right) until the height of the peak is reduced to some percentage of the maximum height. In this way, we can algorithmically determine the cross-sectional area of the peak, and as a result, the size of the binding site. The walk proceeds in the four axial directions (up, down, left, right) until the height of the landscape drops below a given threshold. Since the peak height is highly variable, the threshold is expressed as a percentage of the peak maximum height (percentPeak) and the threshold is a tunable/learnable parameter. Lower threshold values

lead to larger predicted sites as illustrated in Figure 9. In this way, the percentPeak parameter controls the precision-recall tradeoff of the method, where lower values maximize recall at the expense of reduced precision. 


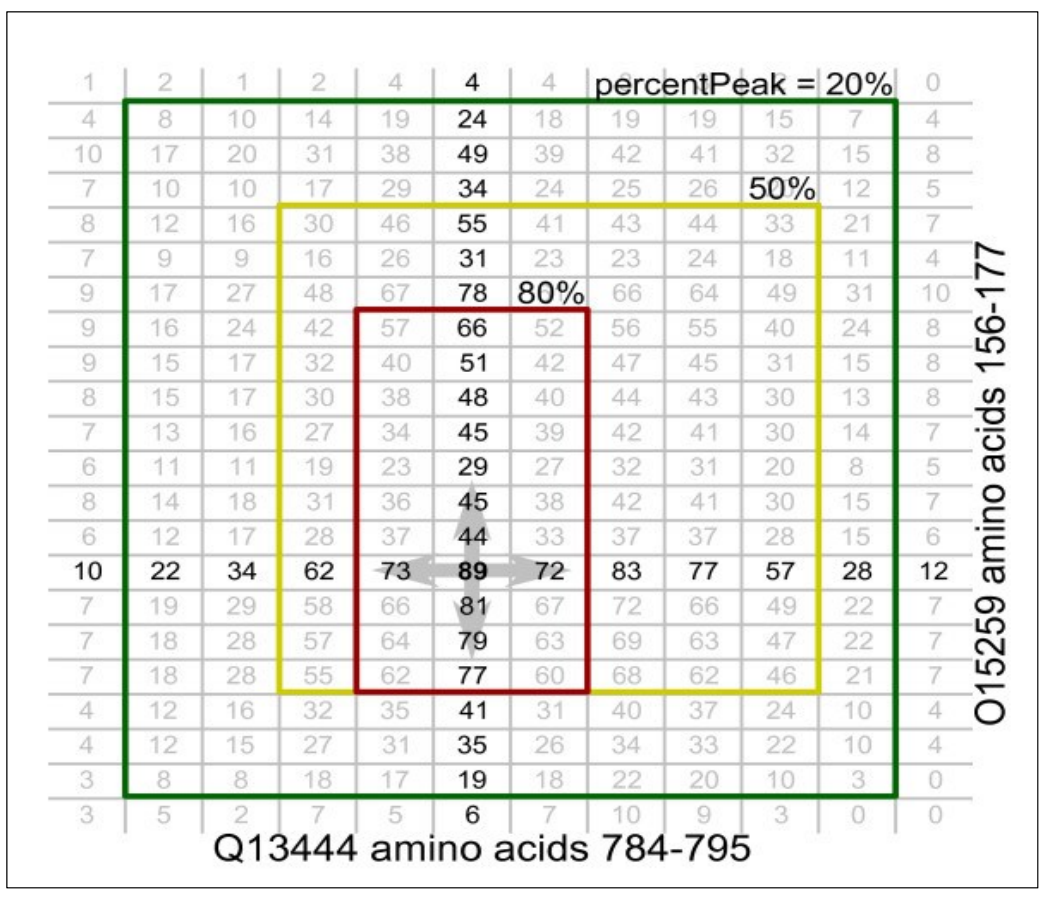

Figure 9: Walk Algorithm (figure reproduced from [7])

\subsubsection{Validation of Prediction PPI-Sites: Distance Measure}

To validate the predicted interaction sites, a comparison is made with experimentally validated interaction sites. Distance measure (DM) was introduced to compare the predicted and lab-verified (experimentally confirmed) protein interaction sites which attempts to quantify the overlap of sites on both proteins and combines these into a single value that ranges between 0 and 1 , in which 0 indicates an ideal match and 1 demonstrates that the rectangles cover opposite ends of the proteins without an overlap. For two interacting proteins $\mathrm{A}$ and $\mathrm{B}$ of length protein $\mathrm{A}_{\text {length }}$ and protein $\mathrm{B}_{\text {length, }}$ respectively, the variables startAps, endAPs, startBps, and endBps denote the location of the interaction sites predicted by PIPE-Sites while startAlab, endAlab, startBlab, endBlab denote the location of the actual/lab (experimentally confirmed) interaction sites. 


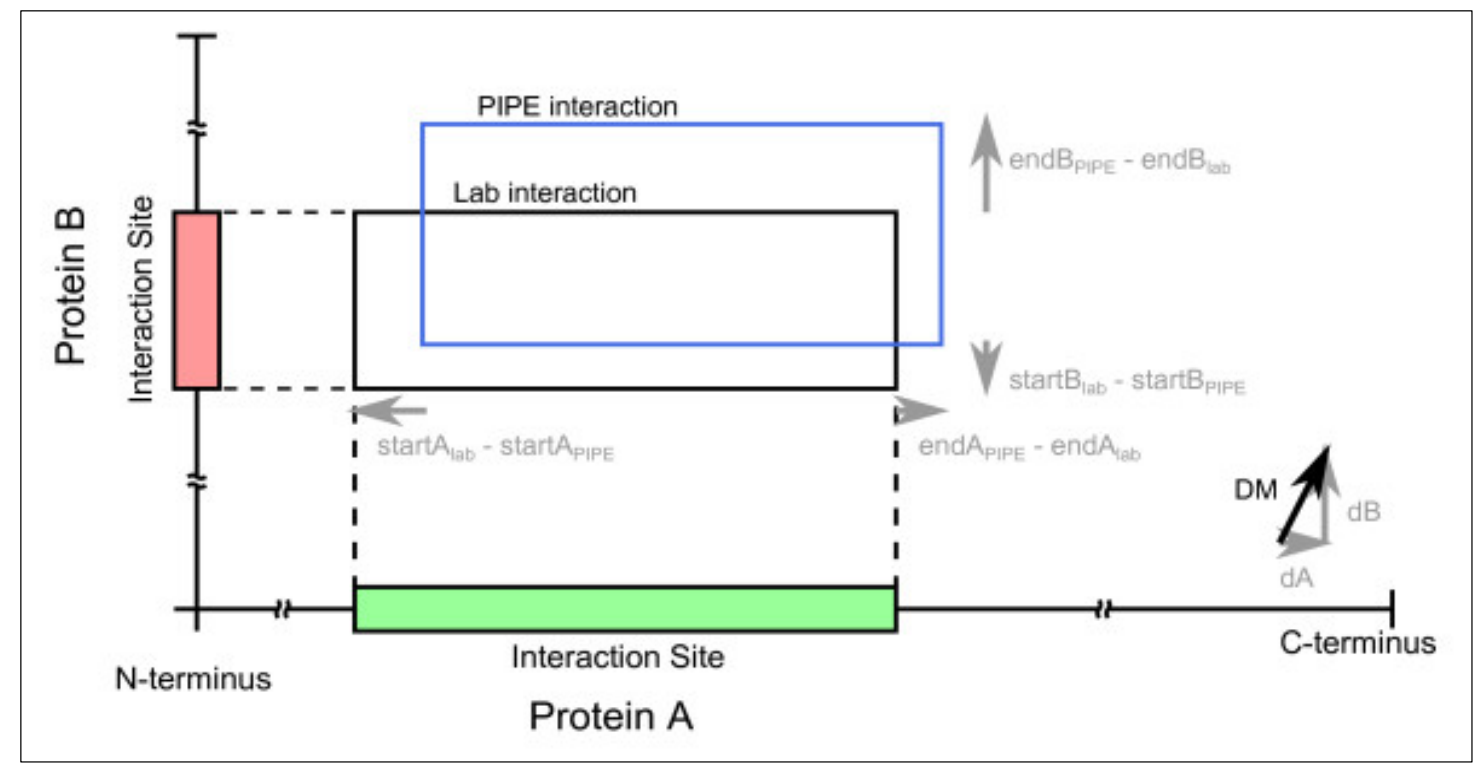

Figure 10: Distance Measure (DM) estimation (figure reproduced from [7])

It would first compute distances along both proteins by comparing the beginning positions (start $A_{\text {lab }}$ - startAps) and end positions (endA $A_{\text {lab }}$ - endAPS) and taking the maximum difference. At the point when the predicted site is contained wholly inside the lab site, the distances are both negative and we clamp the distance at 0 (ideal match) along that protein (Equation 1). This clamping means that under-prediction of interaction site is not penalized. This reflects the assumption that lab-validated sites may be larger than the true interaction site, due to the experimental (deletion) methods used to determine those sites.

The distance along each protein $(\Delta \mathrm{A}, \Delta \mathrm{B})$ are combined using Euclidean distance to form the final DM value as calculated by Equation 1 . The factor $\sqrt{2}$ guarantees that the final DM is in the range of $[0,1]$. The DM tends to favor smaller prediction sites since it does not penalize under-prediction. 


$$
\begin{gathered}
\Delta A=\frac{\max \left\{s \operatorname{start} A_{l a b}-s t a r t A_{P S}, \text { end } A_{P S}-e n d A_{l a b}, 0\right\}}{p r o t e i n A_{\text {length }}} \\
\Delta B=\frac{\max \left\{s \operatorname{start} B_{l a b}-s t a r t B_{P S}, \text { end } B_{P S}-e n d B_{l a b} 0\right\}}{p r o t e i n B_{\text {length }}} \\
D M=\frac{\sqrt{\Delta A^{2}+\Delta B^{2}}}{\sqrt{2}}
\end{gathered}
$$

Equation 1 : Distance Measure formula (reproduced from [7])

Like PIPE-Sites, the interaction site determination techniques in this thesis do not require protein structure information and can be applied to all protein pairs in a proteome.

\subsubsection{Box-Plot distribution}

A boxplot distribution is a standardized way of displaying the distribution of a variable or data based on a five-number summary ("minimum", first quartile (Q1), median, third quartile (Q3), and "maximum") [61] as illustrated in Figure 11. It also talks about the outliers in data. It can also tell if the data is symmetrical, how tightly the input data is grouped, and if and how this data is skewed. These are the values of numbers that it uses -

1. median (Q2/50th Percentile): the middle value of the dataset.

2. first quartile (Q1/25th Percentile): the middle number between the smallest number (not the "minimum") and the median of the dataset.

3. third quartile (Q3/75th Percentile): the middle value between the median and the highest value (not the "maximum") of the dataset.

4. interquartile range (IQR): 25 th to the 75 th percentile.

5. "maximum": $\mathrm{Q} 3+1.5 * \mathrm{IQR}$

6. "minimum": Q1 -1.5*IQR 


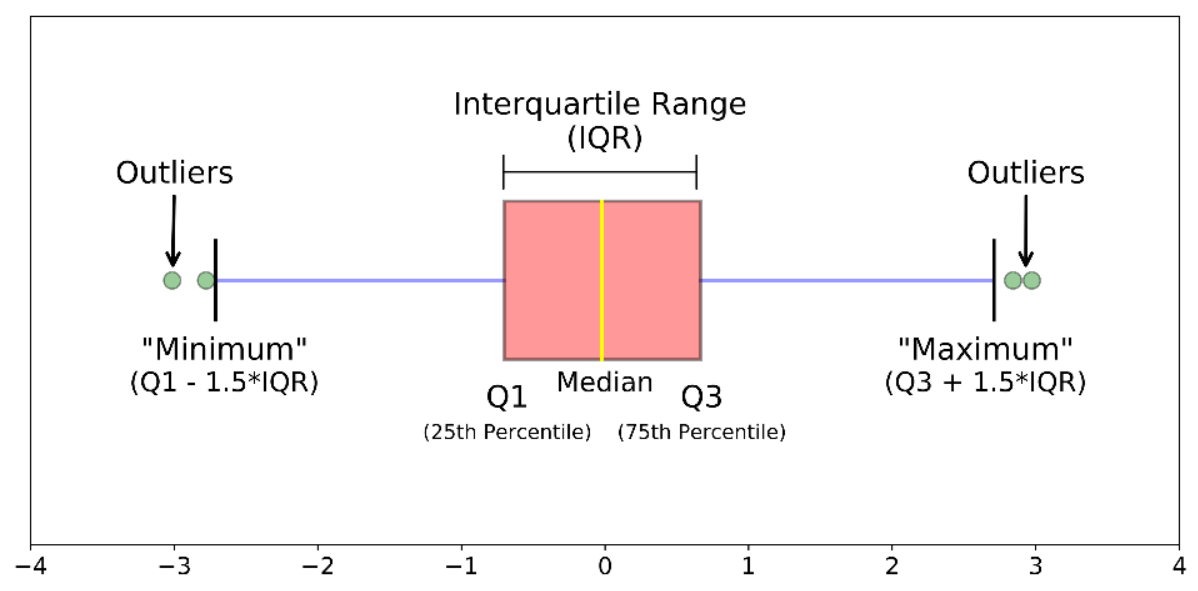

Figure 11 : Box plot as a five-number summary (figure reproduced from [61])

Using the above five number, Figure 12 illustrates a box plot for a normal distribution.

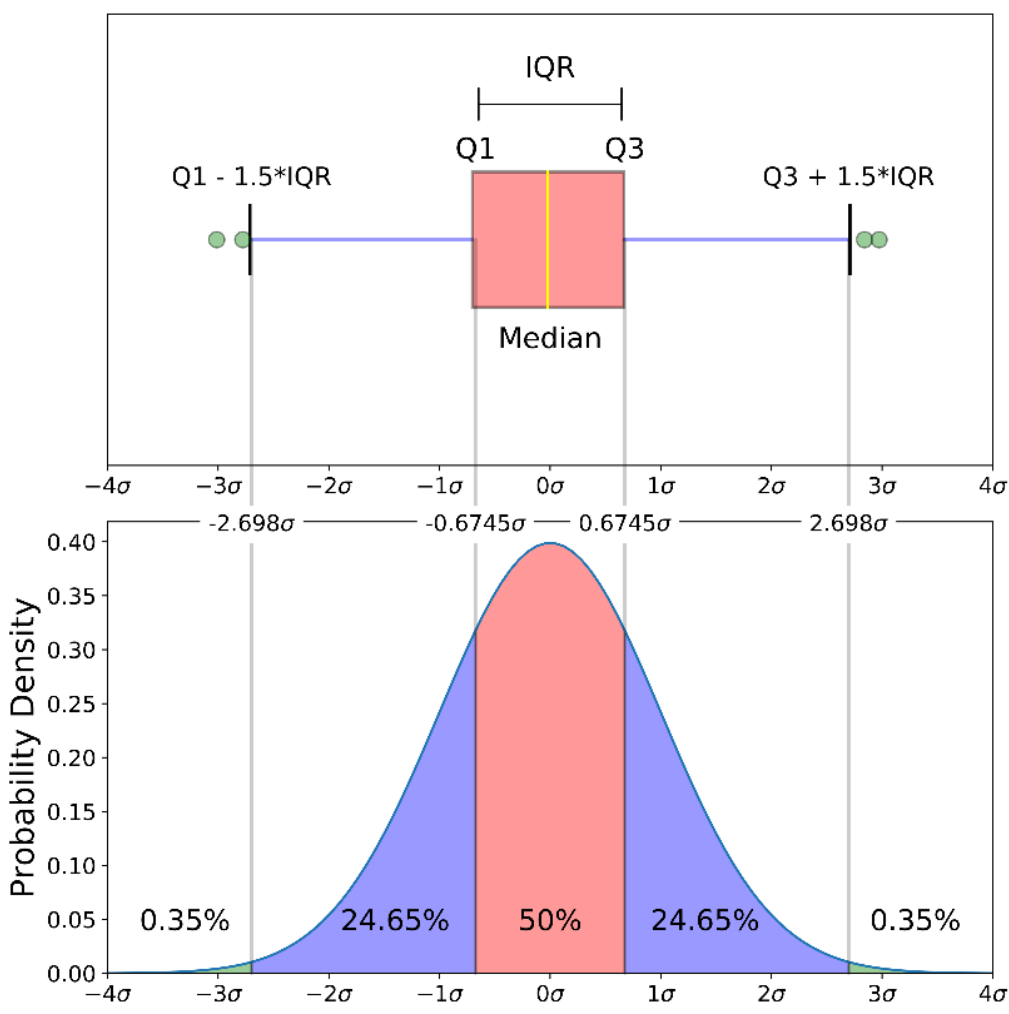

Figure 12 : Box plot for a Normal distribution (figure reproduced from [61])

Later in this work, we draw various box-plot distributions of the performance metrics, to understand the performance of different algorithms, and compare those with each other. 


\section{Assessing and Retuning PIPE-Sites}

\subsection{Introduction}

This chapter details the process of extracting new high-quality datasets of gold-standard known PPI sites. It also presents the limitations of existing performance metric originally used with PIPE-Sites and introduces an improved alternative. Using the new data and performance metrics, the original PIPE-Sites is re-tuned. Lastly, it is demonstrated that, even with retuning, the original PIPE-Sites method is incapable of interpreting SW-Score landscapes, motivating the introduction of new methods in Chapter 4.

\subsection{New High-Quality Dataset}

The most recent list of human protein pairs known to physically interact was extracted from BioGrid [62]. Here, we only considered physical interactions, of which there were 66,084 interacting protein pairs. For each protein pairs, UniProt [57] is used to get the sequences for the individual proteins. A total of 20,236 human protein sequences are available in UniProt. These two data sources are needed as the input to run the PIPE in order to obtain SW-landscapes for any protein pair. PIPE was used to generate SWlandscapes for each protein pair. The landscapes are used as input to PIPE-Sites and to the new methods introduced in Chapter 4 to predict the PPI sites.

To evaluate the accuracies of these prediction-sites, we require lab verified interactionsites locations in these interacting protein pairs. Here, we used the database of 3D Interaction Domains a collection of domain-domain interactions in proteins from various 
organisms [63]. After processing the custom file provided by database of 3D Interaction Domains (3did) [63], we obtained 14,278 pairs of domain-domain interactions.

To locate known interacting domain pairs within our interacting proteins, the Pfam server was used [64]. Pfam's REST-API was used to retrieve domain details for each protein, such as how many domains are in a protein, their sequence boundaries, etc. Use of the Pfam API resulted in domain data of each of the 20,236 protein sequences. Combining 3 did and Pfam data, we were able to categorise the available 66,084 interacting protein-pairs into three categories:

1. No-domain interaction-pairs (58,231 pairs). For these interacting protein pairs, no interacting domains could be identified.

2. Single-domain interaction-pairs (2,532 pairs). Each Single-domain interaction-pair had one domain in each of the protein that was known to interact.

3. Multi-domain interaction-pairs (5,321 pairs). Multi-domain interaction-pairs means that the interacting protein-pairs had multiple domains in at least one of the two proteins of the interacting protein-pair.

If a protein pair is known to interact and that protein pair contains a pair of domains that are specifically known to interact, then we can assume that the PPI site matches the domain boundaries in the two proteins. Therefore, all experiments of this research focused only on Single-domain interaction-pairs. These are referred to as the lab-verified sites (a.k.a. ground truth PPI site dataset). Figure 13 contains the details of this high-quality data extraction pipeline. 


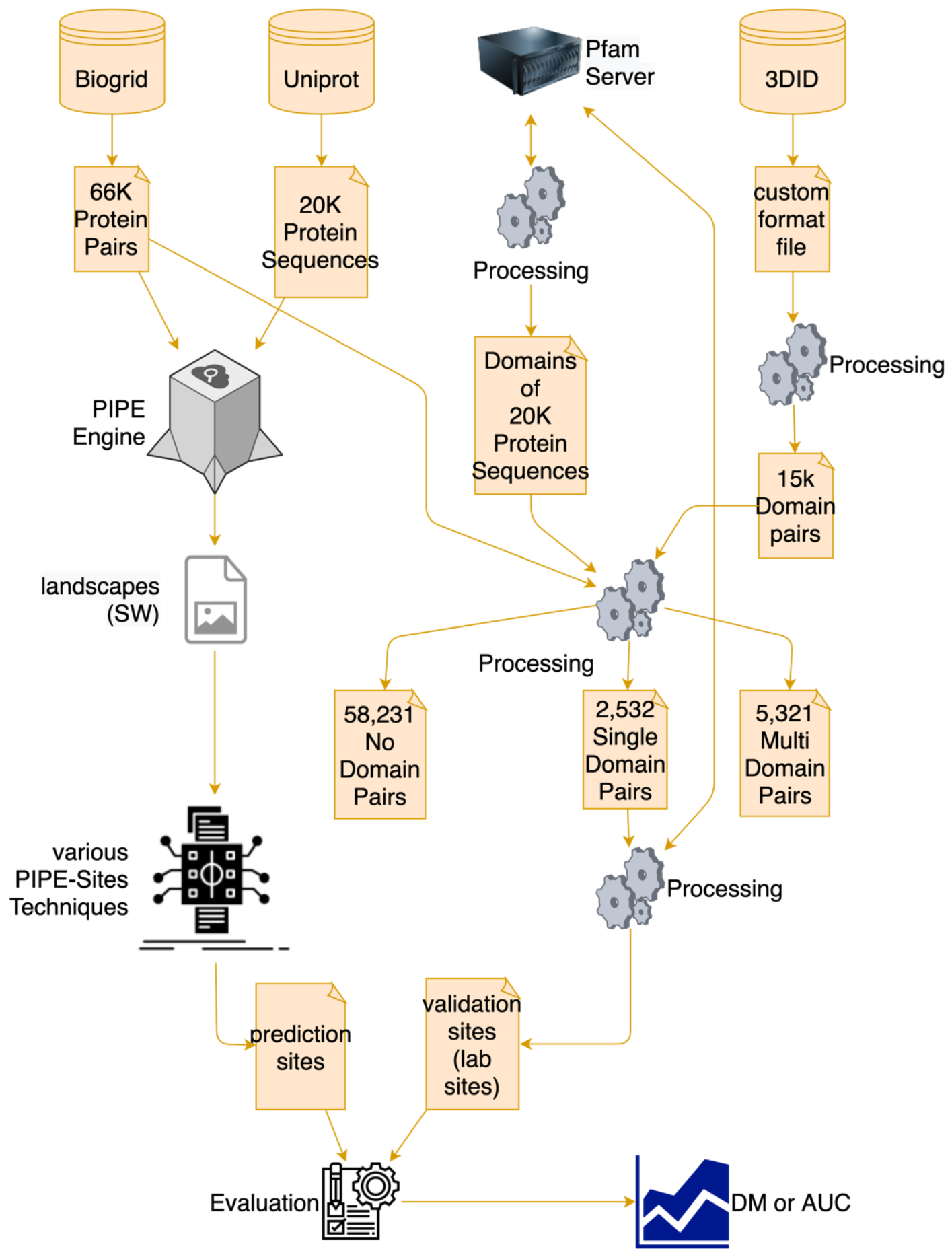

Figure 13 : New High-Quality Dataset Extraction Pipeline - A pipeline describing the process of data extraction of lab-verified domain-domain interaction sites, and how that is used to perform validations on various (existing and new) PIPE-Sites detection techniques 
This pipeline was developed in python framework and was tested to extract both human and yeast protein pairs. The framework was deployed on AWS infrastructure with m5.24xlarge specifications [65], which consisted of 64 vCPUs and 256 GiB memory. Pfam server had a maximum transactions per second (tps) limit of 5 tps per host, leading to an about 100 minutes of runtime to extract the domain level information for all human and yeast protein-pairs.

All the new methods and algorithms throughout this work, are developed in python and deployed on AWS infrastructure, unless mentioned otherwise.

\subsection{New Performance Metric}

\subsubsection{Background: Existing Metric}

Recall that PIPE produces a matrix of scores (PIPE-Score), creating a so-called landscape, for the input protein-pair. The landscape is then filtered and aggregated, and if the sum surpasses a set threshold, the input protein-pair is predicted to interact. PPI prediction is suited to a binary (Yes/No) evaluation and use of performance metrics such as accuracy, sensitivity, etc. However, PIPE-Sites predicts the location of interaction (interaction-site) and hence, the measure of its accuracy is done on a continuous scale measuring "how close" or "how far" the predicted site is to the actual lab-verified site. When the original PIPE-Sites method was developed, a simple performance metric was defined: the "Distance Measure" (DM) [7]. The DM measures overlap between predicted interactionsites and lab verified interaction-sites by assigning a single value between 0 and 1 , where 0 
indicates a perfect match. We first calculate distances along each protein by subtracting the start positions (startAlab - startAps) and end positions (endAlab - endAPs) and taking the maximum. When the predicted site is contained within the lab site, the distances are both negative and we clamp the distance at 0 (perfect match) along that protein.

\subsubsection{Proposal}

In the existing DM approach, if the prediction PIPE-site is entirely contained within the lab verified PPI-site, then it does not penalize any under-prediction. Let us consider an example where lab verified interaction site is $100 \mathrm{AA}$ long for a protein sequence. Now on the same input, let's assume one PIPE-Sites prediction technique produces prediction interaction-site as just 5 amino-acids whereas, another technique predicts the interactionsite as 95 amino-acids. Making the last assumption that both the techniques have predicted the entire prediction-site within the lab verified site. Now, for such examples, the existing DM approach gives score 0 to both the techniques. This incentivizes of prediction for smaller interaction-site leads to a bias for PIPE-Sites prediction.

We here introduce a modification in the existing DM metric by taking the absolute value of the difference, instead of signed difference clamped at 0 . The new metric is referred to as Absolute Distance Measure (ADM) and is defined in Equation 2. With this new formula applied to the example discussed earlier, the new Distance Measure value is smaller for the PIPE-Sites prediction technique with 95 amino-acids in prediction-site than the one which had 5 amino-acids in prediction-site. 


$$
\begin{gathered}
\Delta A=\frac{\max \left\{\mid \text { start } A_{\text {lab }}-\operatorname{start} A_{P S}|,| \text { end } A_{P S}-e n d A_{\text {lab }} \mid\right\}}{\text { protein } A_{\text {length }}} \\
\Delta B=\frac{\max \left\{\mid \text { start } B_{\text {lab }}-\operatorname{start} B_{P S}|,| \text { end } B_{P S}-\operatorname{end} B_{\text {lab }} \mid\right\}}{\text { protein } B_{\text {length }}} \\
D M=\frac{\sqrt{\Delta A^{2}+\Delta B^{2}}}{\sqrt{2}}
\end{gathered}
$$

Equation 2 : Absolute Distance Measure - a new metric introduced

\subsubsection{Conclusion}

This new Absolute Distance Measure brings the best of both, the existing DM and the traditional JI measure. Since it does not give flat 0 value when the prediction PPI-site is inside lab verified interaction-site and measures the degree of overlap like JI does. At the same time, it does not give a flat 1 value when there is no overlap between prediction PPI-site and lab verified interaction-site, but provides a continuous value between 0 to 1 based on how far the prediction PPI-site is (like existing DM). Thus, as a metric, it provides a simultaneous measurement of overlap and distance, between predicted and actual PPI-sites. Henceforth, we used the new Absolute Distance Measure (ADM) as evaluation metric in all the experiments of this research work. It penalizes under-prediction as well as over prediction. Since ADM is a measurement where a score of 0 denotes that the prediction PPI-site and the lab verified PPI-site are exactly same, whichever PPI-site prediction method produces lower ADM scores, is considered to be a better method from the ones having high ADM scores. Henceforth, we would be looking for lower ADM score for identifying high performing methods. 


\subsection{Optimizing Inherent Parameters in PIPE-Sites}

As described with detail in Section 2.4.2, the existing PIPE-Sites method uses the WALK algorithm to predict the PPI-sites using the original-PIPE landscapes. This WALK algorithm has a few parameters such as threshold value, minimum peak height, minimum base height etc. However, the previous work [7] concluded the threshold to be the most effective hyper-parameter and therefore performed parameter tuning on it. Threshold was generally set as some percentage of the peak value, where the peak of a landscape is defined as the maximum value of that landscape at any index of the input protein-pair. The experiments conducted in the original PIPE-Sites study [7] led to the conclusion of threshold value as $50 \%$ (of the peak height) to be the best for the available dataset. This hyperparameter was tuned on a relatively small dataset, 423 human protein -pairs.

WALK algorithm inherent parameter tuning on original-landscapes

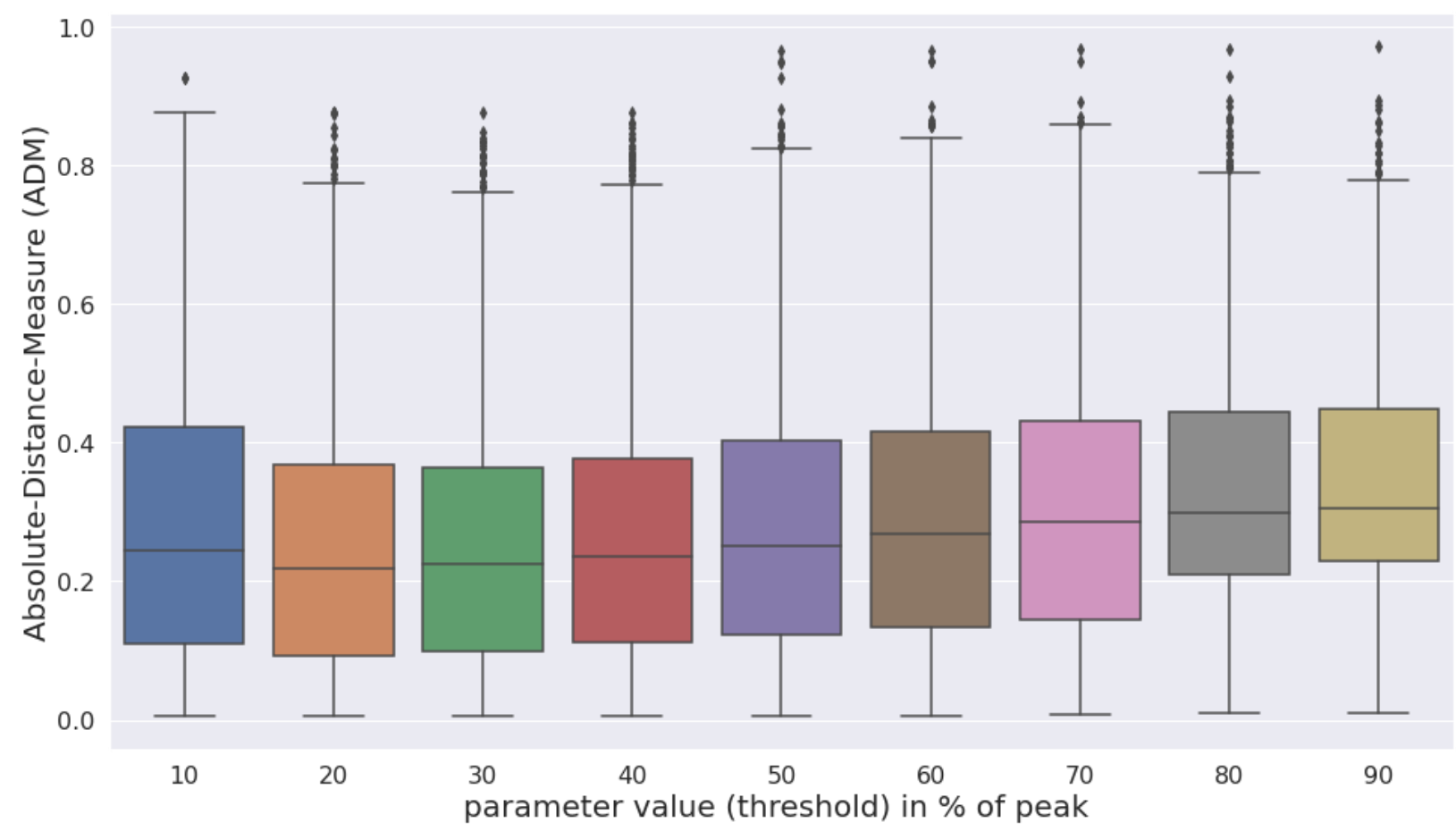

Figure 14 : Inherent parameter tuning in WALK (original method for detecting PIPE-Sites) 
After introducing the new high-quality dataset and improved performance metric (ADM), we are motivated to re-tune the threshold within the WALK. Figure 14 illustrates a box-plot distribution for ADM scores. We are using such box-plots to illustrate and explain the findings of parameter tuning throughout various algorithms. We have used Grid-Search Cross-Validation [66] [67] for parameter tuning, where we perform a 5-cross validation on all values of the parameter from the given parameter-grid. For example, in WALK, the threshold values are ranging from $10 \%$ to $90 \%$ (of the peak) with the interval of $10 \%$. We continue to use Grid-Search Cross-Validation in this manner for parameter tuning of all the other algorithms. There also, we use 5-fold for cross validation, but the values of parameter-gid, used for grid search, are discussed in the respective sections. Hence, Figure 14 illustrates a box-plot distribution of final ADM values of the best of the three PPISites for WALK algorithm on all the protein-pairs. The $\mathrm{x}$-axis represents various values of threshold (ranging from $10 \%$ to $90 \%$ of the peak) and y-axis represents the distribution of scores of ADM for all the protein-pairs. It is observed that the optimal value of threshold is reached at $30 \%$ of the peak value.

We can interpret that running WALK with low threshold values signifies that we are considering almost the entire landscape as the predicted interaction-site. Similarly running WALK with high threshold values signifies that we are considering smaller sites as we increase the threshold value. Figure 14 clearly shows that both the extremes are not the optimal values but a threshold of $30 \%$ (of the peak height) comes out to be the optimal one where ADM reaches its minimum values. Therefore, we conclude that the WALK 
algorithms, when retuned with a new high-quality dataset and measured on an improved performance metric (ADM), performs best at the threshold value of $30 \%$ (of the peak value).

Since we know that SW-landscapes produces better representation of PPI, we would like to examine the performance of WALK with it. Hence, we use SW-landscapes produced for the latest dataset, as the input in our next section to evaluate the performance of WALK.

\subsection{PIPE-Sites on SW-Landscapes}

One of the key motivations for this thesis is to find a way to leverage the SW-score, used to great effect in PIPE. As a reminder, the SW-score differs from the PIPE-score in that each element in the landscape is normalized by the frequency of occurrence of the corresponding sequence windows across the entire proteome. This will penalize frequently occurring peptides, while increasing the weight on unique peptides strictly associated with known PPI. This same insight should be effective when predicting PPI interaction-sites. As a first attempt, the Walk algorithm used in the original PIPE-Sites method is applied to SWscore landscapes.

The existing PIPE-Sites prediction technique, WALK, works best with parameter value $30 \%$ (of the peak height) when evaluated on PIPE landscapes for the latest dataset. Now we run the WALK with this parameter value on the SW-landscapes for the same latest data set. 
Comparing WALK algorithm on original-landscapes vs. SW-landscapes

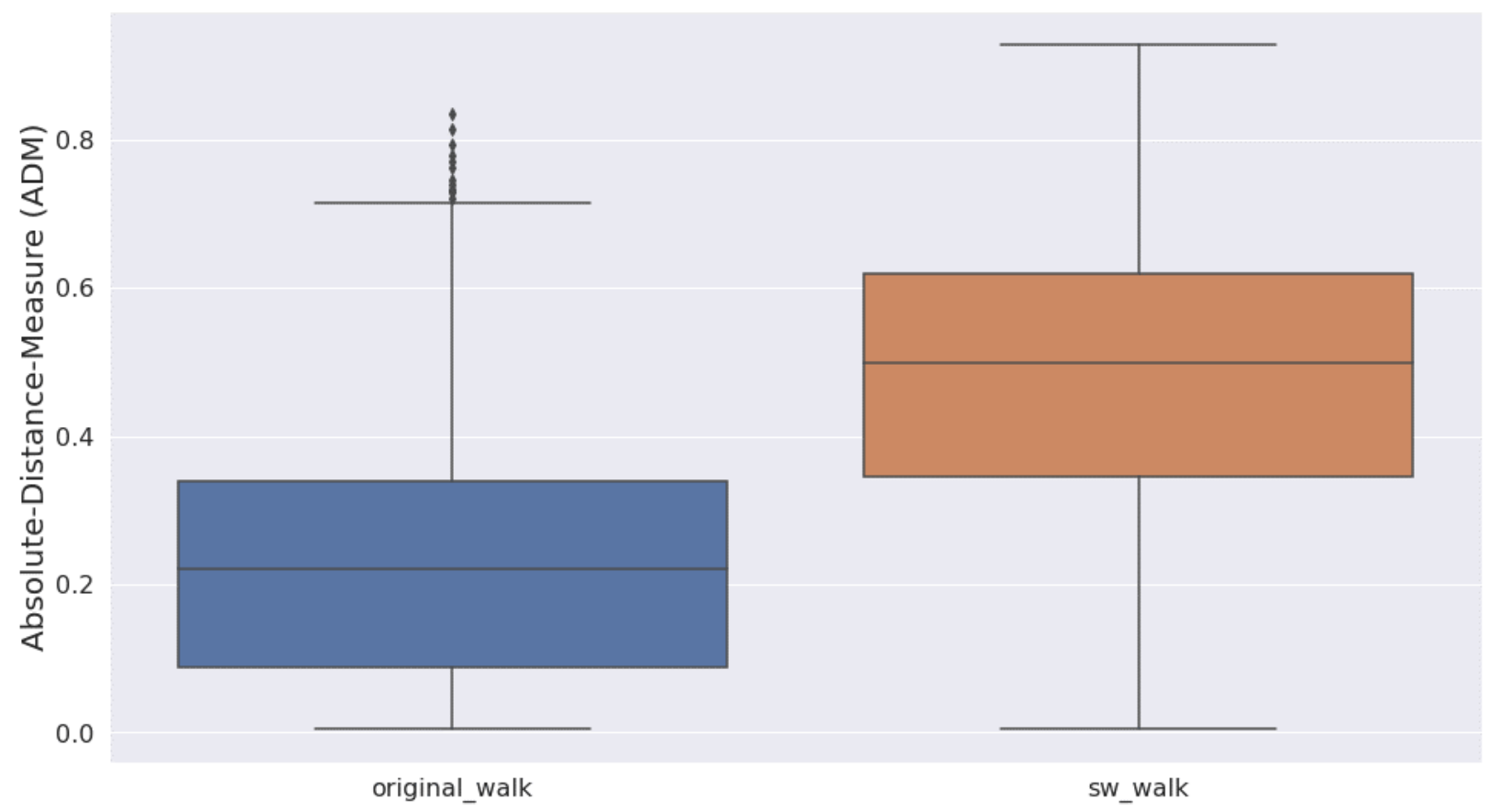

Figure 15 : Poor performance of WALK algorithm when applied to SW-landscapes (over new high-quality dataset)

Figure 15 shows that, when applied to SW-landscapes of new high-quality dataset, WALK is not performing well in predicting PPI-sites. We experimented with changing parameters of WALK but that also yielded similar results; that is, WALK performing equally poorly for all parameter choices on these SW-landscapes (as observed in the Figure 16). This motivated us to explore new techniques that should work well in predicting PPI-sites on these new SW-landscapes. In the next chapter we will discuss these new techniques in detail. 


\section{WALK algo on SW-landscapes}

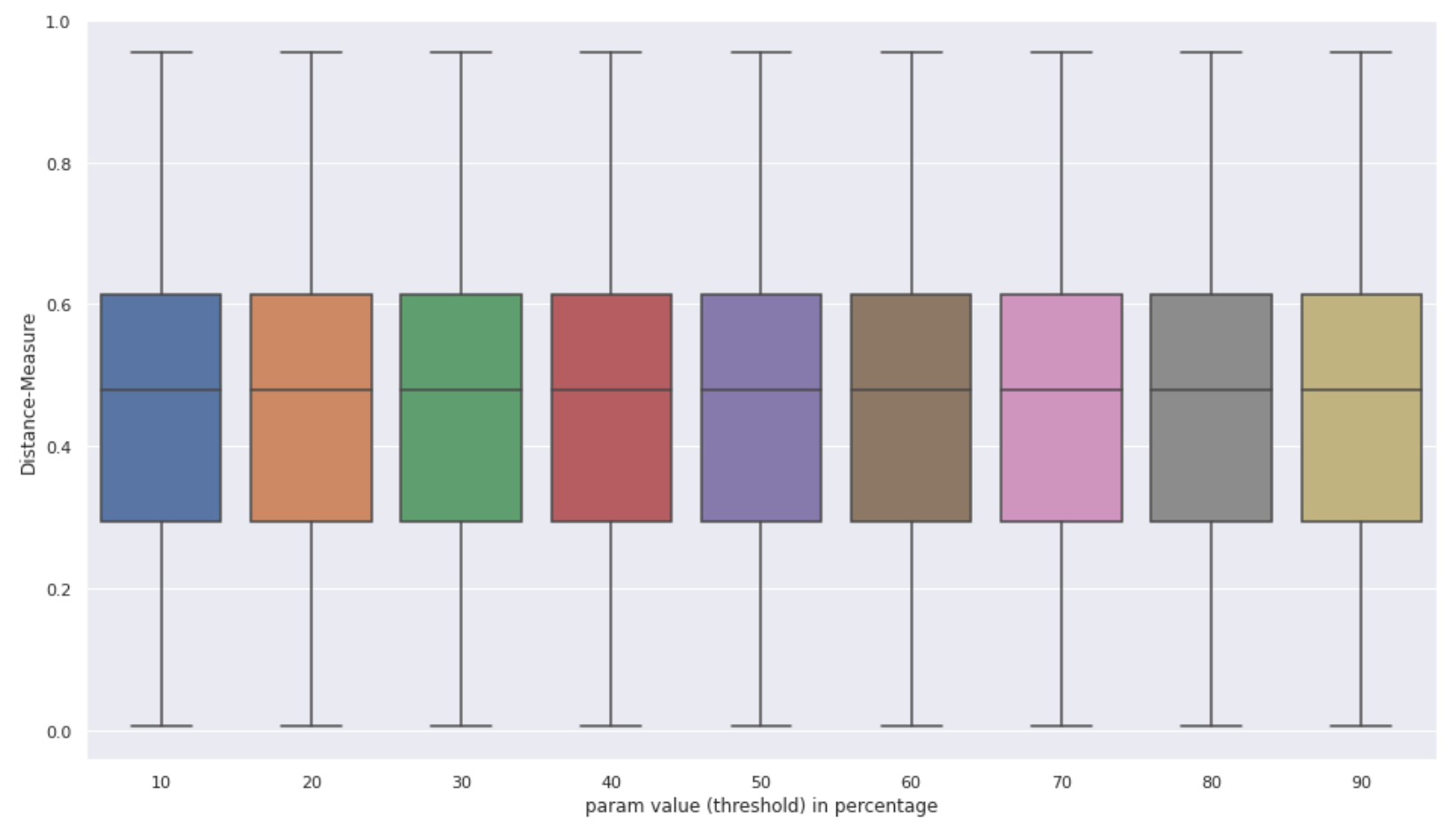

Figure 16 : SW-landscape parameter tuning: performance of WALK on SW-landscapes with Varying parameters

Both the experiments of WALK on original-landscapes and SW-landscapes were performed on AWS infrastructure with m5.24xlarge specifications [65], which consisted of 64 vCPUs and 256 GiB memory. 


\section{Introducing New PPI-Sites Detection Techniques}

\subsection{Introduction}

Our experiments in Section 3.5 concluded that the WALK algorithm used in the original PIPE-Sites method is not an effective approach to determining interaction-sites from SWlandscapes. In this chapter we introduce and evaluate three new techniques for determining interaction-sites from SW-landscape data. Each method is described and evaluated in the following sections. Additionally, we perform a holistic comparison of all these techniques to arrive at a final recommendation.

\subsection{PANORAMA - a Greedy Algorithm}

This technique is heavily influenced by the original WALK algorithm. Recall that WALK selects the peak of a landscape and starts exploring only four directions, up, down, left, and right, from here. For example, while going in left direction, it considers moving one step forward to left, only if the value to the left is above threshold; otherwise, it stops moving in left direction. It does this for all four directions. This means that WALK is a classic greedy algorithm [68], where we decide to either move forward in the direction chosen or just stop, by making only locally optimal choices at each step. And second, is that the WALK considers only scores in the same row and column as the peak value. Walk considers just one value of Protein-B at a point, to find the domain of Protein-A. This is illustrated in Figure 17, which shows a small patch of a PIPE landscape where the local peak value (100) is at location $(2,3)$. To grow the interaction site, the WALK algorithm will begin with a $1 \mathrm{x} 1$ site at $(2,3)$ and then grow the site upwards until the value in column2 drops below 91 
(assuming a threshold of 0.91 is used). Likewise, it will extend the site downwards until the value in column2 drops below 91, to the right until the value in row3 drops below 91, and to the left until the value in row3 drops below 91. However, the landscape values that fall outside row3 and column 2 are not considered by the WALK algorithm. In effect, the WALK algorithm will miss peaks that are not axis-aligned; for example, it will miss diagonal ridges.

\begin{tabular}{|c|c|c|c|c|c|c|c|c|c|c|c|}
\hline 9 & & & & & & & & & & & \\
\hline 8 & & & & & & & & & & & \\
\hline 7 & & 83.4 & 80.0 & 84.4 & 87.8 & 89.7 & 90.2 & 89.4 & 87.7 & 85.3 & \\
\hline 6 & & 86.9 & 85.0 & 88.9 & 91.1 & 91.6 & 90.7 & 88.7 & 86.0 & 82.8 & \\
\hline Protein-B & & 88.8 & 90.0 & 92.8 & 93.3 & 92.1 & 89.8 & 86.7 & 83.3 & 79.6 & \\
\hline 4 & & 87.5 & 95.0 & 95.5 & 93.8 & 90.9 & 87.4 & 83.7 & 79.9 & 75.9 & \\
\hline 3 & & 80.0 & 100.0 & 96.0 & 92.0 & 88.0 & 84.0 & 80.0 & 76.0 & 72.0 & \\
\hline 2 & & 85.0 & 90.0 & 93.0 & 92.5 & 90.3 & 87.1 & 83.6 & 79.8 & 75.9 & \\
\hline 1 & & 82.5 & 80.0 & 86.5 & 89.5 & 89.9 & 88.5 & 86.0 & 82.9 & 79.4 & \\
\hline 0 & & & & & & & & & & & \\
\hline & 0 & 1 & 2 & 3 & 4 & 5 & 6 & 7 & 8 & 9 & 10 \\
\hline & & & & & & Protein-A & & & & & \\
\hline
\end{tabular}

Figure 17 : WALK Site Detection - WALK considers only scores in the peak row and column

In our new technique, PANORAMA, we start with the same intuition of greedy approach of WALK and expand it to consider more than one amino-acid sequence while predicting the PPI-site. Meaning, we would be considering the diagonal ridges that WALK missed. This becomes our intuition behind PANORAMA. We hope considering the diagonal ridges helps in predicting the location of PPI more accurately, since it does not consider only one location of AA like the way WALK does. We describe the core algorithm for PANORAMA in the next section.

\subsubsection{Core Algorithm}

Before diving into the details of the core of the algorithm, defining few terminologies that are used later would be helpful. To begin with, a candidate PPI-site is an axis-aligned 
rectangle in a SW-landscape matrix as a square. As illustrated in Figure 18, let us consider a rectangle having $\mathrm{x}$-axis (protein-A) with indexes $2,3,4$ and $\mathrm{y}$-axis (protein-B) with indexes 3, 4. It is illustrated with dashed lines in the Figure 18. Now we define the volume of this rectangle as the sum of all the values inside this rectangle, which is $572.3=100(2,3)+$ $96(3,3)+92(4,3)+95(2,4)+95 \cdot 5(3,4)+93 \cdot 8(4,4)$.

\begin{tabular}{|c|c|c|c|c|c|c|c|c|c|c|c|}
\hline \multicolumn{12}{|l|}{9} \\
\hline \multicolumn{12}{|l|}{8} \\
\hline 7 & & 83.4 & 80.0 & 84.4 & 87.8 & 89.7 & 90.2 & 89.4 & 87.7 & 85.3 & \\
\hline 6 & & 86.9 & 85.0 & 88.9 & 91.1. & 91.6 & 90.7 & 88.7 & 86.0 & 82.8 & \\
\hline Protein-B & & 88.8 & 90.0 & 92.8 & 93.31 & 92.1 & 89.8 & 86.7 & 83.3 & 79.6 & \\
\hline 4 & & 87.5 & 95.0 & 95.5 & 93.8 & 90.9 & 87.4 & 83.7 & 79.9 & 75.9 & \\
\hline 3 & & 80.01 & 100.0 & 96.0 & 92.0 & 88.0 & 84.0 & 80.0 & 76.0 & 72.0 & \\
\hline 2 & & 85.0 & 90.0 & 93.0 & 92.5 & 90.3 & 87.1 & 83.6 & 79.8 & 75.9 & \\
\hline 1 & & 82.5 & 80.0 & 86.5 & 89.5 & 89.9 & 88.5 & 86.0 & 82.9 & 79.4 & \\
\hline \multicolumn{12}{|l|}{0} \\
\hline & 0 & 1 & 2 & 3 & 4 & 5 & 6 & 7 & 8 & 9 & 10 \\
\hline & & & & & & Protein-A & & & & & \\
\hline
\end{tabular}

Figure 18 : Illustrating definitions of upper-bar, lower-bar, right-bar and left-bar

Second, for every rectangle, we also define a value called maximum possible volume. This value is equal to volume of a hypothetical rectangle of the same dimension, but each index of that hypothetical rectangle has the peak value. Meaning that the maximum possible volume of the rectangle with the same above example would be 600 , given the peak value is 100. Pseudo code for this function is described in Figure 19 from line number 00 to 01 . Third, we define the expansion of the rectangle. Any rectangle has four choices to expand: upper-bar, lower-bar, right-bar, and left-bar. Upper-bar of the rectangle is defined as the rectangular bar on top side of the rectangle. This upper-bar is obtained by adding just one value to the maximum value of rectangle's y-axis. For example, upper-bar for the rectangle used in the above example would be a rectangular bar containing indexes $(2,5),(3,5)$ and $(4,5)$. Similarly, the lower-bar would be a rectangular bar containing indexes $(2,2),(3,2)$ and $(4,2)$, which is obtained by subtracting one value from the minimum value of 
rectangle's y-axis. The right-bar would be a rectangular bar containing indexes $(5,3),(5,4)$ and lastly, left-bar would be a rectangular bar containing indexes $(1,3),(1,4)$. Figure 18 is illustrating this example with all these four bars marked around the rectangle. Once we define these bars, for a rectangle at any time during execution of our algorithm, we calculate the average volume of all four bars. Note that we calculate average volume of the bar and not the total volume. Pseudo code to calculate the average volumes of all these bars is described in Figure 19.

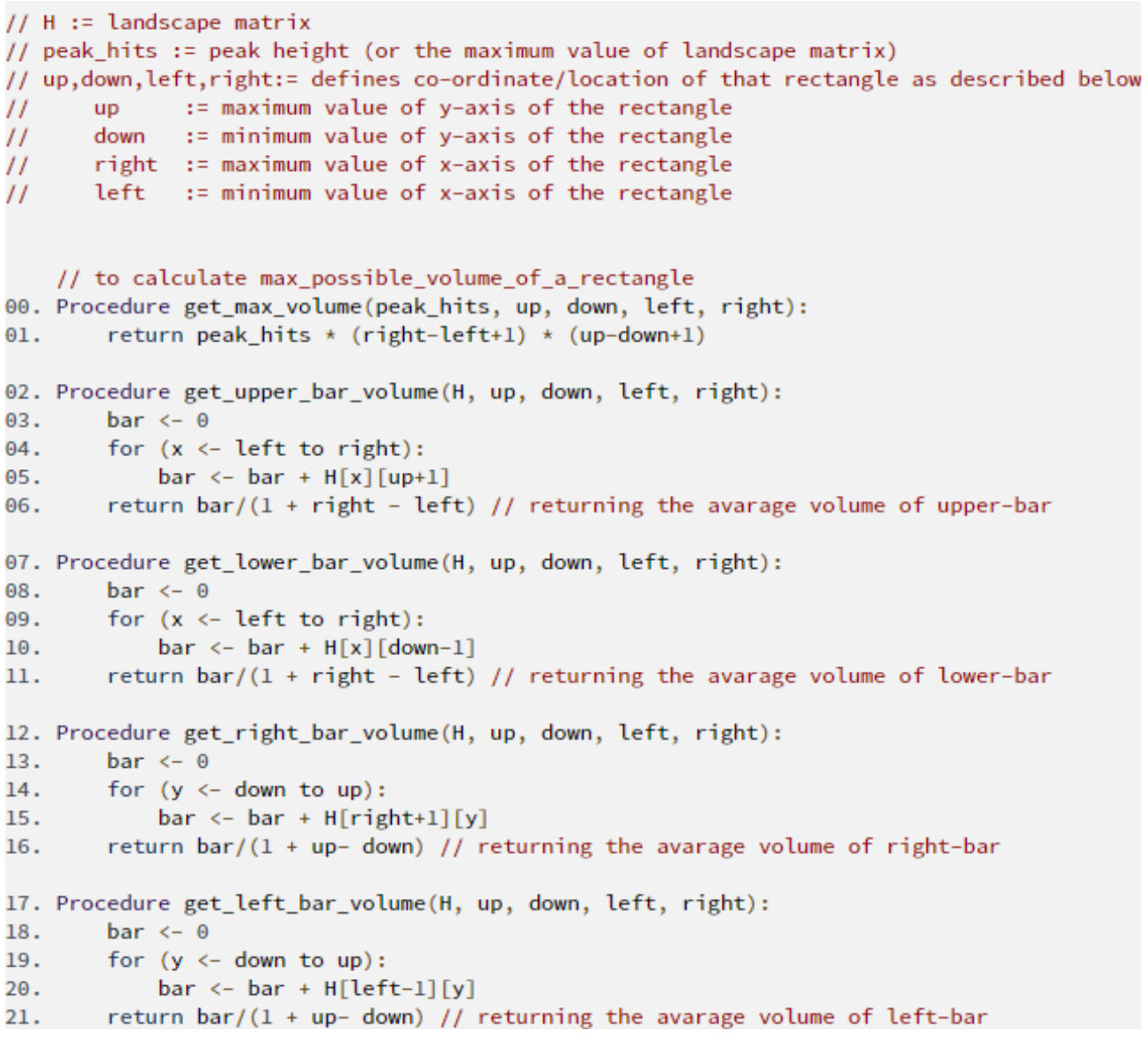

\section{Figure 19 : PANORAMA helper functions}


Now that we have defined the necessary components of algorithm, we can start talking about how a rectangle expands during algorithm's execution. Recall that PANORAMA is inspired to be very similar to WALK. Therefore, here also, we begin by identifying the location of the global maximum value across the landscape as the first step, which is a rectangle containing just the peak. The maximum possible volume for this rectangle is same as the actual volume of rectangle (since it has only one index - the peak). From here, we consider all four directions in which the rectangle can grow by calculating average volume of upper-bar, lower-bar, right-bar, and left-bar. Like the greedy behavior of WALK, we take the bar having the highest value of average volume. Note that this bar is not necessary to have the highest total volume but the highest average volume. Not taking the absolute highest volume prevents us from always picking the bar that is larger in size. This concludes the process of how rectangle is expanded at each step.

The final step of PANORAMA is to decide when to halt the expansion of rectangle. We continue the iteration of expanding the rectangle by following the process described in the above paragraph. At each iteration, after expanding the rectangle, we compare the total volume of the current rectangle with maximum possible volume for the rectangle. We stop when the volume of rectangle reaches below a certain threshold, where threshold is some percentage of maximum possible volume of the rectangle in that iteration. Here, the best value of this threshold is something we are going to find by performing a parameteroptimization in the next section. Once such rectangle is obtained, we remove that part of the SW-landscape matrix from the next run, by assigning zero values to all the points inside the rectangle. We repeat the process in the remaining part of the landscape matrix until we 
get maximum of 3 such rectangles or exhaust the complete landscape matrix, whichever is reached earlier. These are called the 3 sites detected by running PANAROMA.

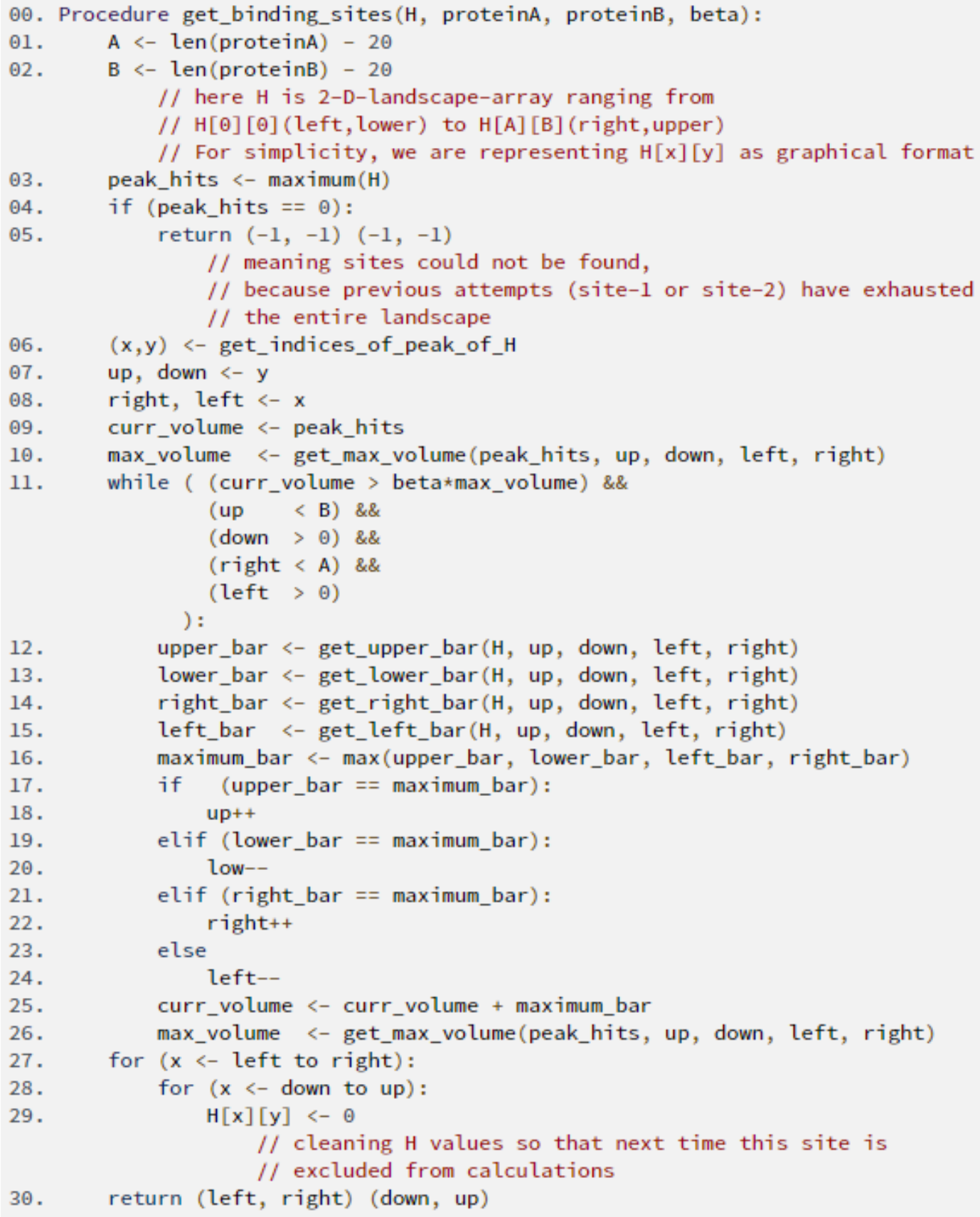

Figure 20 : PANORAMA main function 
The pseudo code to calculate PPI sites for PANORAMA algorithm is explained in Figure 20. We call the function explained from line 00 to line 30 , three times in the main applications where each time, it detects 1 site. Line 03 finds the global maximum value as the first step. Line 05 checks whether previous calls of the function have exhausted the landscape matrix and returns no sites (by returning -1) if they have. Lines 06 to 08 defines the first rectangle around the peak. Line 11 starts the iteration and expands this rectangle in each iteration, till the threshold is not breached and the rectangle is not expanded till boundaries of the landscape matrix. Lines 12,13 14 and 15 get the average volume for the four bars, upperbar, lower-bar, right-bar and left-bar respectively. Once the iterations are finished, either by finding out the sites or rectangle has expanded till the boundaries of landscape matrix, line 27 to 29 sets the values of landscape matrix 0 so that these values are not picked up in the next call of the function. Finally, it returns the site at line 30.

Working of PANORAMA with an example is illustrated in Figure 21, where we are showing picking of just one site. Here we set the same threshold as we had in the example of WALK, which is $91 \%$ pf the peak. Now, PANORAMA start with picking the global maximum value, which is 100.0 at index $(2,3)$. Volume of this first rectangle is currently equal to maximum possible volume. We have average volume of the upper-bar as 15.0 , average volume of lower-bar as 90.0, average volume for right-bar as 96.0 and average volume of left-bar as 80.0 Clearly, we have the right-bar with highest average volume hence we expend the rectangle on right. The new rectangle has two indexes, $(2,3)$ and $(3,3)$. The maximum possible volume of this rectangle is 200 whereas the actual volume becomes $196.0=100.0$ $(2,3)+96.0(3,3)$, which is greater than 0.91 times maximum possible volume $(196.0>$ 
$200.0 * 0.91)$. Hence, we continue to next iteration. In this iteration the average volume of the upper-bar is 95.3 , average of $(1,4)$ and $(3,4)$. Average volume of lower-bar is 91.5 , average of $(2,2)$ and $(3,2)$. Average volume for right-bar is $92.0(4,3)$ and left-bar has average volume as $80.0(1,3)$. We expand the rectangle on the upper-side this time and again the total volume of this rectangle is still greater than 0.91 times maximum possible volume.

The movements illustrated by arrows in Figure 21 shows that when we start from peak (2, 3), we make in total 9 moves before halting, in this order: (1) right, (2) up, (3) right, (4) up, (5) down, (6) right, (7) up, (8) right and (9) down. The total volume of this rectangle is 2714.8 which is below threshold $\left(2714.8<3000.0^{*} 0.91\right)$ and hence we halt here.

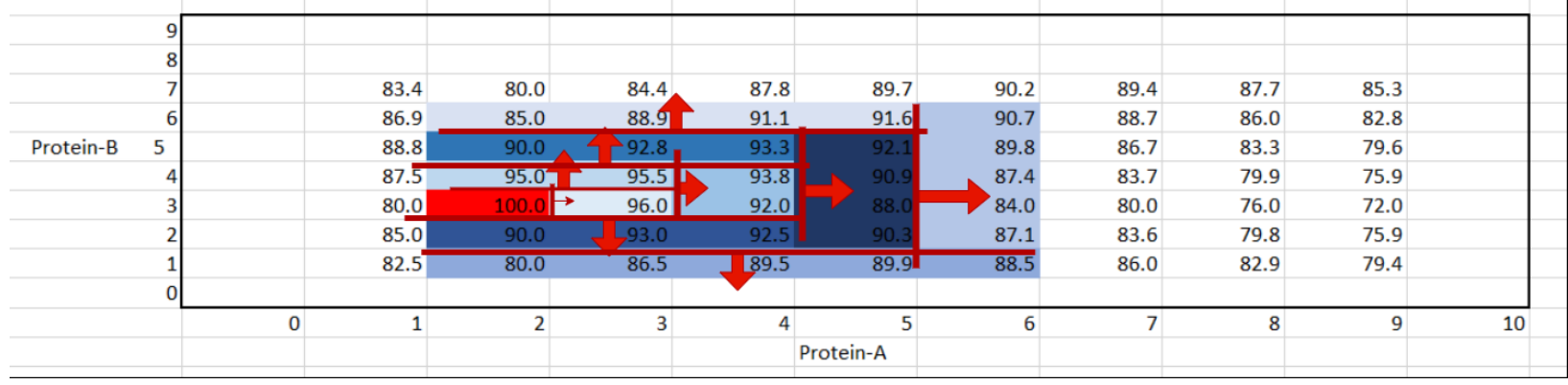

Figure 21 : PANORAMA technique - describing the panoramic view instead of linear walk

This final rectangle becomes the site detected by PANORAMA and is illustrated in Figure 22 by the surrounded blue box. 


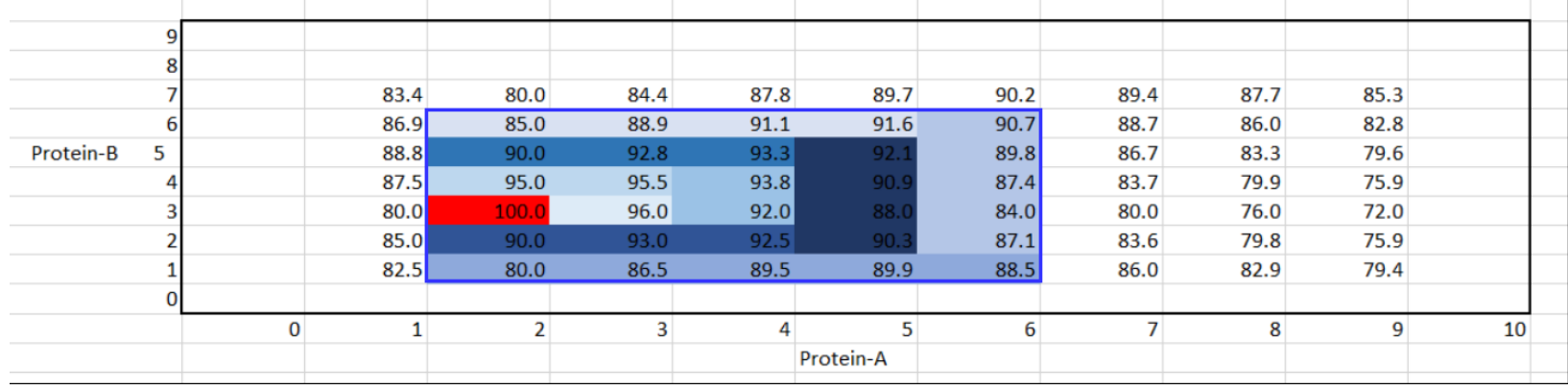

Figure 22 : PANORAMA site detection - does not miss diagonal entries

Since PANORAMA considers diagonal ridges, rather than focusing on just one AA like WALK does, this may lead to over-prediction of the PPI-site. However, since the ADM metric penalizes both under and over-predictions, using it to tune the parameters gives us the best parameter value that optimally regulates such false-positive predictions.

\subsubsection{Parameters Optimization}

As mentioned in the previous section, the best value for the threshold is obtained by undergoing the exercise of parameter-optimization. During parameter tuning for WALK, we performed such optimization by following Grid-Search Cross-Validation approach [66] [67] explained in detail earlier. Similar to WALK, here also, we perform 5-fold cross validation with the parameter-grid search of "threshold" value ranging from $10 \%$ to $90 \%$ (of the peak value), on a constant interval of $10 \%$, optimizing the lower values of ADM. Final box-plot distribution for ADM values for the best of the three prediction PPI-Sites, on all protein-pairs in illustrated with Figure 23. It demonstrates that higher values of the parameter seems slightly preferable over lower values of parameter, with the optimal parameter value coming out to be $50 \%$ of the peak. We also observed that at higher values of threshold, variability in the ADM values dropped. This could be because at higher 
threshold values, PANORAMA tries to expand into bigger rectangles before reaching threshold. And, while expanding to bigger rectangles, PANORAMA tends to make squarelike shapes. Hence, with increasing threshold values, it makes more square-like shapes while trying to predict the entire landscape image as a site.

PANORAMA algorithm parameter tuning on SW-landscapes

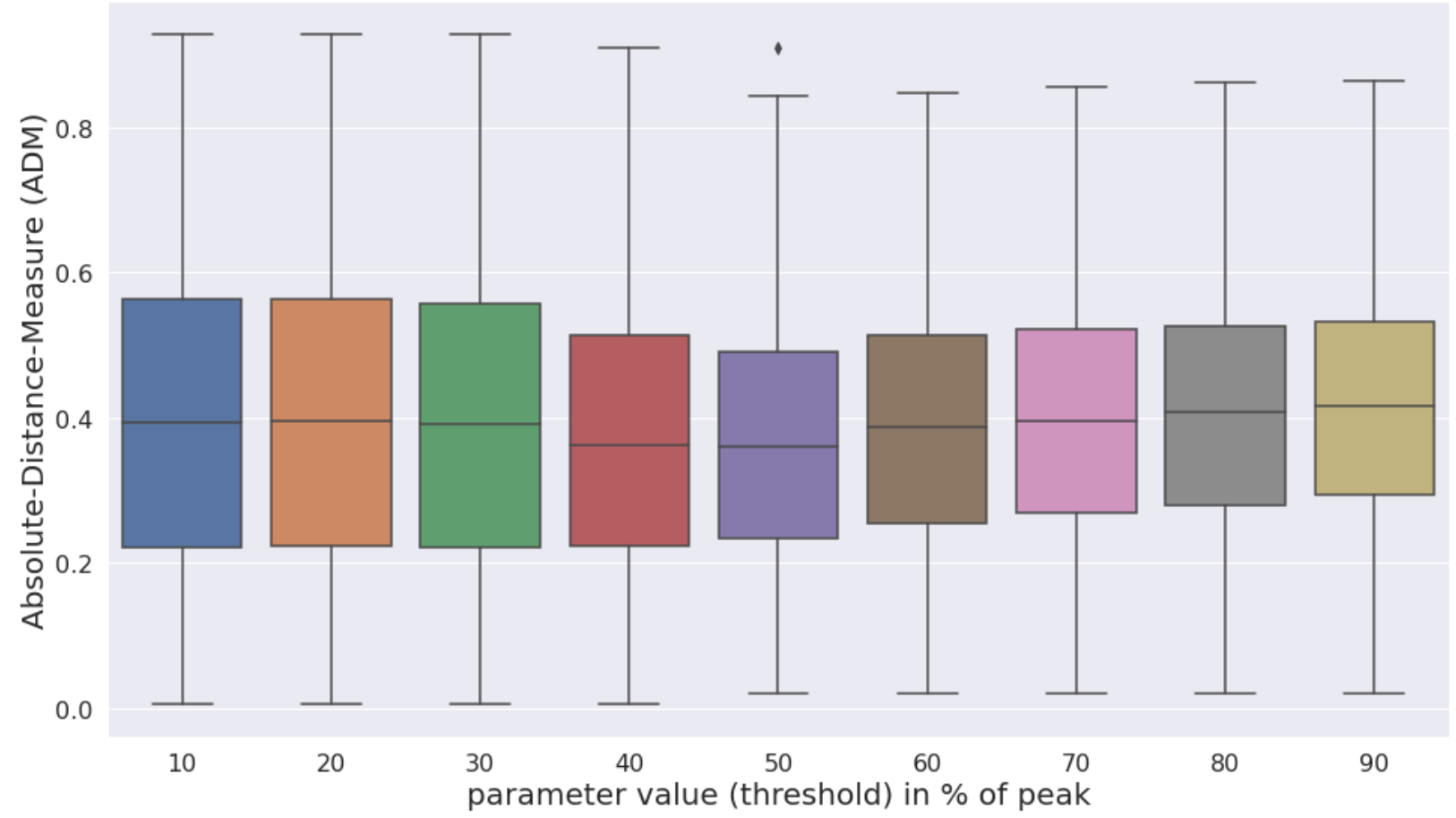

Figure 23 : PANORAMA parameter tuning

\subsubsection{WALK vs. PANORAMA}

While comparing the ADM distribution of PANORAMA with its best parameter, $50 \%$ of peak, to the WALK algorithm with its best parameter, $30 \%$ of the peak, we see an improvement in prediction of PPI-sites as shown in the Figure 24. The box plot distribution visualization, for the optimal parameter values of the algorithms, shows a decrease in ADM values for PANAROMA, clearly demonstrating its superior performance over WALK. 
Further tests for calculating statistical significance of differences will be discussed later in this chapter when discussing results of all the algorithms with each other.

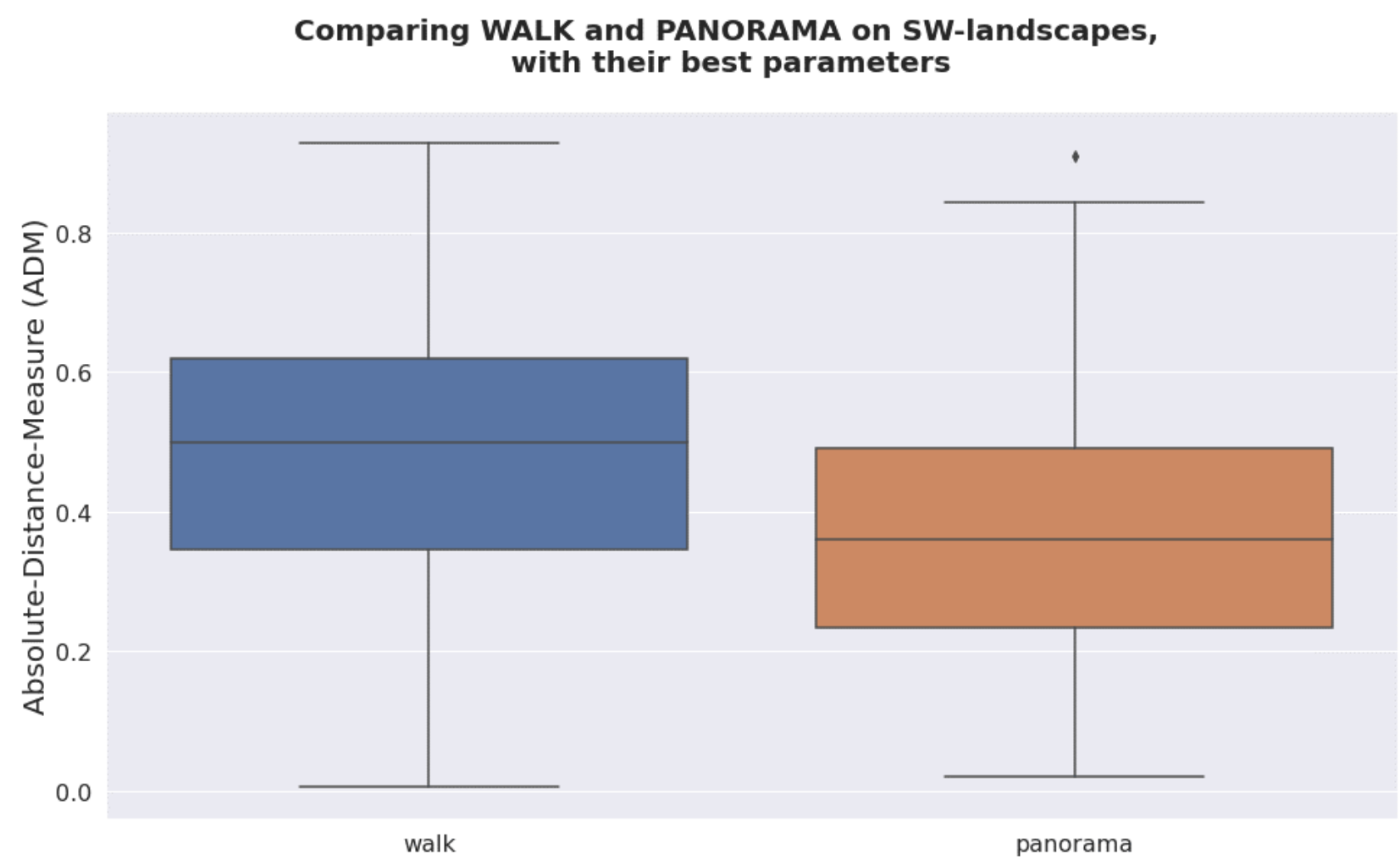

Figure 24 : Comparing PANORAMA and WALK on SW-landscapes, with their best parameter

\subsection{BRIGHT-SPOT Detection - Classical Image Segmentation}

Both the techniques discussed so far, the original WALK and the new PANORAMA, essentially consider the input SW-landscape as a matrix of scores, start with a global maximum (i.e., landscape peak), and then grow the size of predicted the PPI-sites using a greedy approach. Contrary to this, the BRIGHT-SPOT detection treats the landscapes as an image and implements various image processing techniques. Inspired by existing image 
processing techniques used for biological images [69], we made this shift of imagining the input SW-landscape as an image. Use of image-segmentation methods to identify the brightest segments in SW-landscape is explained in detail in the next section.

\subsubsection{Core Algorithm}

The BRIGHT-SPOT algorithm uses multi-stage processing of the input SW-landscape image, where the major steps are - blurring, applying a threshold, finding connected components, and lastly, calculating the minimum enclosing rectangle. The final rectangles are treated as the predicted PPI-sites and we perform a ranking to obtain a maximum of 3 PPI-Sites, workings of which is detailed in the upcoming sections. The workings in each of these seps is described as follows:

\subsubsection{Step-1: Adding Blur - A Gaussian Convolution}

Since the input SW-landscape is a two-dimensional matrix of scores, we can treat it as a two-dimensional image with one channel. For processing this input, if we normalize it to assign each pixel a value between 0 to 1 , we get a greyscale image. Furthermore, as a standard practice to reduce random noise in the greyscale image, we apply a low-pass filter. This is done by convolving the image with a Gaussian kernel, resulting in a blurred/smoothed image [70]. One thing to note here is that the dimensions of the image are not constant across all the input protein pairs. To take an example, for the input pair of ProteinA and ProteinB, of length $M$ and $N$ respectively, the dimension of SW-landscape would be $M x N$. This variability in dimension of the input image inspired us to various sizes of kernel for blurring. This becomes our first parameter that we tune to obtain the 
optimized performance of BRIGHT-SPOT detection. The details of these experiments are discussed in Section-4.3.2 - Parameter Optimization.

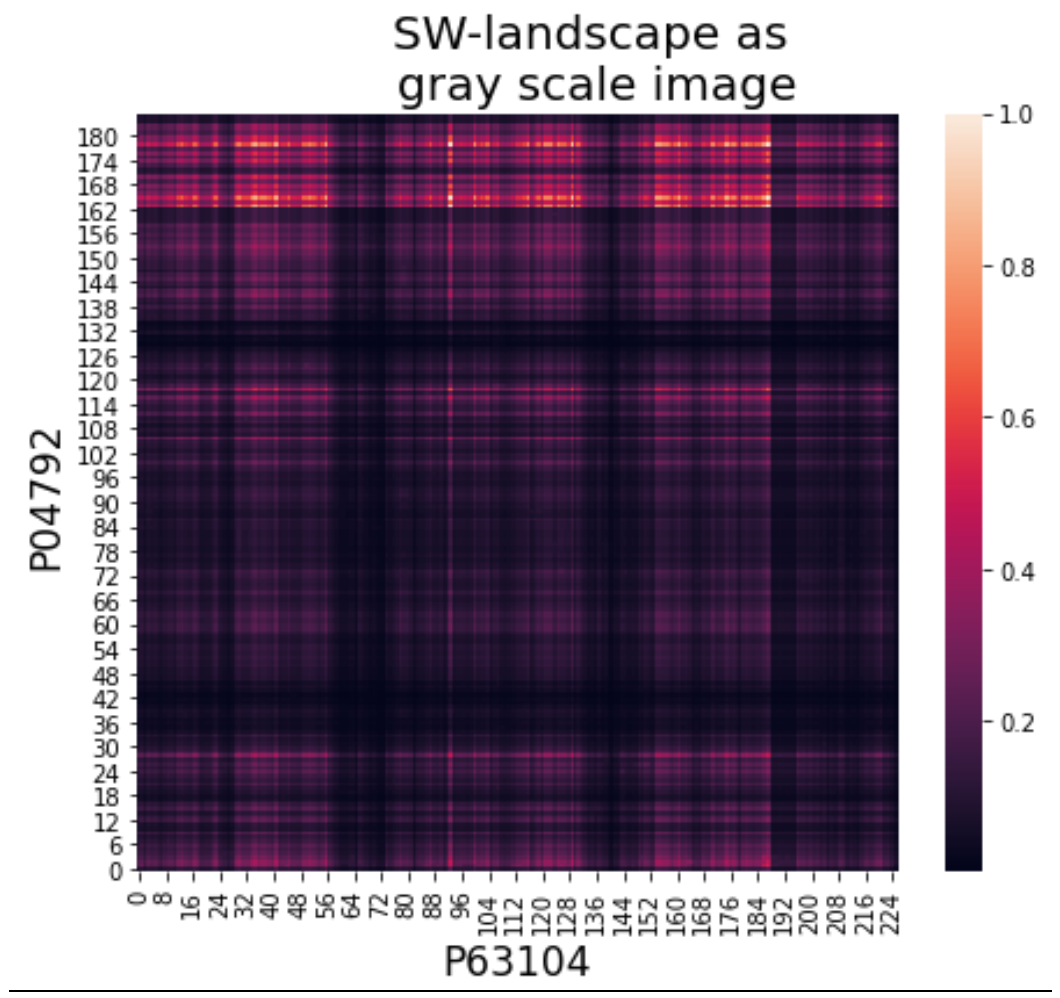

Figure 25 : SW-landscape matrix as gray scale image

Figure 25 illustrates a grayscale image for the input for protein pair - P63104 (on x-axis), P04792 (on y-axis), with protein length 245 and 205 respectively. Here we need to make a minor observation that landscape matrix is a direct output of PIPE, the length of axis is smaller than the length of the protein. For example, length of $x$-axis is $226=245-20+1$ where 20 is the window size in PIPE algorithm. Similarly, length of y-axis is 186. Now, applying just one Gaussian blur (of size $7 \times 7 \times 1$, chosen as per Section Parameters Optimization) resulting in Figure 26. 


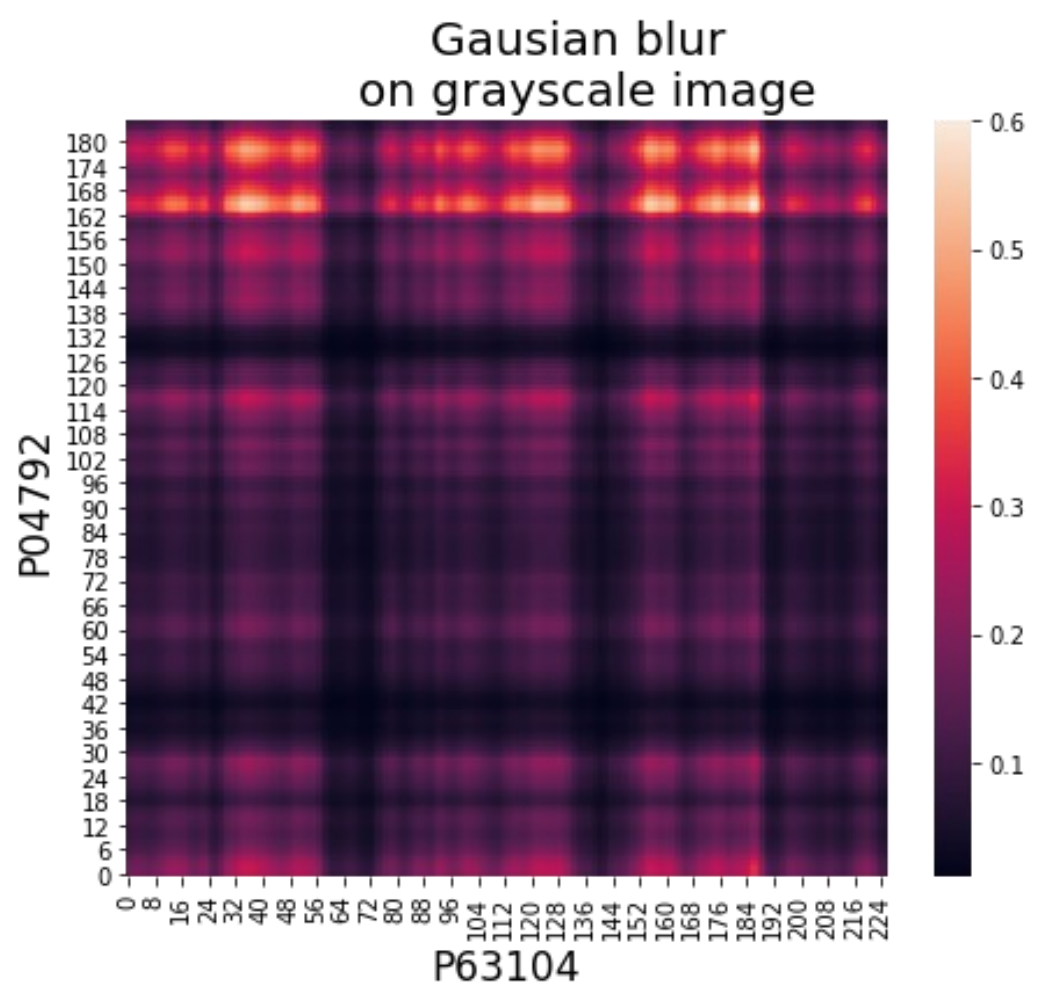

Figure 26 : Applying Gaussian Blur

\subsubsection{Step-2: Applying a threshold}

We apply a threshold to the output of the above step. Meaning that, all the pixels with value above this threshold, are set as 1 and rest are set as 0 . Here a pixel of value 1 means it is considered as a part of a bright spot in the next steps. Typically, threshold value is some percentage of the peak, and similar to the Gaussian kernel size, this is also considered as a hyperparameter of BRIGHT-SPOT detection. We performed the parameter tuning to get its best value and its details are described in Section Parameters Optimization. Continuing the same example, Figure 27 illustrates how applying the threshold looks like after blurring with the Gaussian kernel. Here a threshold value of " $30 \%$ of the peak" is applied (chosen as per Section Parameters Optimization). 


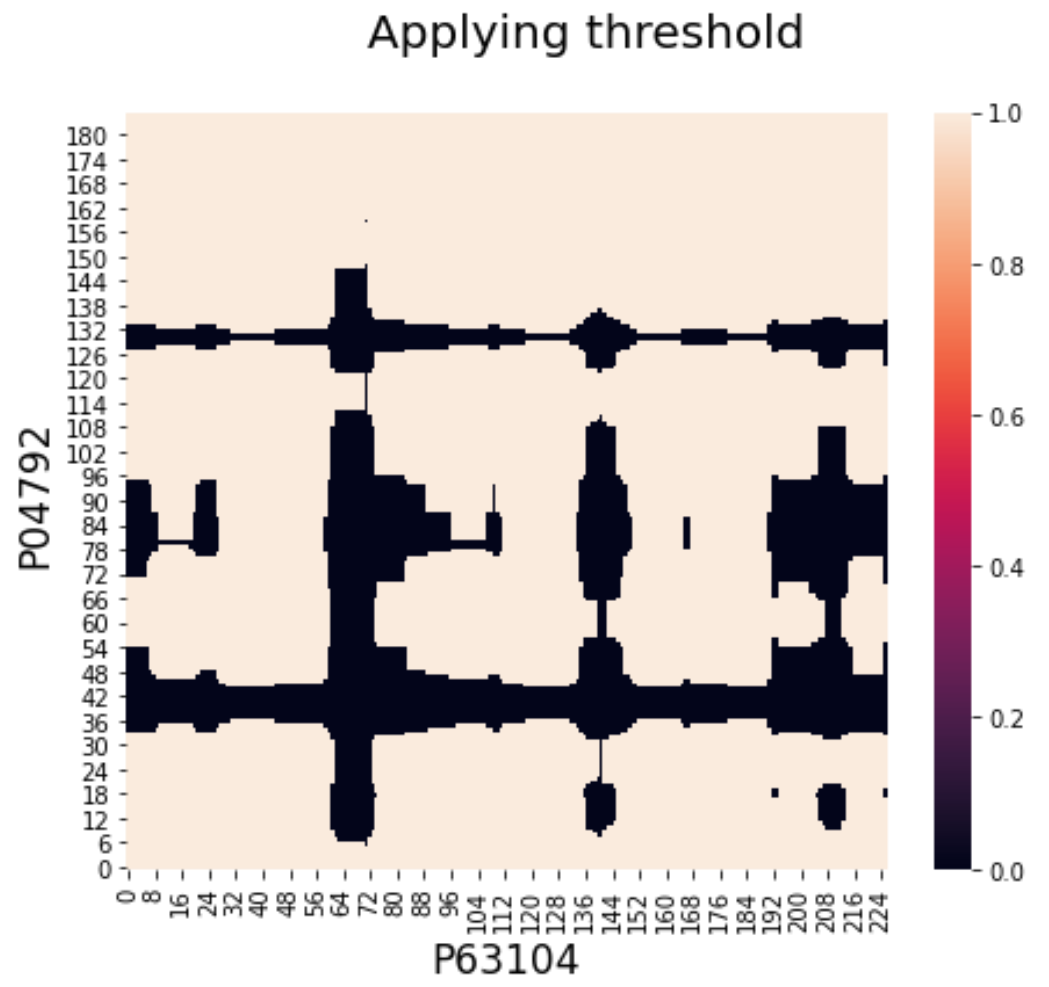

Figure 27 : Applying threshold after Gaussian Kernel

Figure 28 contains the main function that is called to get the final 3 PPI-Sites predicted by BRIGHT-SPOT detection. Notice that the input (at line 00) is not only the SW-landscape matrix but also the blur_size and the threshold_value. Both are parameters for which we aim to obtain optimum values. Line 02,03 and 04 performs normalization, Gaussian blur and threshold respectively, with the help of CV2 library functions [71]. Lastly, we call helper functions detailed at Figure 29 for detecting connected components (referred to as islands) followed by minimum enclosing rectangles as the final PPI-Sites as the final step. Both of these are explained in the next sections. 


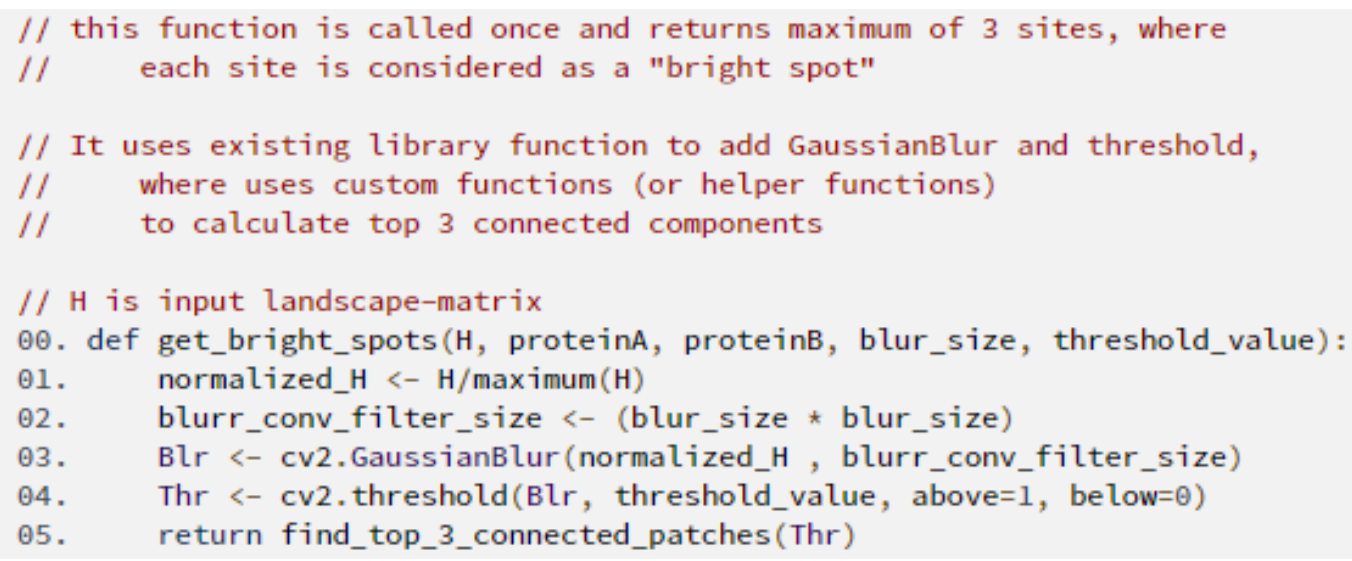

Figure 28 : BRIGHT-SPOT detection - Main function

\subsubsection{Step-3: Finding largest connected components - Segmentation}

After applying threshold, each pixel of the image is either 0 or 1 . We call pixel with 0 value a dark pixel and pixel with value 1 , a bright pixel. The aim in this step is to identify connected regions of bright pixels. A bright pixel is considered part of the surrounding component if at least one of the four neighboring pixels is also bright pixel. Here we only take the four directions namely up, down, left, and right to calculate the connected component. With this approach, starting with the first pixel, all the connected components are created. We consider these connected components as the brightest segments of the image (a.k.a. bright spots). We define the rank of these bright spot as well. To calculate the rank of a bright spot, we need to get the original pixel values from input SW-landscape. The bright spot containing the pixel representing the peak, highest value in SW-landscape, is considered the top ranked bright segment. Similarly, we rank the remaining bright spots.

We select top 3 segments by rank and process those for predicting PPI-Sites (explained in next section). The pseudo code in Figure 29 contains the helper functions. Here line 01 
calculates a graph by starting from a bright pixel and performs a graph traversal looking for directly connected bright pixels in up, down, right, and left directions. Line 02 contains the result of connected bright components referred as variable islands, where rank of each bright component (or island) has been described earlier in the section. Lastly, line 04 makes a loop over top 3 islands and calls for calculating the minimum enclosing rectangle for each island.

\subsubsection{Step-4: Calculating Minimum enclosing rectangle - Predicted PPI-Site}

For the 3 brightest segments (a.k.a. bright spots) by rank, we calculate minimum enclosing rectangle for each. These rectangles are finally called as the predicted PPI-Site by BRIGHTSPOT detection. For any bright segment, minimum enclosing rectangle is referred as an axis aligned rectangle that completely encloses the segment and is also the smallest in its size. As per the pseudocode described in Figure 29, function at line 06, we calculate such rectangle by getting the topmost, bottommost, rightmost, and leftmost point from the collection of 2-d points. These points define the boundary of the minimum enclosing rectangle which we refer to as predicted PPI interaction site. 
๑๑. def find_top_3_connected_patches(Img):

01. create graph from pixels of Img where each pixel can be connected to

02. a) maximum four neighbouring pixels (up, down, left, right) and

03. b) connection is only valid if the value of the neighbour is ' 1 '

04. islands <- isolated 'islands' of connected graphs got from above step

05. for each island <- reverse-sort(islands). top(3):

06. return bounding_rectangle(island) // defined below

๑7. def bounding_rectangle(points):

๑8. finds minimum enclosing rectangle for given set of 2 -d points

๑9. let

10. a) $(x, y)$ is coordiante of lower, left corner of this rectangle

11. b) $w$ is width of this rectangle

12. c)h is height of this rectangle

13. return $(x, x+w)(y, y+h) / /$ this is the site detected

Figure 29 : BRIGHT-SPOT detection - Helper functions

\section{Minimum enclosing rectangle 1}

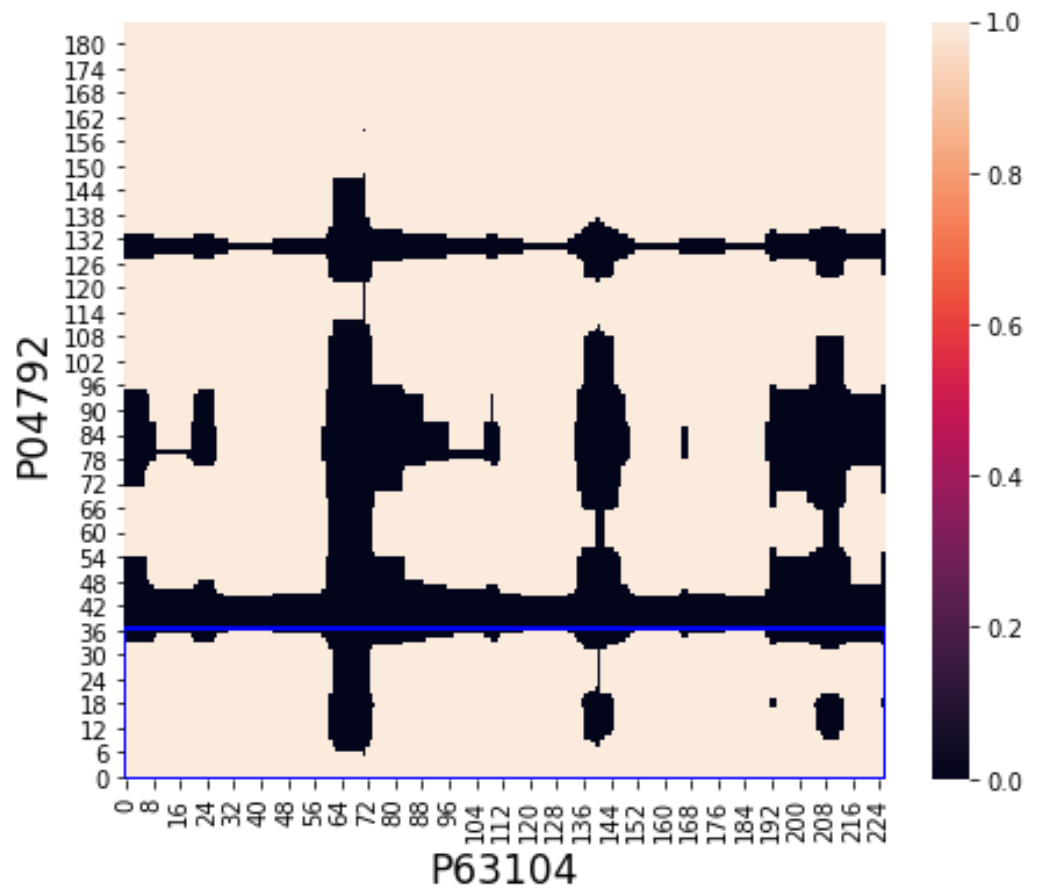


Minimum enclosing rectangle 2

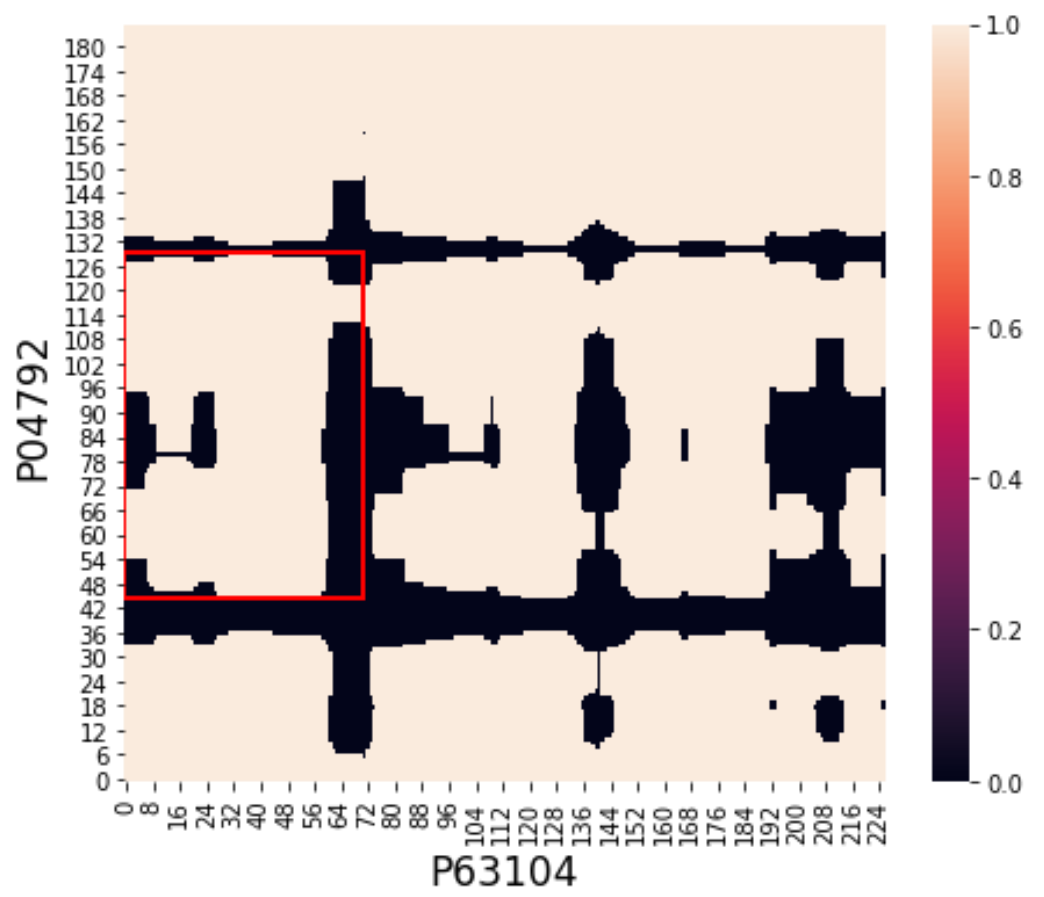

Minimum enclosing rectangle 3

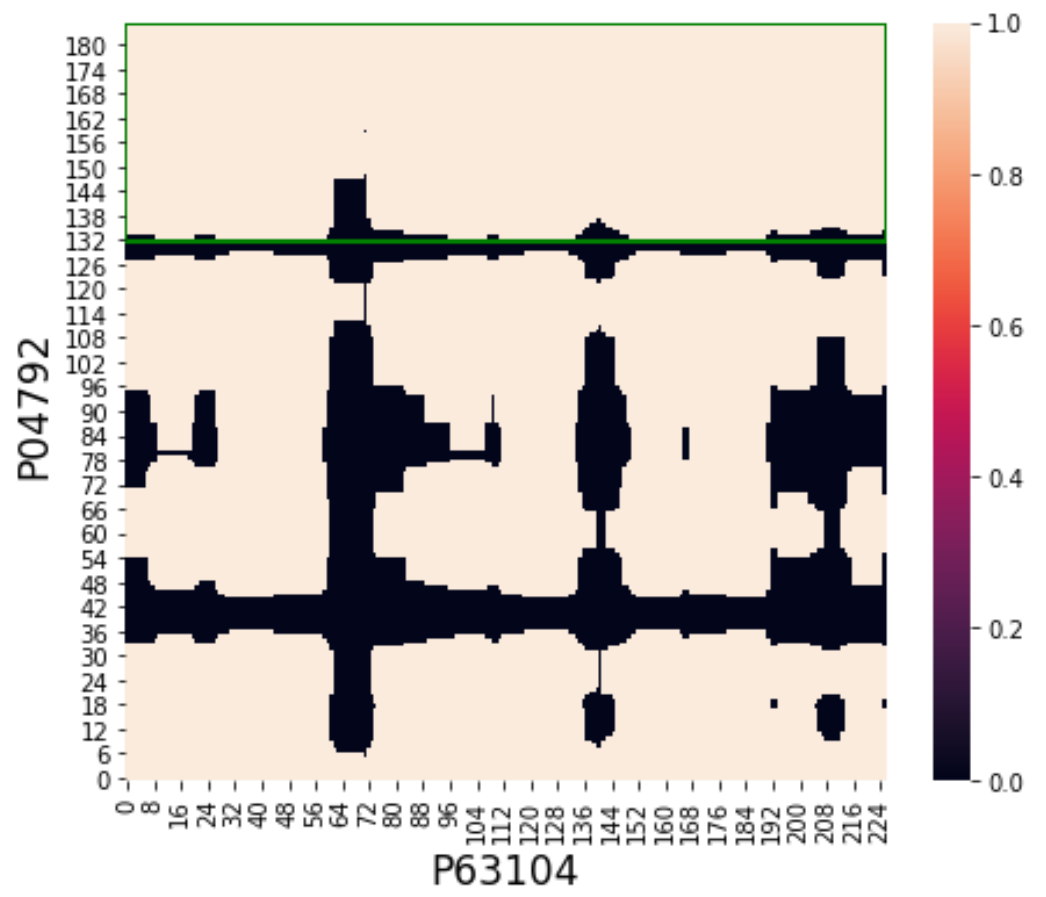

Figure 30 : Calculating Minimum Enclosing Rectangles on connected bright components (all three rectangles) 
Figure 30 illustrates finding of minimum enclosing rectangles for top 3 ranked connected bright components (on the example continued from Figure 27). These become the PPI-Sites predicted by BRIGHT-SPOT detection technique. A final comparison of these with the PPISite obtained in the lab verified validation data is illustrated at Figure 31

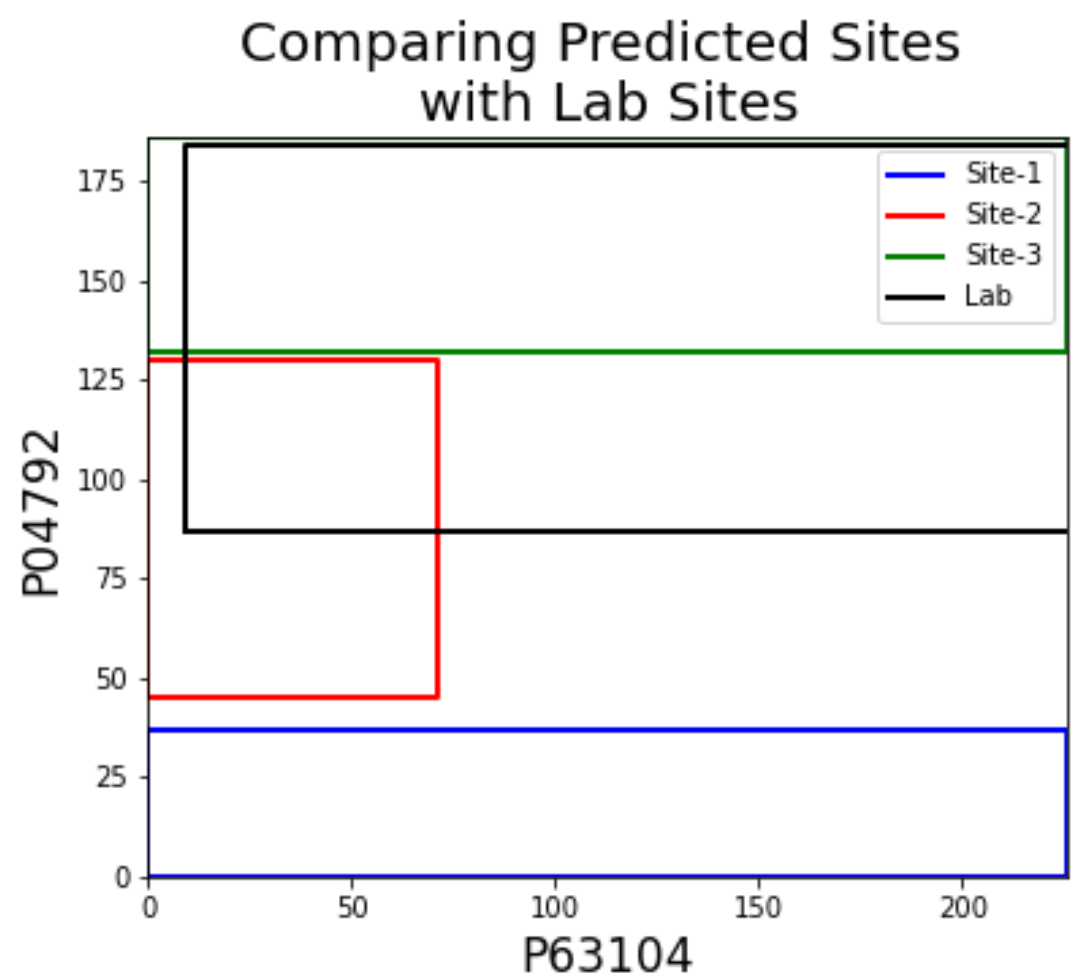

Figure 31 : Comparing BRIGHT-SPOT detection Sites with Lab verified PPI-Site

\subsubsection{Parameters Optimization}

BRIGHT-SPOT detection has two parameters - Gaussian kernel-size (described in Section 4.3.1.1) and threshold value (described in Section 4.3.1.2), which is generally some percentage of the peak value. Similar to WALK and PANORAMA, here also we perform grid search cross-validation [66] [67] for hyperparameter optimization. We executed a two-way grid search across kernel-size and threshold values and a final ADM distribution is 
illustrated as box-plot in Figure 32. Here x-axis represents different values of Gaussian kernel sizes we used in our experiments - one small kernel size (3x3), one medium kernel size (7x7) and one large kernel size (11x11). For each kernel size, we tried all possible values of threshold ranging from $10 \%$ of the peak to $90 \%$ of the peak, making it a two-way parameter-grid. The y-axis represents the ADM distribution of best of the three predicted PPI-Site, across parameter-grid of kernel sizes and threshold values. The lowest ADM values can be seen around threshold value 30\% across kernel sizes. A further narroweddown plot around this region reveals the best threshold value as $30 \%$ with kernel size $7 \mathrm{x} 7$, as illustrated in Figure 33.

\section{2-way Paramters sweep for BRIGHT-SPOT detection algorithm on SW-landscapes}

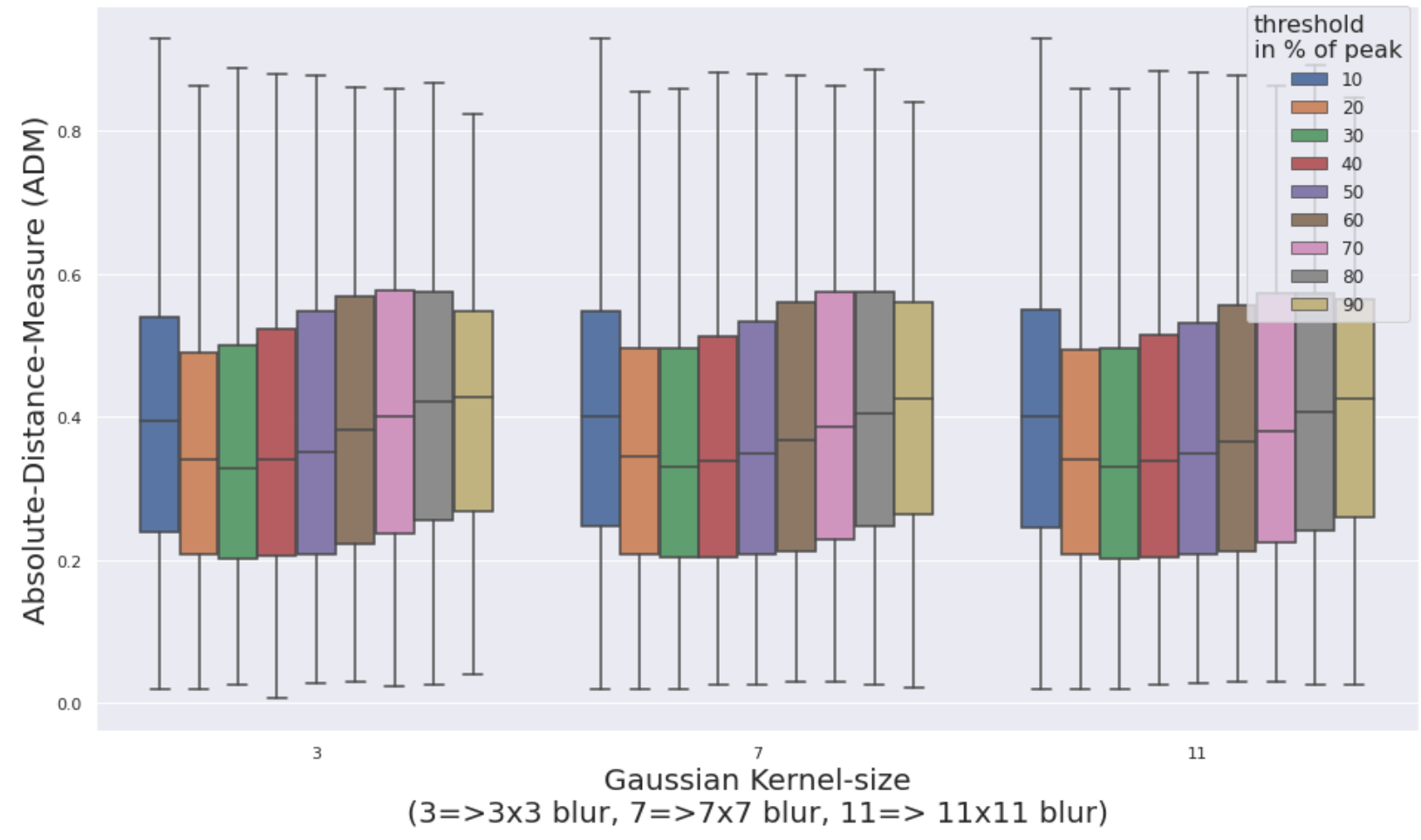

Figure 32 : BRIGHT-SPOT detection parameter optimization 
We concluded that the best parameters for BRIGHT-SPOT detection are - a gaussian kernel of size $7 \times 7$ and threshold value equal to $30 \%$ of the peak. The earlier Figure 31 illustrates the comparison of validation PPI-Site with the predicted sites obtained by applying these best parameters to BRIGHT-SPOT detection.

\section{Narrowing down Paramters-sweep}

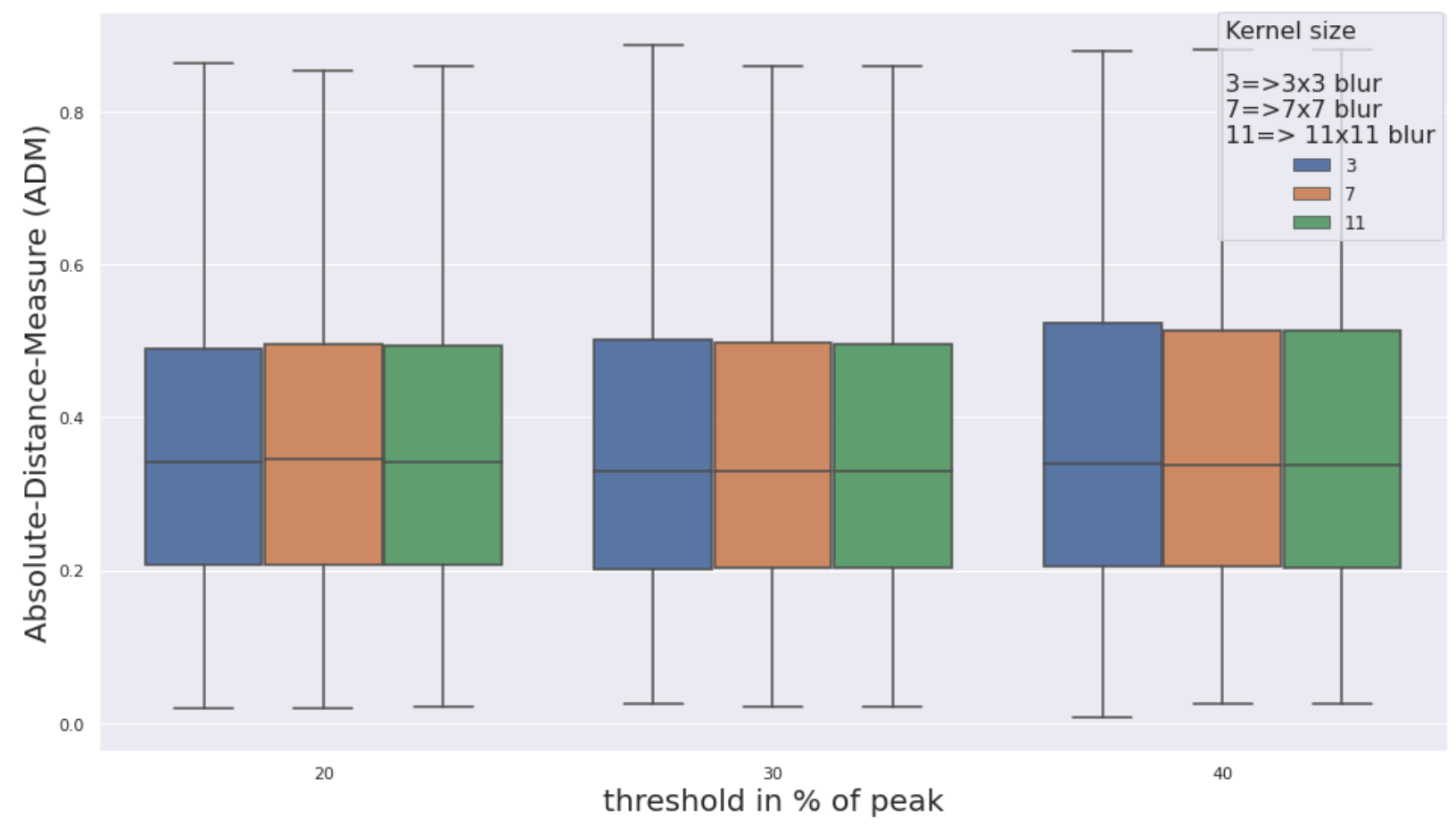

Figure 33 : Narrow Down Parameter Sweep

\subsubsection{WALK vs. BRIGHT-SPOT Detection}

While comparing the ADM distribution of BRIGHT-SPOT detection with its best parameters, 7x7 Gaussian kernel and threshold of $30 \%$ of peak, to the WALK algorithm with its best parameter, $30 \%$ of the peak, we see an improvement in prediction of PPI-sites as shown in Figure 34. The box plot distribution visualization shows lower ADM values for 
BRIGHT-SPOT detection demonstrating its better performance compared to WALK. Further statistical significance of differences will be discussed later in the thesis when discussing results of all the algorithms with each other.

\section{Comparing WALK and BRIGHT-SPOT detection on SW-landscapes, with their best parameters}

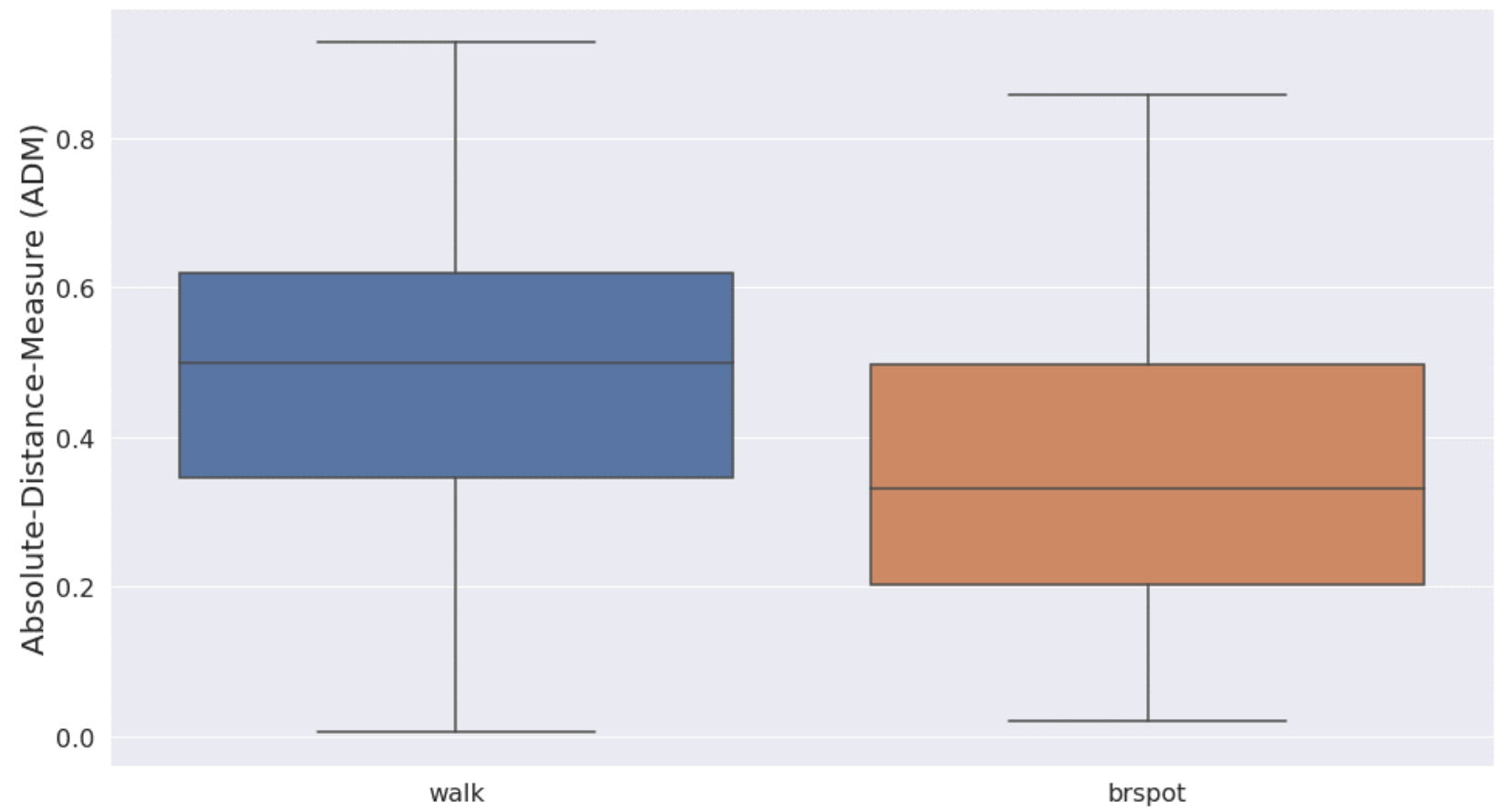

Figure 34 : Comparing WALK and BRIGHT-SPOT detection

\subsection{CLUSTER-NET - a Deep Learning approach}

Being inspired from the results of BRIGHT-SPOT detection, we continue exploring the idea of processing the SW-landscapes as images. Most of the state-of-the-art image processing techniques involve using some form of Convolutional Neural Networks (CNN) because these are very good at representing features for images. The kernels at each hidden layer in CNN helps creating better representation of input image which drastically improves the image processing tasks such as image classification [72], image segmentation [73] [74], and 
many more. Note that neural network training is a supervised learning approach that requires large volumes of training data. However, such labeled data would derive from lab validated PPI-sites, which are very short in supply for our experiments. Additionally, since the size of both the input SW-landscape image and the desired output PIPE-site are different for each training sample, additional image transformations would be required, introducing some information loss in order to generate uniform input and uniform labeledoutput. Thus, the traditional supervised learning with one deep neural network structure is not a viable option for our problem.

For these reasons and after observing the success of unsupervised methods during literature review [75] [76] [77], we introduce an unsupervised technique for training our CNN with customisable network architecture for each input. For example, if the input image size is 50x70 then in our neural network, number of neurons (or nodes) at the input layer would be set as 3,500, and at second layer it would be 350,000 (number of kernels are 100 at first layer). As stated earlier that we are not transforming the input image hence, some other input image with dimension $220 \times 310$ would require number of neurons in input layer to be set as 68,200 whereas at second layer number of neurons to be set would be $6,820,000$. This is the way we customize the network architecture for each SWlandscape image. Since the network architecture is different for each input, the parameters learnt for each input would be different from other input. For these reasons, we consider neural network, customizable for each input. 
Apart from network architecture being customisable, we have some fixed structures and parameters that are constant for each input image. For example, number of layers in each neural network, size of kernels at each layer, number of maximum segments, termination criteria, Gaussian kernel size post segmentation, and many more. We consider these as hyper parameters that control that behavior of CLUSTER-NET. We discuss the experimentations for hyper parameter tuning along with the challenges faced, in later section.

Now, we can briefly talk about the workings of CLUSTER-NET. For each input SWlandscape, a CNN is used to extract features for each pixel and then use these features to predict the class of the pixel, where each class signifies a segment. As explained earlier, we do not use the lab verified PPI-sites as labels to train the CNN, but use these to generate our own labels, using clustering on the original SW-landscape. A loss function is calculated by comparing the segments generated by the CNN with the clusters arising from the SWlandscape. The loss function is used to update the network parameters. Finally, lab verified PPI-sites are used to determine the absolute distance measure for each input protein pair. The following sections further describe this process in more details.

\subsubsection{Core Algorithm}

Similar to BRIGHT-SPOT detection, CLUSTER-NET also contains multiple stages to process the input SW-landscape image. The major steps are - generating features for each pixel using CNN, predicting the class of each pixel using these features, generating labels, using these labels to modify the parameters of neural network and finally, calculating the minimum enclosing rectangle. Just like in BRIGHT-SPOT detection, here also the final 
rectangles are treated as the predicted PPI-sites. The details for each of these steps is described as follows:

\subsubsection{Step-1: Generating Features - using Convolution kernels}

The aim for this step is to generate a feature representation for each pixel. Features generated by these steps are then used by a multi class classifiers to predict the class (or segment) for a pixel. We start with input SW-landscape of dimension $H \times W W$. This landscape can be treated as a greyscale image with dimensions $H \mathrm{x} W \mathrm{x} 1$. We then pass it through a convolutional layer with a kernel of size $K x K$. Here the size of kernel determines how many neighbouring pixels we want to consider in the feature representation of a pixel. This could be treated a one hyperparameter which is discussed in further section.

Note that applying each such kernel to the entire image would be responsible for generating one output channel each. Hence, having a total $F$ kernels would result into $F$ output channels. To keep the first two dimensions of input image same in output, we need to add half padding, resulting in output image of dimension $H \mathrm{x} W \mathrm{x} F$. This output image of dimension $H_{\mathrm{x}} W_{\mathrm{x}} F$ can be treated as image being represented into $F$ dimensions where each dimension signifies one feature space. Another way of looking at this output image of dimension $H \mathrm{x} W \mathrm{x} F$ can be as an image of dimension $H_{\mathrm{x}} W_{\mathrm{x}} 1$ with each pixel having a total number of $F$ features. This explains the usage of convolution kernels for pixel's feature representation.

Adding just a convolution layer with $F$ kernels of size $K x K$, performs a linear transformation on input $H \mathrm{x} W \mathrm{x} 1$ image which is not sufficient for classification of the pixels yet to be 
performed in next step. Hence, we introduce non-linearity by adding a layer of ReLU function. On top of bringing computational simplicity and handling vanishing gradients, ReLU is observed to provide better convergence performance over the sigmoid activation function for deep networks [78]. Finally, we apply batch normalization so that the output is normalized, with mean close to 0 and standard deviation close to 1 . This is now ready to be the input for the next layer. The mathematical reasons behind applying a batch normalization layer before the input to the next layer are discussed in detail in [79]. Note that adding both $R e L U$ and batch normalization does not change the dimension of the input image hence the dimension of the final output image would $H_{\mathrm{x}} W_{\mathrm{x}} F$.

\subsubsection{Step-2: Predicting class using these features - A Multiclass Classifier}

This step starts with an input $H_{\mathrm{x}} W_{\mathrm{x}} F$ image which, as explained earlier, can be interpreted as an image of size $H \times W_{x} 1$ with each pixel having $F$ features. If we apply a multiclass classifier, with each pixel having $F$ features, it then classifies each pixel into $C$ classes. Meaning that the output of this classifier would be an image of dimension $H_{\mathrm{x}} W_{\mathrm{x}} C$. Like earlier analogy, each pixel of the image with dimension $H \mathrm{x} W \mathrm{x} C$, now has $C$ values. However, here each of the value represents likelihood of the pixel belonging to that class. For example, if the pixel value of $5^{\text {th }}$ Channel is highest for some pixel, then that pixel belongs to $5^{\text {th }}$ class. Henceforth, $H \times W_{x} C$ means that we a maximum of $\mathrm{C}$ classes.

Now, to obtain an output of $H \mathrm{x} W \mathrm{x} C$ from an input image of $H \mathrm{x} W \mathrm{x} F$, we add a second convolution layer of $C$ kernels of dimensions $1 \times 1$ each, followed by a batch normalization layer. Finally, to determine the class of each pixel, we add a simple $\operatorname{argMax}$ to each pixel of this $H \mathrm{x} W \mathrm{x} C$ image. It gives us an output image of $H \mathrm{x} W \mathrm{x} 1$ that contains a natural number 
between 1 to $C$ for each pixel, representing which class each pixel belongs to. This concludes the classification of individual pixel of the input image. Note that we are doing $\operatorname{argMax}$ to the output of this layer, and given that $\operatorname{ReLU}$ is a monotonically increasing function, adding $R e L U$ will have no affect in results of $\operatorname{argMax}$. Hence, we are not adding $R e L U$ in this layer for computational time efficiency.
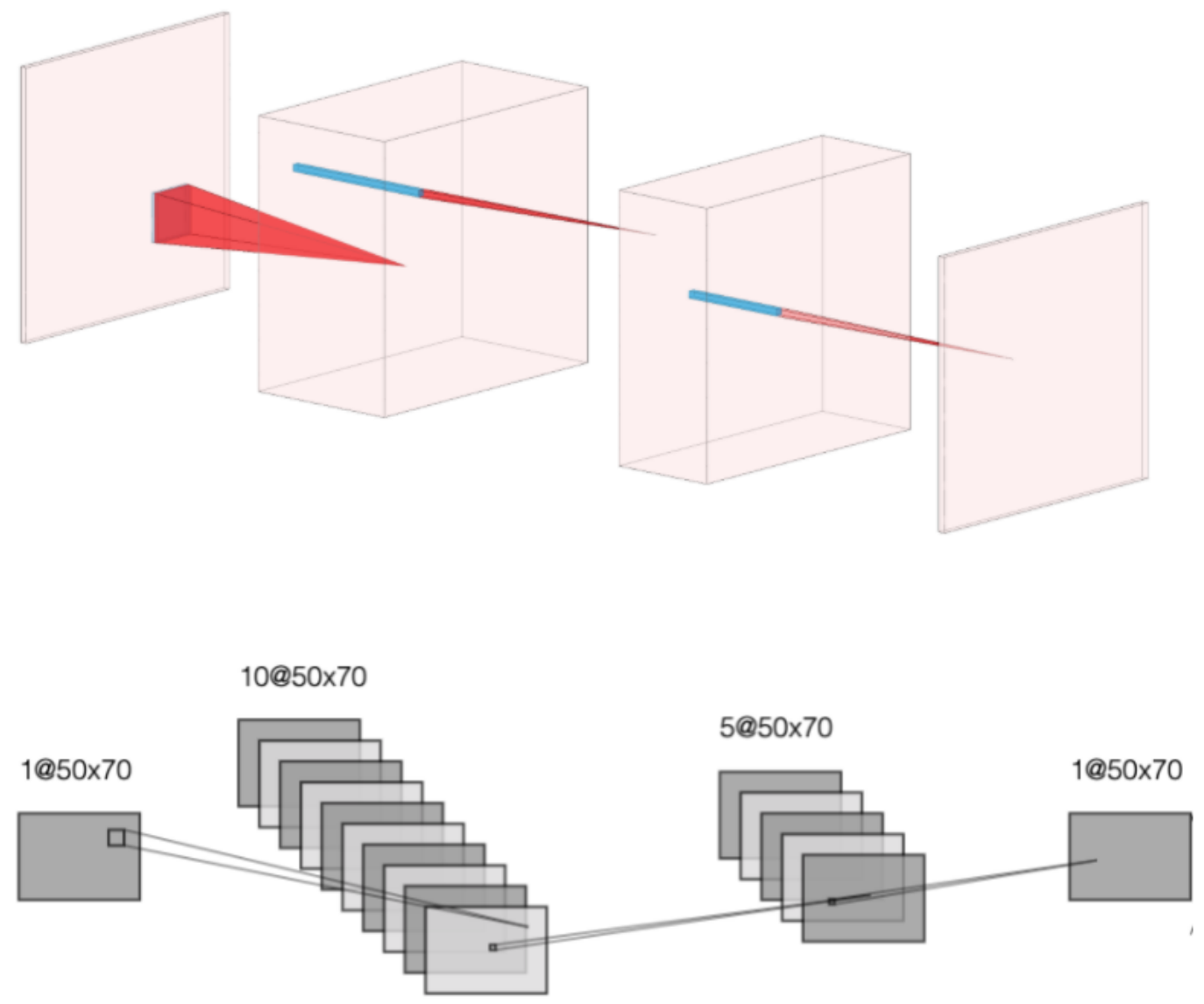

Convolution

Convolution

$\operatorname{Arg}-\operatorname{Max}$

Figure 35 : CLUSTER-NET Architecture 
To summarize these two steps, we take an input image of $H \mathrm{x} W \mathrm{x} 1$ and pass it to a convolution layer of $F$ kernels, each of size $K x K$, followed by a $R e L U$ layer, and a batch normalization layer. This results an output image of dimension $H \times W_{\mathrm{x}} F$ which is passed to next convolution layer of $C$ kernels each of size $1 \times 1$, followed by a batch normalization layer resulting into an output image of dimension $H_{\mathrm{x}} W_{\mathrm{x}} C$. After applying a final $\operatorname{argMax}$, it produces an $H_{\mathrm{x}} W_{\mathrm{x}} 1$ image which can now be looked like an image with a maximum of $C$ segments. This architecture is illustrated in Figure 35. Here the input image is of dimension $50 \mathrm{x} 70 \mathrm{x} 1$ (represented as $1 @ 50 \mathrm{x} 70$ ). The first layer has $F=10$ kernels of size $K \mathrm{x} K=7 \mathrm{x} 7$, resulting into a 50x70x10 image (represented as 10@50x70). The second layer uses these 10 features to perform multiclass classification for maximum of $C=5$ classes. Hence, it has $C=10$ kernels of size $1 \times 1$ resulting into output image of dimension $50 \times 70 \times 5$ (represented as 5@50x70). The final $\arg M a x$ layers puts the index of the class with the maximum value for each pixel resulting into output image of dimension 50x70x1 (represented as 1@50x70). 
1 channel input image $(186 \times 226 \times 1)$

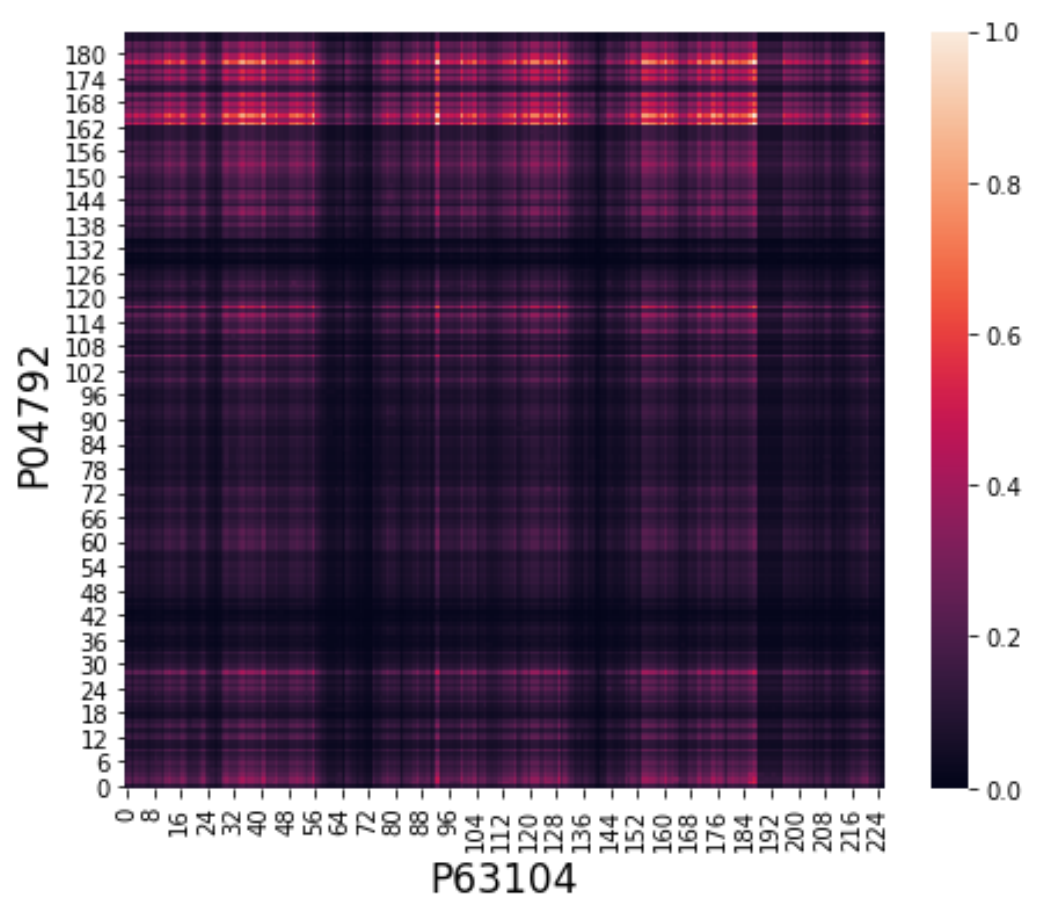

Figure 36 : SW-landscape matrix as 1 channel input image (of dimension $H \times W_{\times} 1$ )

Figure 36 illustrates an example of SW-landscape of protein pair - P63104 (on x-axis), P04792 (on y-axis), that was used to describe BRIGHT-SPOT detection algorithm. Since it is a 1 channel image, we can represent it as a heat-map image. We pass this image to the convolutional neural network, with architecture described above, and obtain the final output after argMax. Figure 37 illustrates the final output image. For the first layer, we picked the kernel size of $7 \mathrm{x} 7$ with a total of 100 kernels. The second layer has 50 kernels of $1 \times 1$ (creating a multiclass classifier) outputting to final image with each pixel belonging to one of the 50 maximum predicted classes. These parameter values are discussed in later section. We can faintly see the input image's pattern in Figure 37 and clearly this network needs feedback to perform a better segmentation task. 


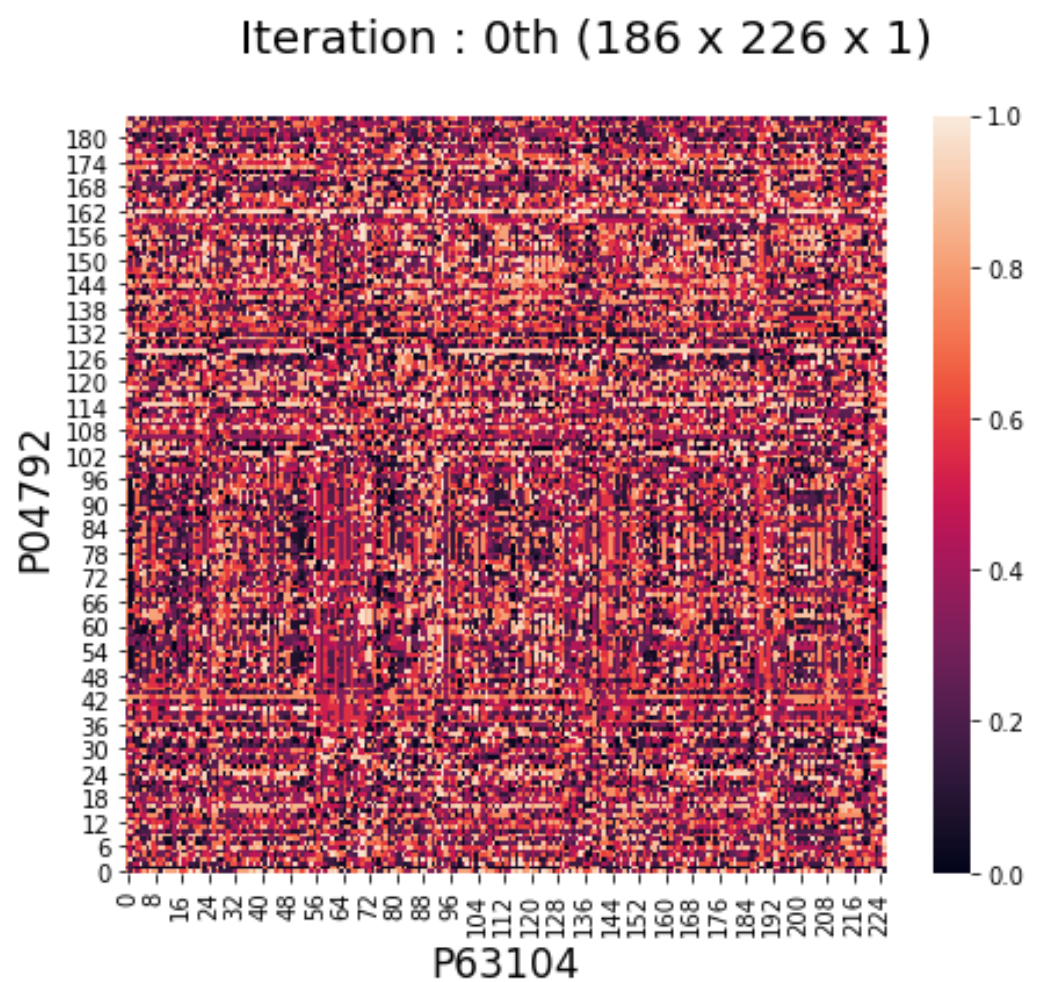

Figure 37 : Passing input image to Convolution Neural Network

\subsubsection{Step-3: Generating Labels - using Simple Linear Iterative Clustering}

Once we generate the predicted class of each pixel, we now need the actual labeled-class for each pixel to calculate the loss, which is then used to update the parameters of the above neural network. First, we will discuss state of the art unsupervised image segmentation technique used on input, followed by how it is used for generating our labeled class for each pixel.

We are using a state-of-the-art clustering technique, Simple Linear Iterative Clustering [80] (SLIC), to cluster the pixels of the input image. We chose this technique because SLIC clusters pixels by balancing the pixel spatial proximity and similarity in actual pixel value. In other words, pixels of similar values and are also neighbours, will have the highest likelihood of being in one cluster. In the output generated by SLIC, every cluster of pixels 
can be treated as one class (or segment), and in this way SLIC is used for unsupervised segmentation of an image. The most significant parameter available in SLIC balances the importance of spatial proximity with pixel value. Figure 38 illustrates segmentation performed with various parameters of SLIC on the input image illustrated in Figure 36. For the output with large number of segments, SLIC puts high preference on location of pixels whereas for the output with small number of segments SLIC put high preference on pixel values. We have added an output with medium number of segments representing a balance of importance between spatial proximity and pixel value. In order to decide which parameter works best for our application, we need to understand how we are using this segmented output to generate our labels. 

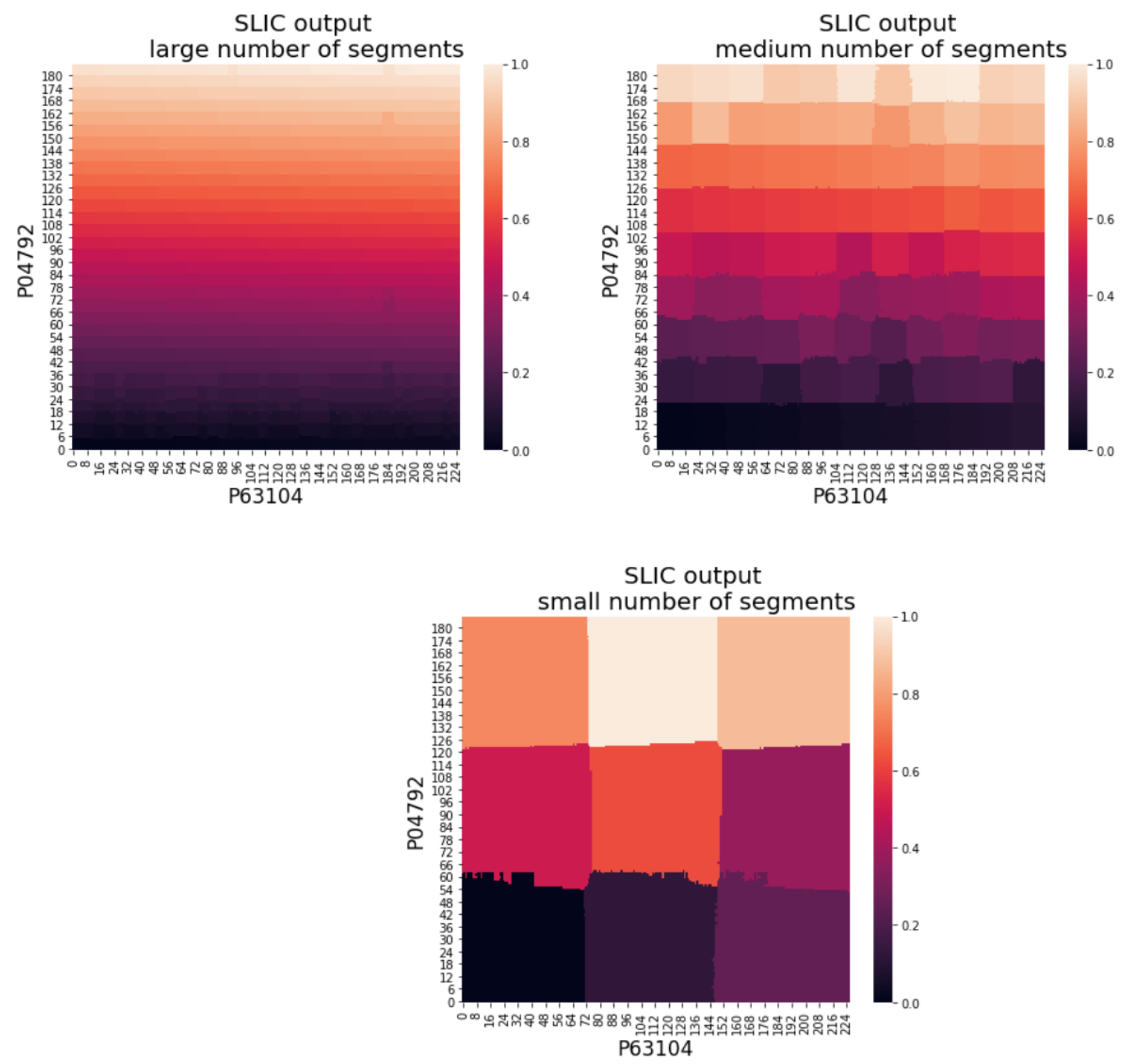

Figure 38: Applying SLIC to input SW-landscape image

Recall that in CLUSTER-NET output, the value at pixel is equal to the class id of that pixel. We can call this predicted class of the pixel. Similarly, each segment in SLIC output can be treated as one class. Now there are four possibilities for any adjacent pair of pixels. Use-case-1: pixel-pair belongs to same class in both CLUSTER-NET output and SLIC output. Use-case-2: pixel-pair belongs to different classes in both CLUSTER-NET output and SLIC output. Use-case-3: pixel-pair belongs to same class in CLUSTER-NET output but different 
class in SLIC output. Lastly, Use-case-4: pixel-pair belongs to different classes in CLUSTERNET output but same class in SLIC output. In the first two cases CLUSTER-NET and SLIC agree with each other's segmentation output whereas in the remaining two, both disagree. Hence, for first two use-cases, we can say that CLUSTER-NET has predicted correct classes for the pixel-pair because both CLUSTER-NET and SLIC agrees on segmentation. For use-case-3, when SLIC output suggests pixel-pair belongs to different classes but CLUSTER-NET output predicted the same class for it, we trust CLUSTER-NET prediction. Hence, the labeled classes for both pixels are kept the same as the predicted classes in all the first three use-cases. Only in use-case- 4 where the pixel-pair is predicted to be in different classes (or segments) in CLUSTER-NET output, we change the labeled class of one of the pixels. The pixel from a smaller segment gets its labeled class value equal to the class id of predicted class of the other pixel. Here size of segment means number of pixels in that segment (or class). The labeled class for the pixel from the bigger segment is kept same as its predicted class. If we define "error" for a pixel when its labeled class is not the same as the predicted class, only the use-case- 4 contributes to the error count. We can summarize these decisions for all four use-cases by stating that if a pixel-pair belongs to a one class (or segment) in either of the outputs, it is kept in one segment while assigning the labeled class. We consider CLUSTER-NET output and SLIC-output equally important. If we consider SLIC output to be always correct, the CLUSTER-NET would be learning nothing, but the SLIC algorithm. In which case, we are better off with using just SLIC-output instead of running any CLUSTER-NET. On the other hand, if we consider CLUSTER-NET output to be always correct we do not have errors to contribute to learning the network since labeled 
class would be exactly same as predicted class. Hence it is necessary to consider CLUSTERNET output and SLIC-output equally.

Considering the approach of generating labeled class explained above, it is clear that false positives should be as low as possible. In SLIC output, a false positive example would be a pixel-pair that should not be in one segment but has a same class value in SLIC output. We favor generating more segments over fewer segments in SLIC output. Hence, we opt for the parameter which gives us a large number of total segments in the SLIC output, which is illustrated as the first image in Figure 38.

\subsubsection{Step-4: Update parameters until it converges - Actual Network Learning}

We obtain the labeled class for each pixel of the input image as explained in the previous step. These are compared with CLUSTER-NET prediction in order to calculate the loss and update its parameters accordingly. Since it is a multiclass classification problem, we use categorical cross entropy loss to learn and update the CLUSTER-NET parameters. Once we update the parameters on the input SW-landscape image, we again pass the same input image on the newly updated CLUSTER-NET. Note that even though SLIC output is not changed for this image, since the CLUSTER-NET output might change with the updated network parameters, the final labels might also change by following the strategy explained in the previous section to obtain the label. This backward propagation is continued until CLUSTER-NET hits the terminal condition. Since the terminal condition is dependent on the computational resources available, hence it is discussed during parameter optimization section. 
1 channel input image $(186 \times 226 \times 1)$

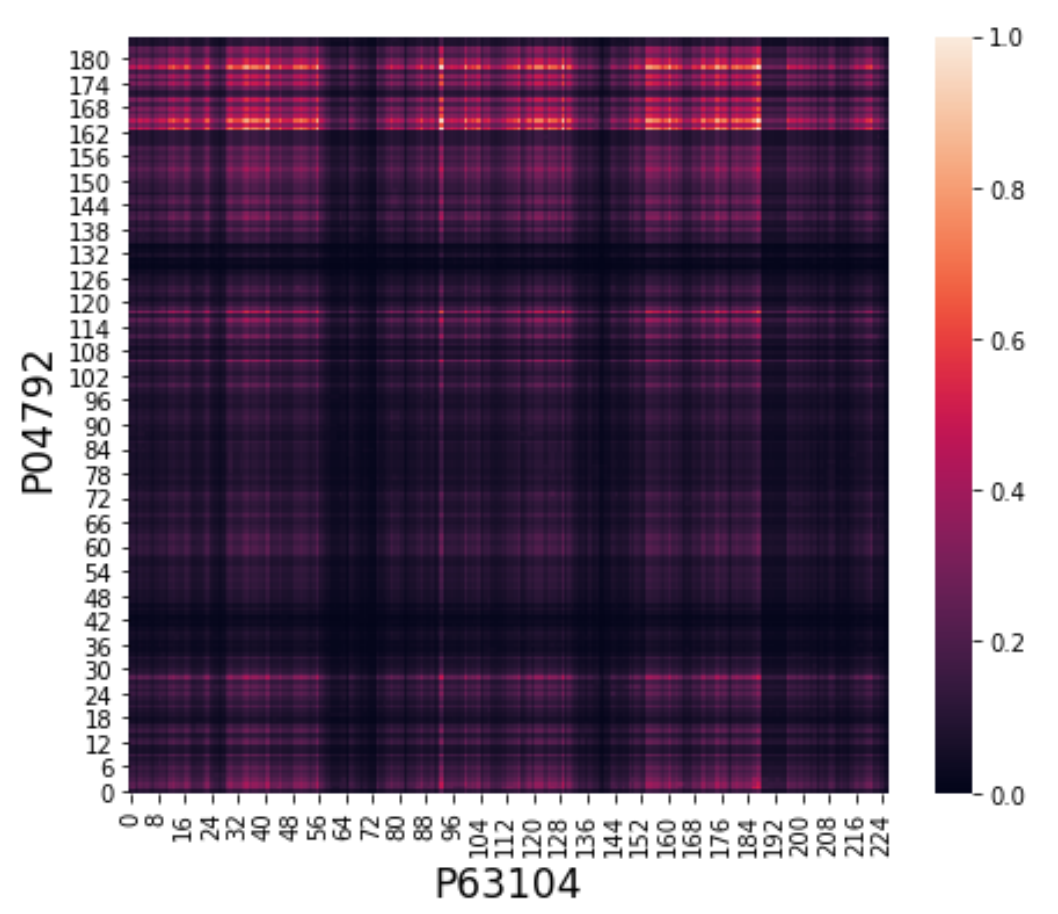

Figure 39: Input SW-landscape to CLUSTER-NET (recreation of Figure 36)

After sorting segments in descending order by their size, Figure 40 illustrates some of the top segments of final CLUSTER-NET output for the input SW-landscape shown in Figure 39. Segment-1 (with 23,578 pixels in the segment) looks like a segment representing the background, whereas segment-2, or segment-3 etc. are potential candidates for containing PPI-Site. Understandably, the segment representing the "background" or "floor" would be one of the largest segments. Hence, we sort the segments by their brightest pixel's value, in descending order. Meaning, the brightest segment, containing the peak, would be considered first for detecting PPI-Sites explained in next step.

Since the final output of CLUSTER-NET is an image where each pixel contains value equal to its final predicted class id, we need some transformation to generate images illustrated in 
Figure 40 . We are converting all the pixels of a class to 1 and rest all the pixels to 0 before plotting. For example, to generate image for segment-3, all the pixels with value "3" are converted to 1 and rest of the pixels are converted to 0 .
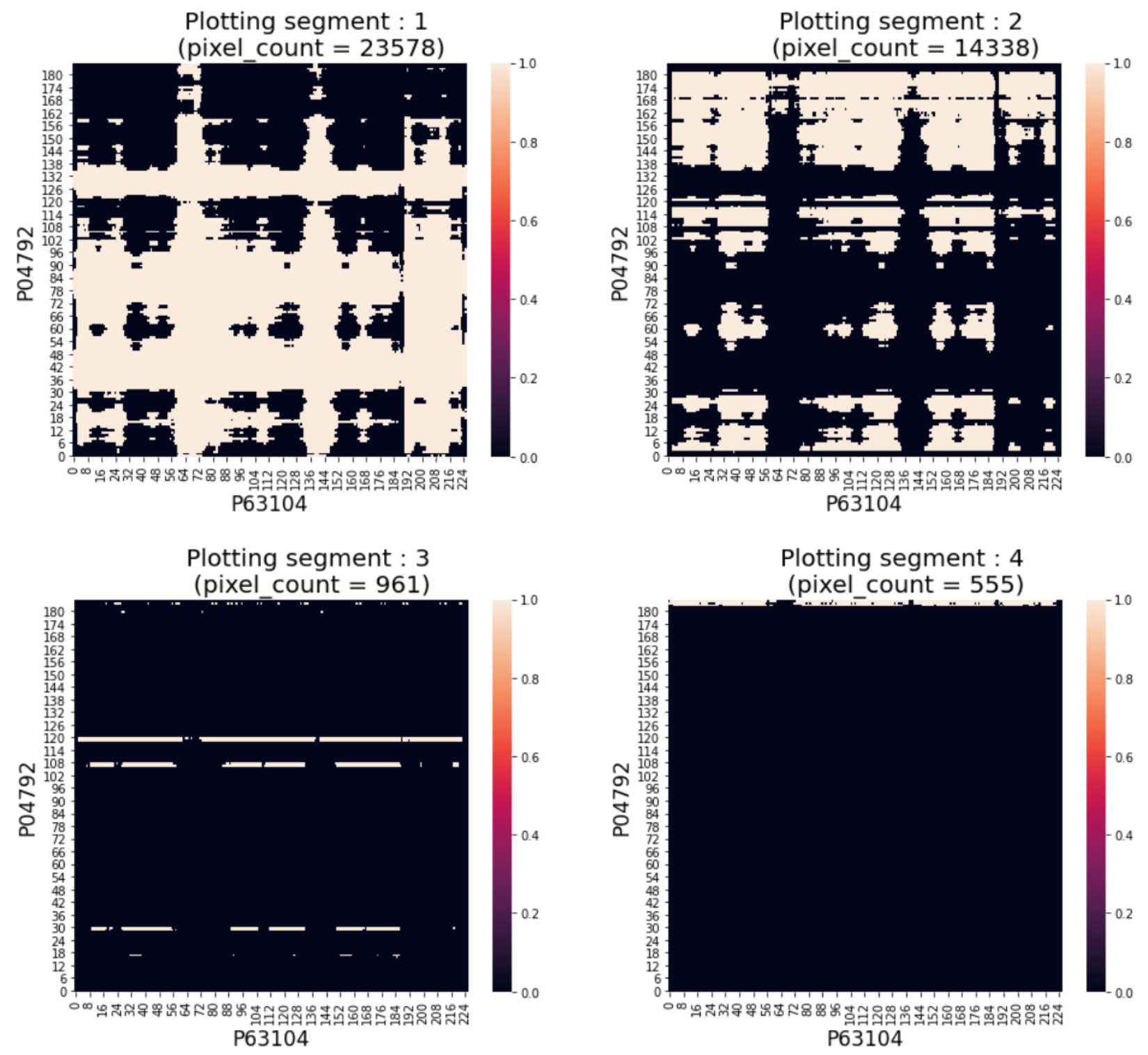

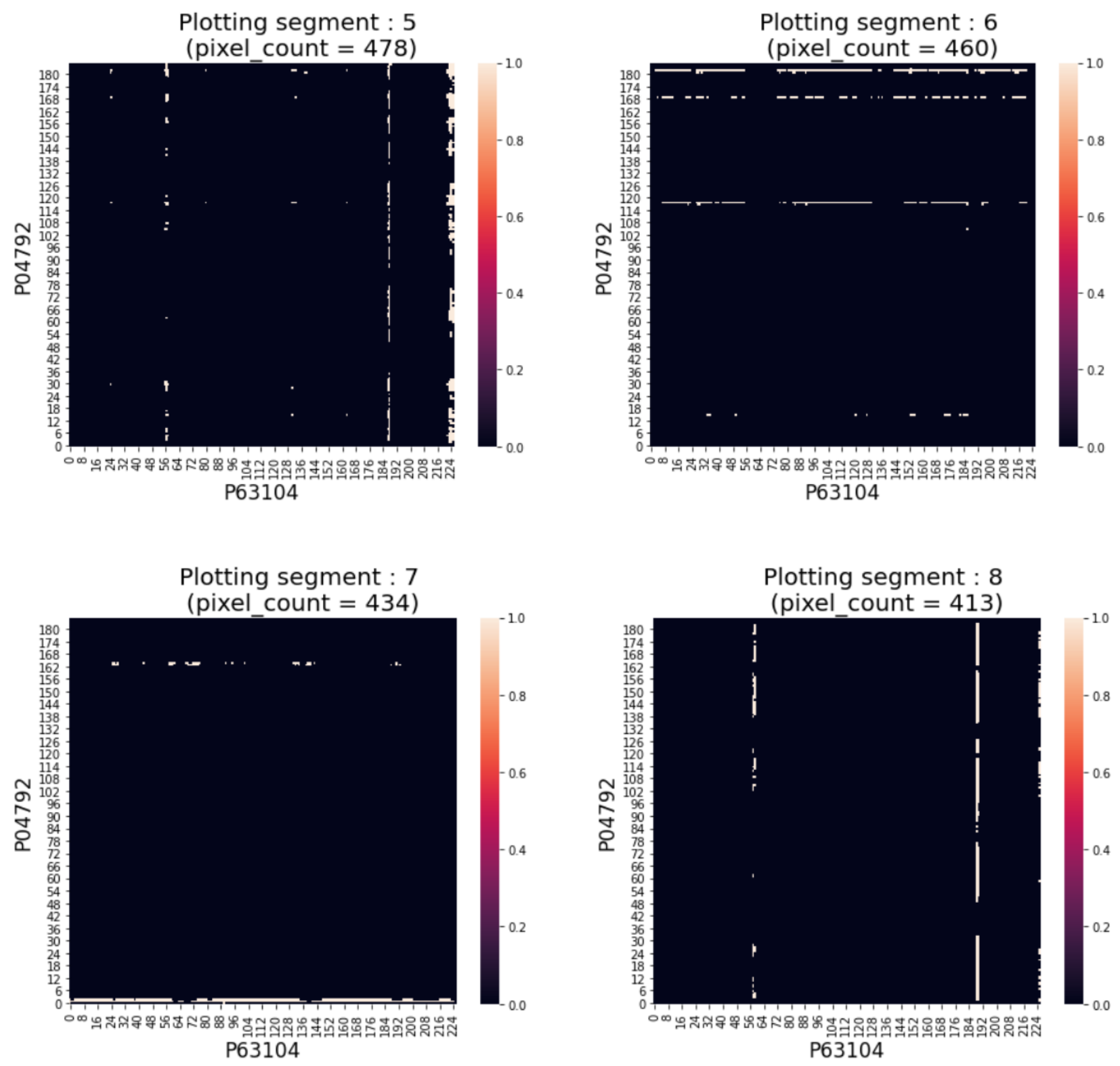

Figure 40: CLUSTER-NET top largest final segments (here plotting only top 8 segments for illustration)

\subsubsection{Step-5: Detect PPI-sites - Minimum Enclosing Rectangle}

Before proceeding, we add a tiny gaussian blur to smooth out the output of CLUSTER-NET to combat the overfitting tendencies of Neural Networks. Recall that the output of CLUSTER-NET is an image segmented into various classes. As mentioned in previous step, 
we sort these segments by their brightest pixel's value, in descending order. Note that a segment need not be contiguous until this point. Hence, we find the contiguous connected components within the segment and further rank them by their brightest pixels exactly like we do in BRIGHT-SPOT detection. Finally, for a continuous component, we calculate the minimum enclosing rectangle and call it as likely PPI-Site. We continue till we find at most 3 PPI-Sites or exhaust all the segments.

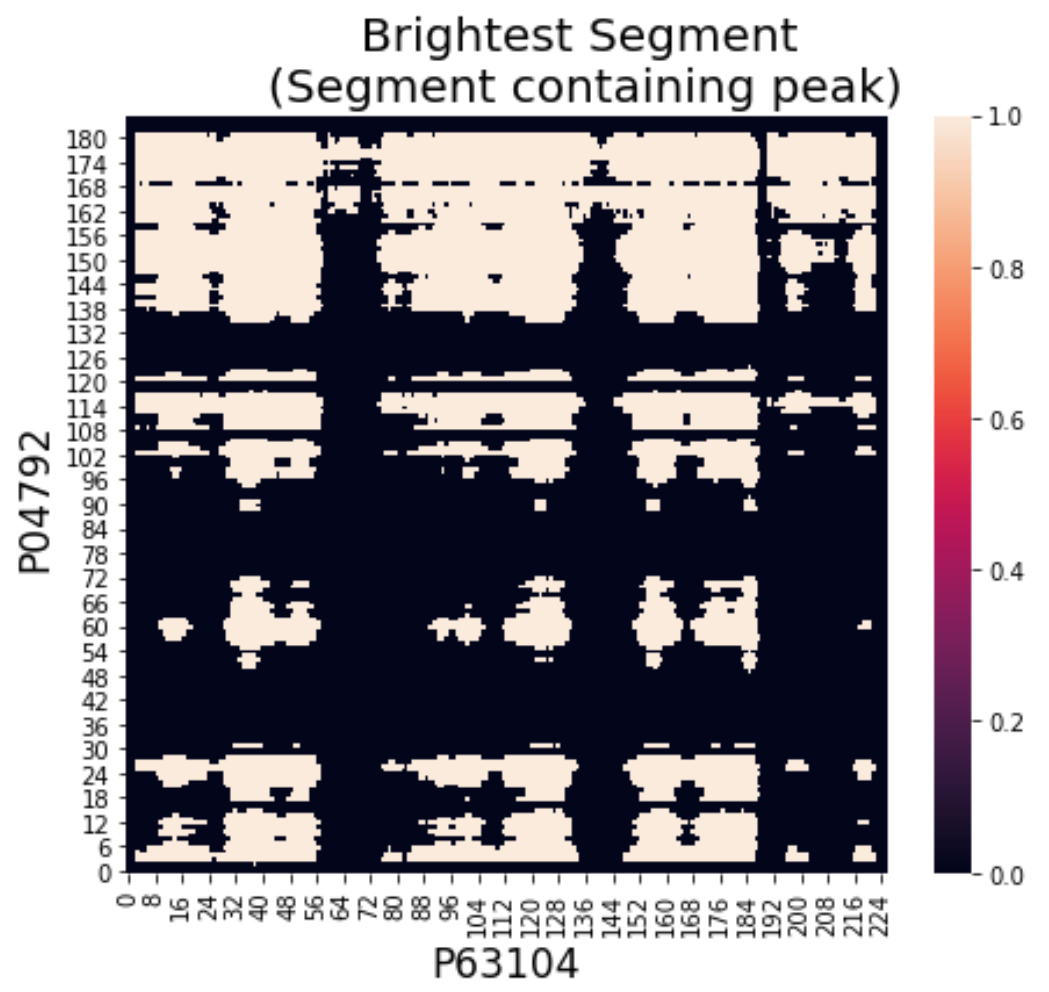

Figure 41: Brightest segment of the CLUSTER-NET output 


\section{Adding Gaussian blur to Cluster-Net Segment}

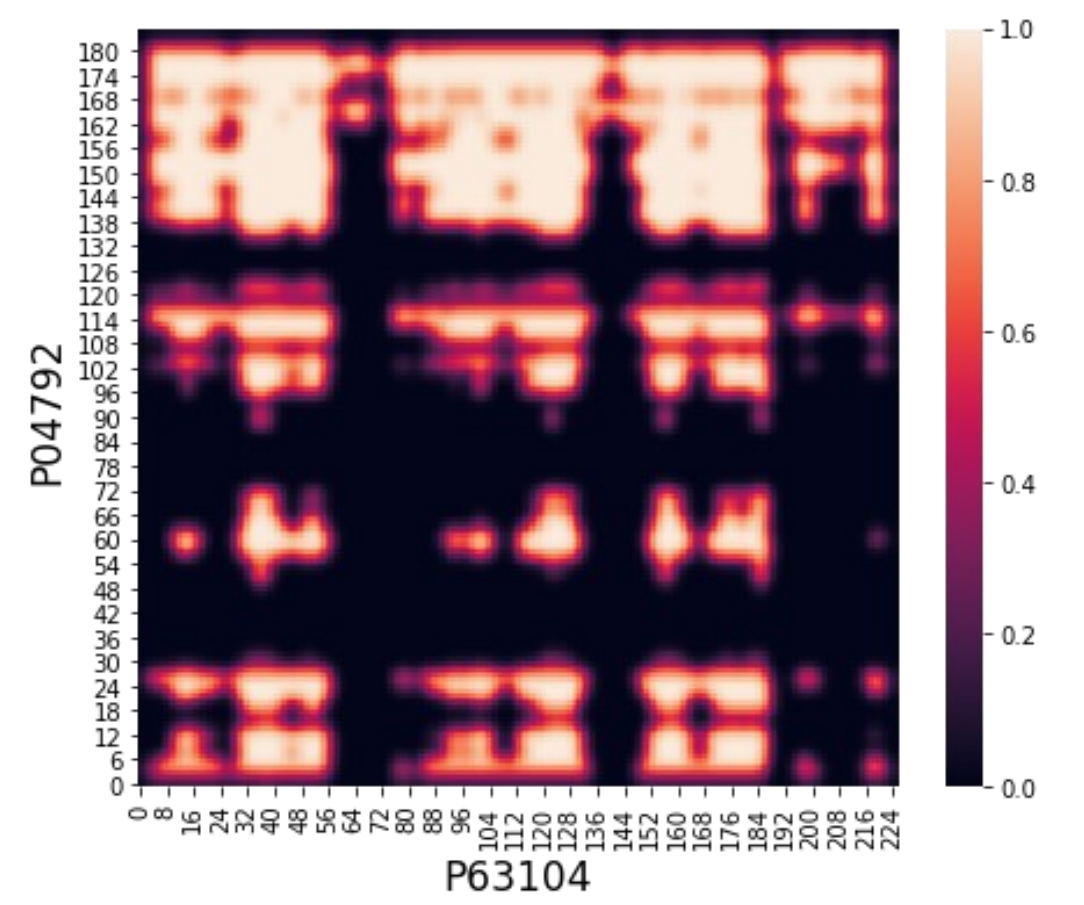

Figure 42: Reducing the overfitting of CLUSTER-NET by adding blur

Let us walk through this process with the example. The segment-2 (in Figure 40) contains the brightest pixel. Hence, we are calling it the brightest segment in Figure 41 . The output for segment-2 adding gaussian bluring is illustrated in Figure 42. Now, we can start working on segment-2. Additionally, segment-3 comes right after it in this order of brightness if segment-2 is exhausted and a total of 3 PPI-Sites are not detected yet. Now, within segment-2, we have a lot of separated continuous components. So once again, we rank them by their brightness. Finally, we calculate the minimum bounding rectangle enclosing top 3 ranked continuous-component. Figure 43 illustrates the bounding rectangle enclosing top 3 ranked continuous-components. These become the PPI-Sites predicted by CLUSTER-NET technique. A final comparison of these with the PPI-Site obtained in our lab verified validation data is illustrated at Figure 44 . 


\section{Minimum enclosing rectangle 1}

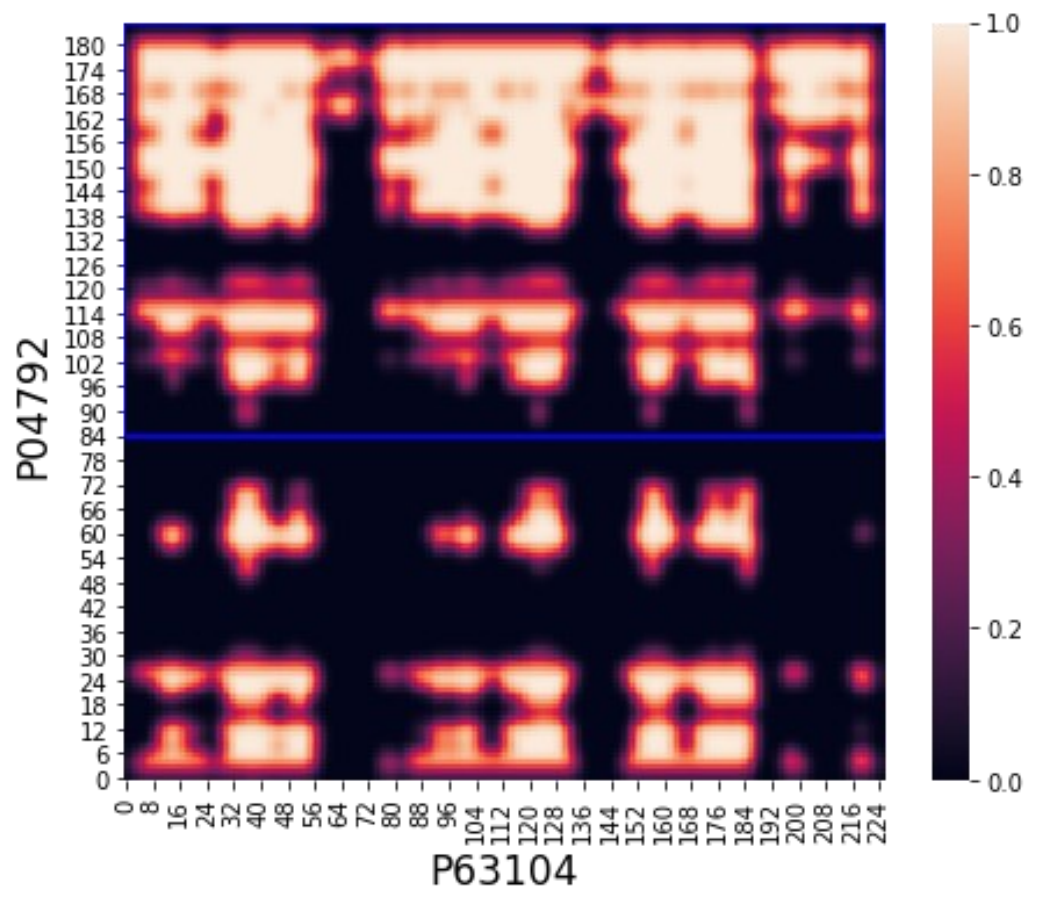

Minimum enclosing rectangle 2

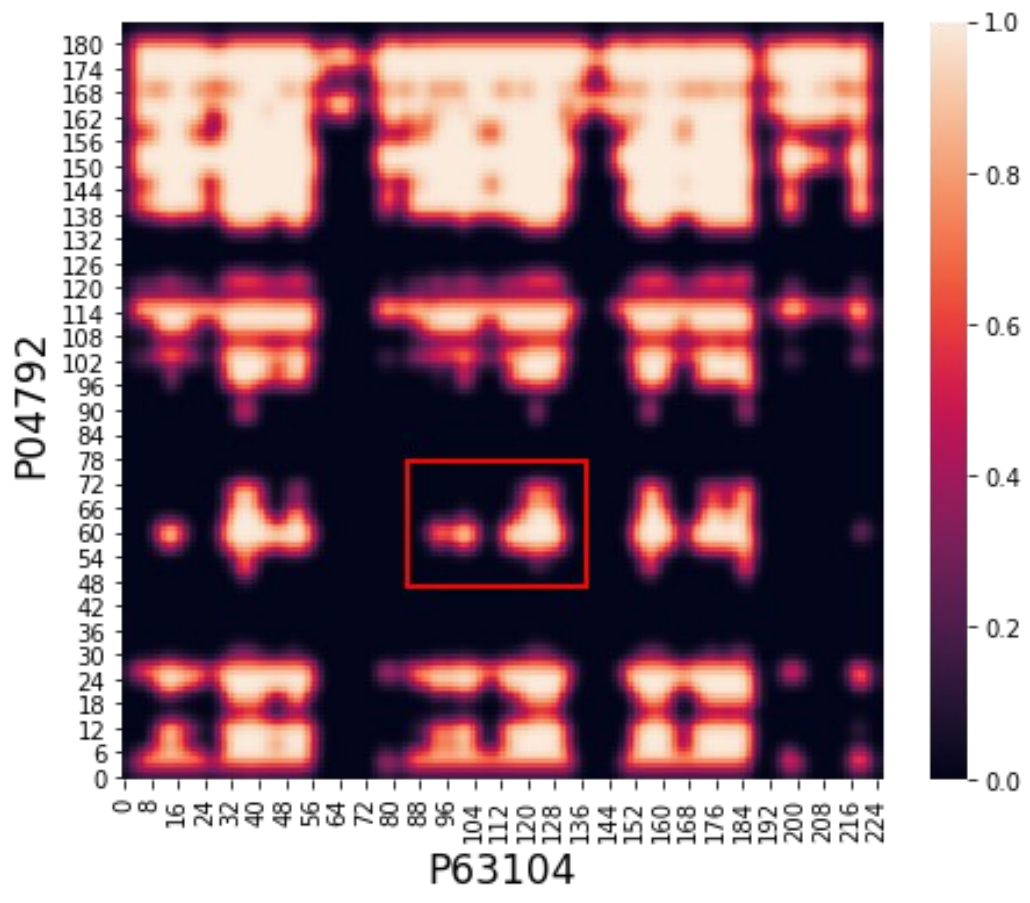




\section{Minimum enclosing rectangle 3}

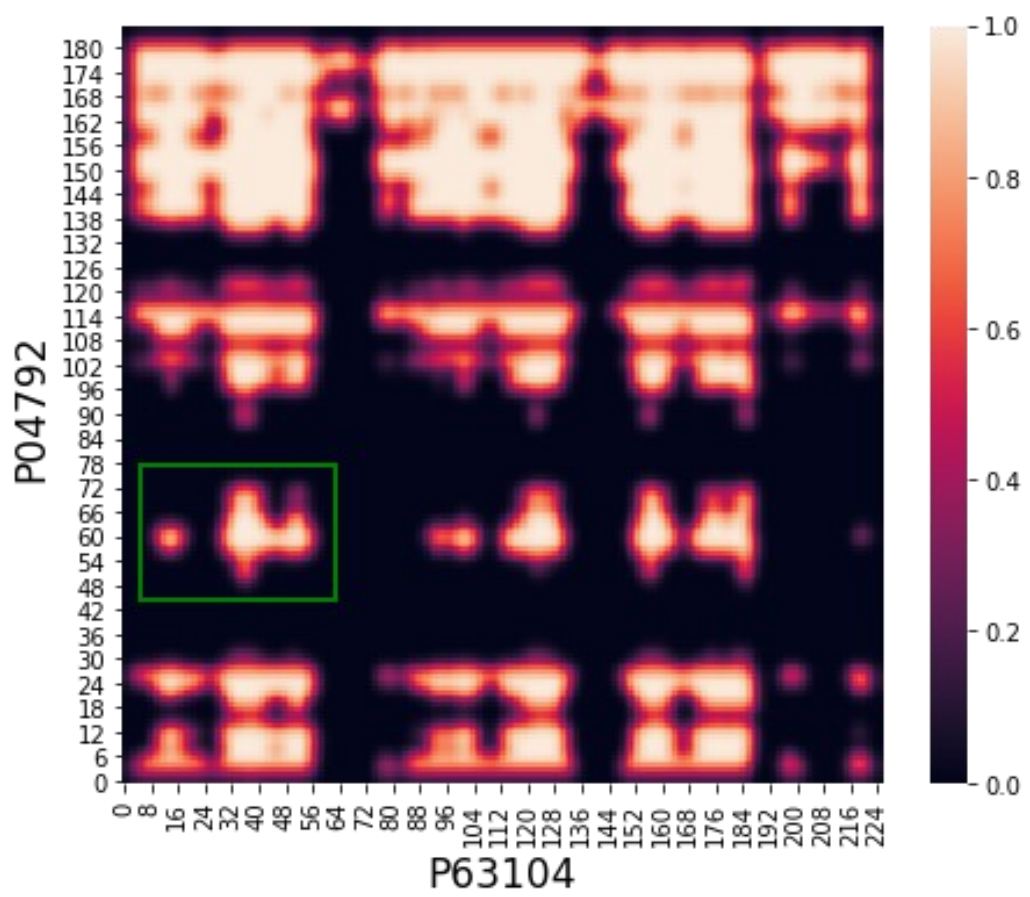

Figure 43: Minimum enclosing rectangle for top 3 ranked connected-components 


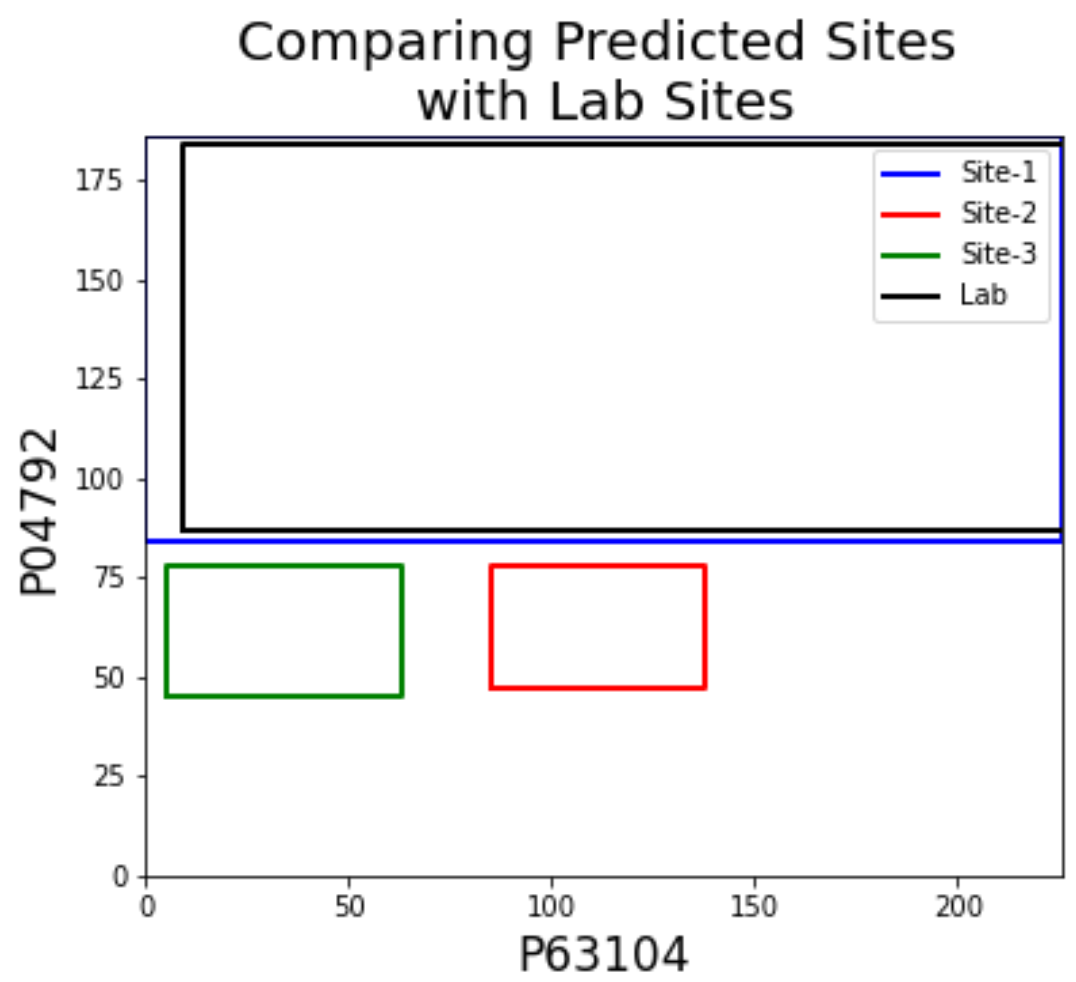

Figure 44: Comparing CLUSTER-NET prediction Sites with Lab verified PPI-Site

\subsubsection{Parameter Configuration}

Throughout various steps of CLUSTER-NET, we have a number of parameters that we can discuss. To recall the architecture of CLUSTER-NET, the configuration of first convolution layer is that it takes an input image, let's say with dimension 50x70x1, and applies $F$ convolutional kernels, each of size $K \times K$. It results into a total of $F$ number of features representing each pixel. The second convolutional layer has $C$ kernels of dimensions $1 \times 1$ each, making it a is a multiclass classifier. This classifies each pixel into a maximum of $C$ classes. Here performing a 3-way grid search across these parameters, similar to what we did in parameter optimization for BRIGHT-SPOT detection, means that we need to run the entire CLUSTER-NET training for each of the combinations of different values of these 
parameters. This results into enormous computational-runtime requirements. Therefore, inspired by empirical values used in original papers and after investigating various other CNN architecture default-parameters used for biomedical image processing [81], we concluded to use a total of 100 kernels of size $7 \times 7$ for the first layer, and a total of 50 kernels in the second layer, making it a 50 class classifier. Now it generates 100 features for each pixel and then use these features to classify each pixel in one of the 50 classes. We followed the traditional termination criterion which is to stop updating the neural network when it converges, meaning the error count (defined in earlier section) is significantly low. Now, to optimize the computational resources, we added another criterion to limit the number of learning iterations to a maximum to 500 steps.

\section{CLUSTER-NET paramter sweep on SW-landscapes}

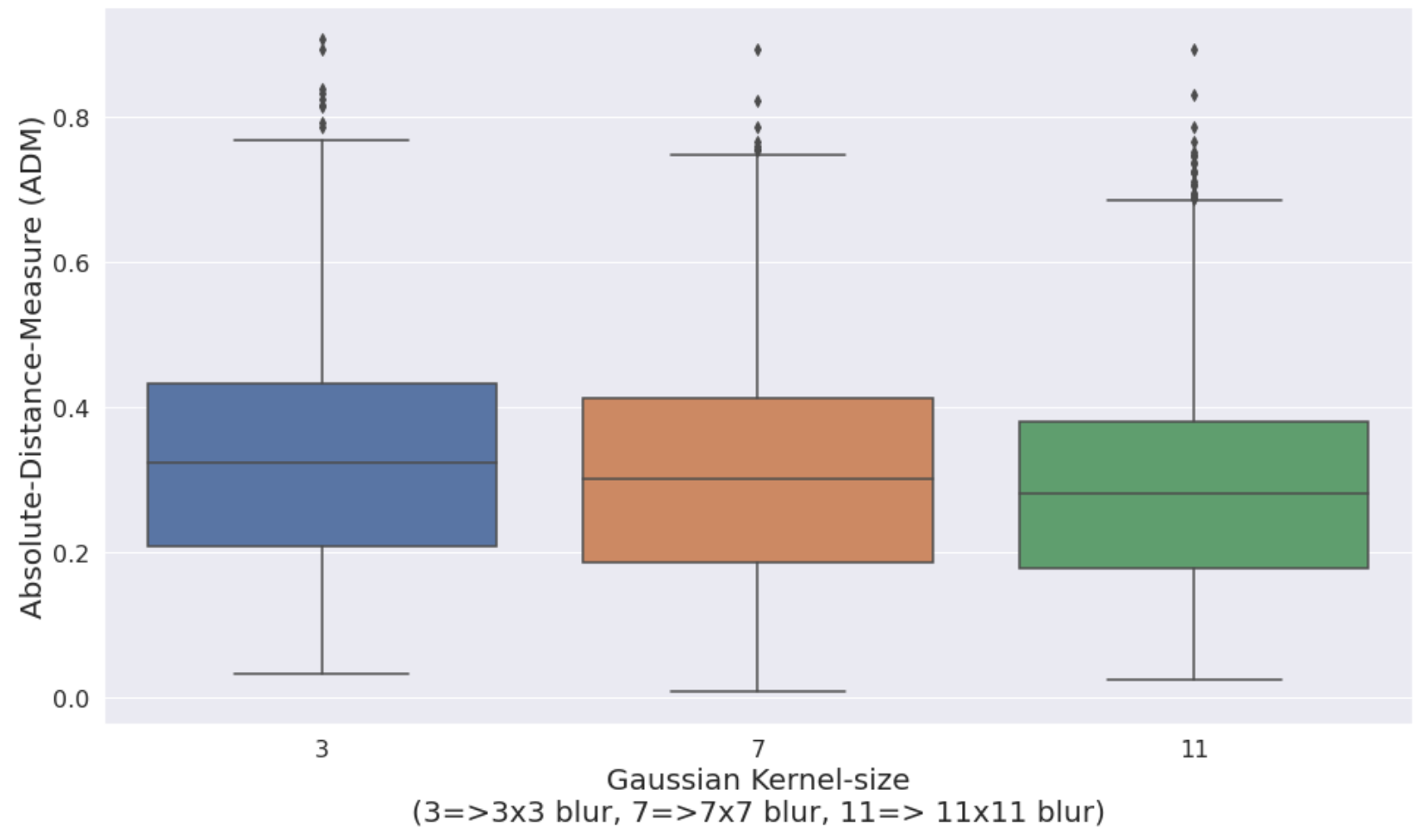

Figure 45: CLUSTER-NET performance for various kernel sizes 
Once the CLUSTER-NET predicts the classes with the above configurations, we have one last parameter left, the size of Gaussian Blur. Recall that it was added to smooth any overfitting that CLUSTER-NET might have perform. Figure 45 illustrates the experiments where we use one small kernel size (3x3), one medium kernel size (7x7) and one large kernel size (11x11). We used these values as the parameter-grid in Grid-Search CrossValidation [66] [67], like the previous algorithms. After plotting the final ADM distribution for protein-pairs, the lowest value is observed with the kernel of $11 \times 11$. CLUSTER-NET performance could improve further with increasing kernel size; however, due to enormous computational-runtime requirements we limited our parameter sweep to have $11 \times 11$ as the largest kernel in our experiments.

\subsubsection{WALK vs. CLUSTER-NET}

While comparing the ADM distribution of CLUSTER-NET configured with a mix of its best empirical and tested parameters, to the WALK algorithm with its best parameter, $30 \%$ of the peak, we see an improvement in prediction of PPI-sites as shown in the Figure 46. The box plot distribution visualization shows a decrease in ADM values for CLUSTER-NET. Statistical significance of differences will be discussed later in the thesis when discussing results of all the algorithms with each other. 


\section{Comparing WALK and CLUSTER-NET detection on SW-landscapes, with their best parameters}

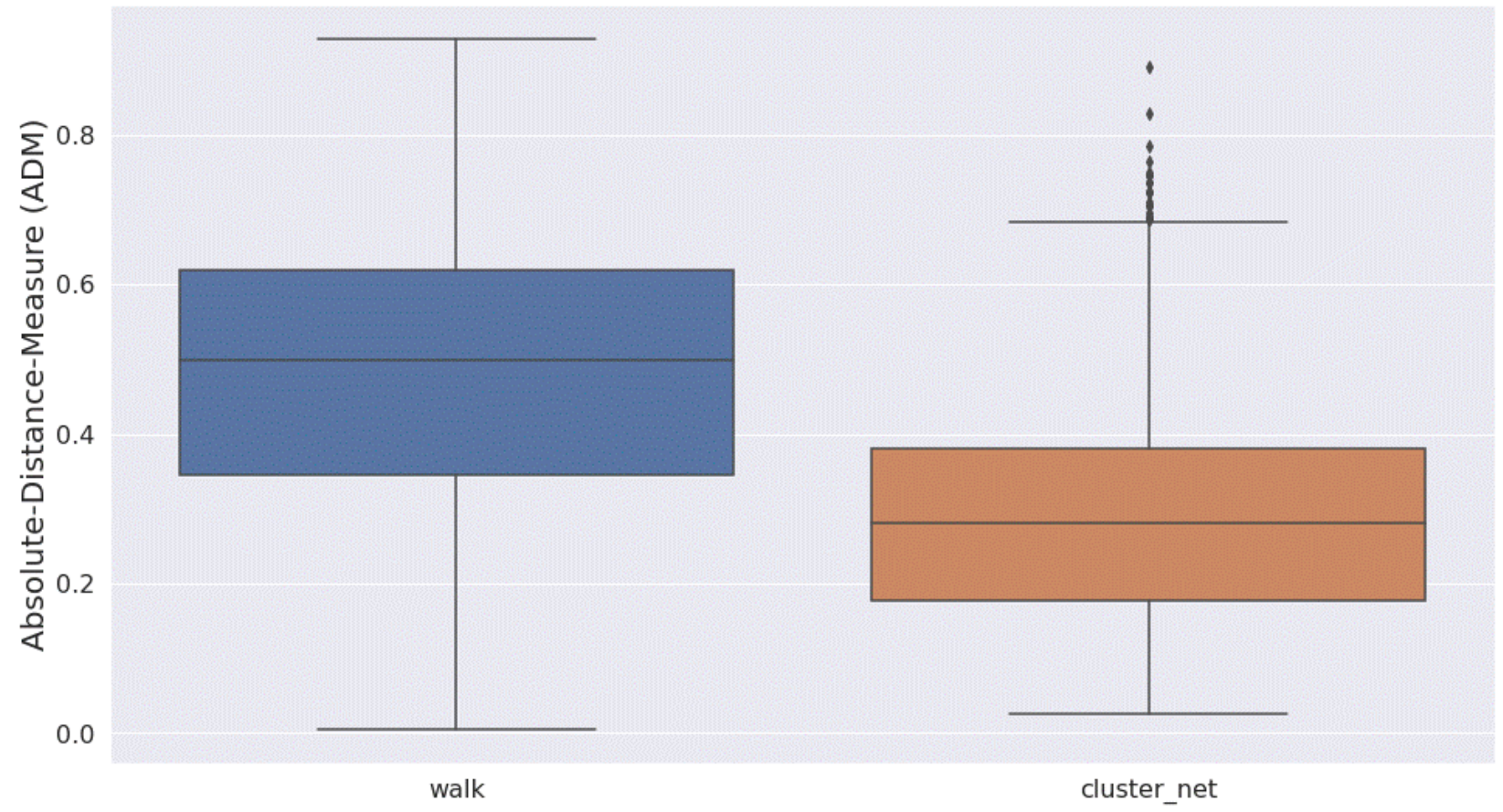

Figure 46: CLUSTER-NET vs. WALK

\subsection{Combined Results}

So far, in each of the sections we compared the performance of each algorithm to WALK on SW-landscapes. Each of the technique performs better when compared using box-plots representation. In this section, we not only compare all the techniques with each other but also perform statistical test to improve the confidence of the results. Lastly, we discuss another approach to compare the performance, named as Area Under the Curve (AUC).

\subsubsection{Box Plots}

In each of the sections thus far, we have compared individual new techniques with the retuned WALK algorithm. Figure 47 presents the wholistic comparison of all the new techniques with the current benchmark. As illustrated in Figure 47, we can see the box plot 
distribution (Y-axis) of all the new techniques compared with each other (X-axis) alongside WALK. Here we added two additional sites: Full-Sites and Peak-Sites. Full-Sites means the entire landscape is predicted as the PPI-Site, whereas the Peak-Site means just a single point, the peak, is predicted as the PPI-Site. We can consider these two as the absolute minimum baseline for PPI-Sites prediction. The distribution in Figure 47 suggests that even though WALK is performing better then Full-Sites and Peak-Sites, the rest of the three techniques are better than it. CLUSTER-NET is performing the best with the minimum ADM values. BRIGHT-SPOT detection seems to be performing slightly better than PANORAMA. Hence, statistical tests would be needed to draw the conclusion.

\section{Comparing WALK to all new algorithms, with their best parameters}

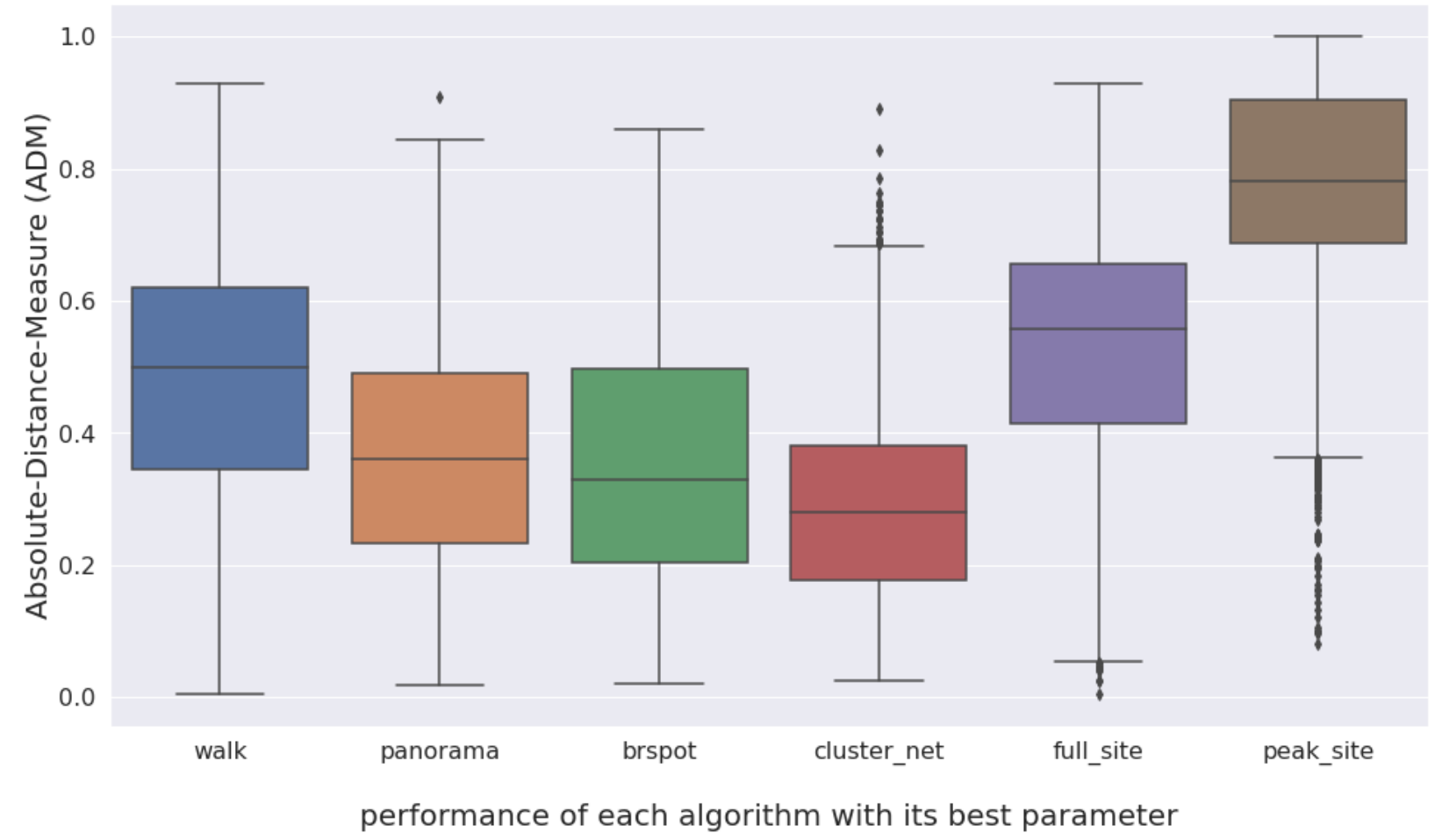

Figure 47: Box plot distribution of all new techniques compared to the WALK 


\subsubsection{Statistical Tests}

Since we are comparing ADM values for multiple algorithms, performing a test for Analysis of Variance (ANOVA test) would be most appropriate to begin with. If the ANOVA test finds at least some of the ADM values to be significantly different, we would need pairwise T-test to further decide which pairs of methods have significantly different performance.

\subsubsection{ANOVA test}

ANOVA tests as the name suggests analyses the variance with the group as well as across groups to check its null hypothesis, which is are the means of the group similar. Invalidating ANOVA test's hypothesis means that there exists at least one group that has statically significant difference from the rest. Hence after performing ANOVA test, if we need to further find out the differences between individual groups, we need to perform post-hoc tests. Now performing ANOVA test for all the four combined algorithms resulted in a significantly low $\mathrm{p}$-value (observed $\mathrm{p}<0.00001$ ), establishing that a statistically significant difference exists amongst the groups. Note that, here we performed a non-parametric ANOVA test referred as Kruskal-Wallis test [82].

Performing an "independent" T-test would involve comparing the means of ADM values of two Algorithms (two independent samples of scores) and testing for significant difference. This would be a two-sided test for the null hypothesis that ADM values of two Algorithms (2 independent samples) have identical average (expected) ADM values. However, the performance on any given protein pair is likely to be correlated between the two methods, 
as some pairs are likely to be easy, and some are likely to be difficult to predict the PPI-sites for any method. We therefore perform a "dependent" T-test where the difference in performance on each pair is calculated. This would be a two-sided test for the null hypothesis that the average difference in ADM values of two techniques (2 related samples) is zero. Invalidating the null hypothesis would mean that the expected ADM values are not same and hence the technique with lower mean ADM value is "statistically" better than the other.

Thus, we procced to perform the "dependent" T-test as this would be more meaningful measurement for comparing any two techniques.

\subsubsection{Performing Dependent T-test (post-hoc test)}

The most common dependent T-tests work best if the underlying data (the ADM distribution for a particular technique) is drawn from a normal distribution. Meaning, the more the data follow a normal distribution, the more reliable dependent T-test results will be. Since our data may not follow a normal distribution, we added another dependent Ttest called Wilcoxon Single-Rank Test [83]. Its null hypothesis states that the difference between the pairs follows a symmetric distribution around zero, without requiring any assumptions regarding the normality of the data. Invalidating its null hypothesis can be interpreted similarly to a t-test, where the technique with lower mean ADM value is "statistically" better than the other. The value Wilcoxon Single-Rank Test is bringing to the table is that its results are independent of the normality of the underlying data distribution. Table 1 illustrates the p-value for both the tests while comparing every technique with another. Here a small p-value (less than 0.01) means the null hypothesis is invalidated for 
that pair of technique and the one with smaller mean ADM is performing statistically better.

As illustrated in Figure 47, box-plot distribution clearly shows that CLUSTER-NET is performing the best. However, it does not clarify much between PANORAMA and BRIGHTSPOT detection. Now looking at Table 1, the p-value for both dependent T-test and Wilcoxon Single-Rank test is below the threshold for significance. Meaning that there is a statistically significant difference between PANORAMA and BRIGHT-SPOT detection. Since the mean value for BRIGHT-SPOT detection is lower, we can say that it performs significantly better than PANORAMA. Looking at the rest of the value in Table 1 confirms our observation from Figure 47, which is the order of performance is (best performing is put first): CLUSTER-NET, BRIGHT-SPOT detection, PANORAMA and WALK.

\begin{tabular}{|l|r|r|}
\hline & $\begin{array}{l}\text { dependent_t_test } \\
\text { for paired_samples }\end{array}$ & wilcoxon_t_test \\
\hline p-value & p-value \\
\hline walk - panorama & less than 0.00010 & less than 0.00010 \\
\hline walk - brspot & less than 0.00010 & less than 0.00010 \\
\hline walk - cluster_net & less than 0.00010 & less than 0.00010 \\
\hline panorama - brspot & 0.00616 & 0.00084 \\
\hline panorama - cluster_net & less than 0.00010 & less than 0.00010 \\
\hline brspot - cluster_net & less than 0.00010 & less than 0.00010 \\
\hline
\end{tabular}

Table 1: T-test for statistical significance 


\subsubsection{AUC : Area Under the Curve}

Performing T-tests combined with box-plot distribution gives us the conclusive results of comparison of techniques. However, the trend of how well a technique is performing can be captured through another means: the Area Under the Curve (AUC). In traditional supervised Machine Learning approaches, we plot the curve of Precision and Recall (PRcurve) to identify the performance as the decision threshold is varied and to look for trends that can help for further improvements. For example, a steep slope in the beginning of a PR-curve is considered very good whereas a flatness is less desirable. We have plotted a similar curve, as illustrated in Figure 48, where the X-axis represents the ADM value and the $y$-axis represents number of protein-pairs below that ADM value. For example, the best 500 protein-pairs of CLUSTER-NET (represented as green) are below 0.18 ADM value whereas best 500 protein-pairs of Peak-Site (represented as black) are below 0.7 ADM value. Hence, we can say that CLUSTER-NET is performing better that Peak-Site for their first 500 protein-pairs. Here also, a high steep in the beginning means more and more protein-pairs for that technique have a small ADM value, and hence represents a very good technique. Whereas a flatness in the beginning represents a low number of protein-pairs have small ADM values representing a low performing technique.

Figure 48 illustrates that apart from the very beginning (couple of protein-pairs), the trend is quite consistent, which is CLUSTER-NET (in green), followed by BRIGHT-SPOT detection (in orange), followed by PANORAMA (in blue) and lastly WALK (in red). Both Full-Sites (in magenta) and Peak-Sites (in black) are the lowest performing ones. This not only matches 
with the earlier results but also affirms that BRIGT-SPOT detection and PANORAM are performing close by.

AUC for various algorithms running on SW-landscapes of human protein-pairs

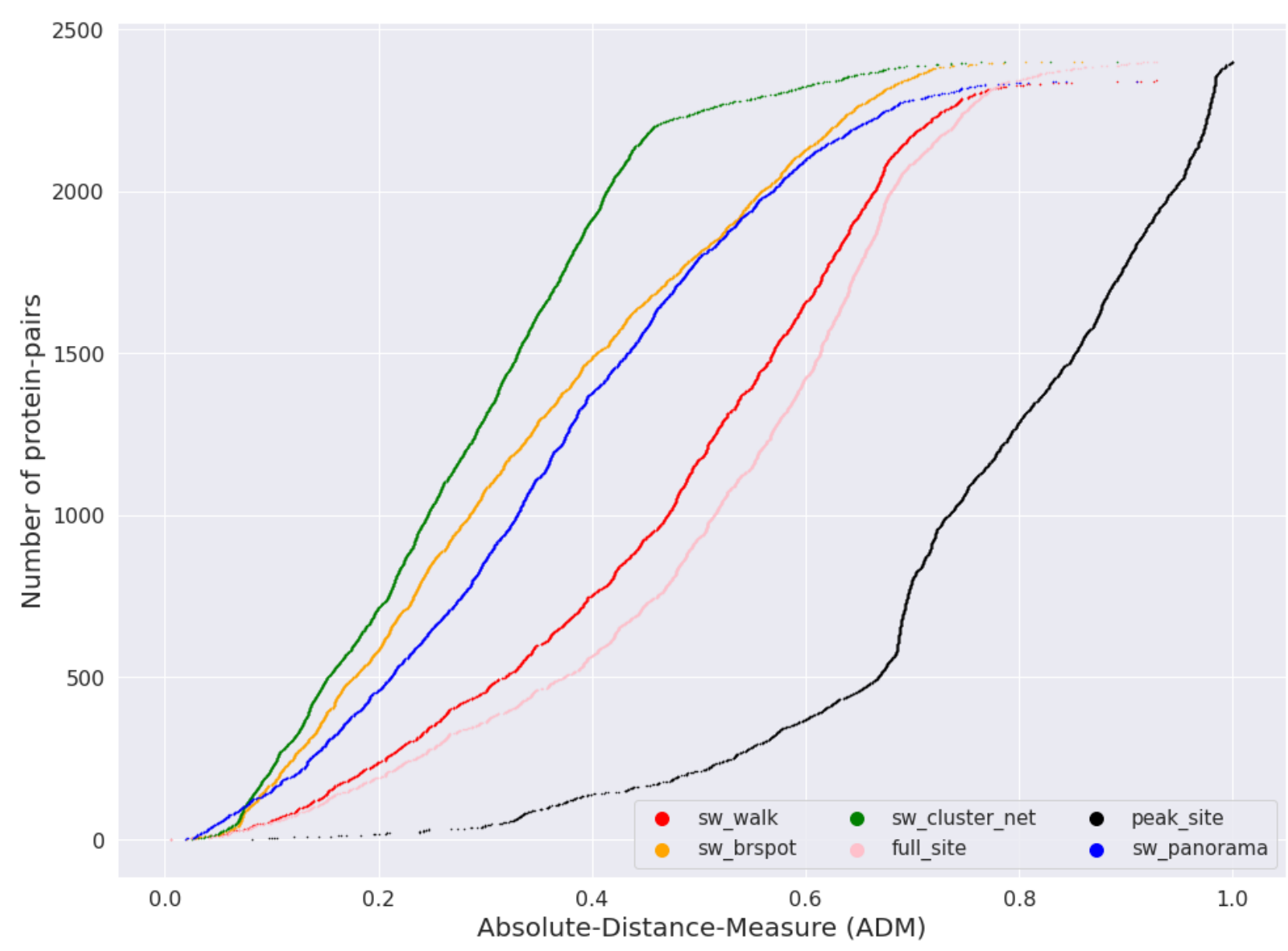

Figure 48: AUC for WALK and all new techniques

\subsection{Infrastructure details}

All the new methods introduced in this chapter were developed in python framework. We used CV2 library functions [71] for most of the image preprocessing steps like adding Gaussian blur, applying threshold, and many more. Additionally, we used PyTorch [84] for 
setting up the neural network configuration and parameters. All the experiments, training and performance evaluations, were performed on AWS infrastructure with m5.24xlarge specifications [65], which consisted of $64 \mathrm{vCPUs}$ and 256 GiB memory.

Runtime for each of the algorithm was also measured on the above setup. For PANORAMA, the run time to predict the PPI-sites for all 2500 human protein-pairs, on 64 vCPUs was about 200 seconds. For BRIGHT-SPOT detection, the runtime for same number of proteinpairs on 64 vCPUs was about 4200 seconds. Lastly, for CLUSTER-NET, this run time was about 20,000 seconds. We excluded the Grid-Search Cross-Validation from this run-time, since, for each algorithm, it would be multiple of its runtime with for every grid configuration. Adding more CPUs to existing infrastructure capacity would lead to smaller runtime for PANORAMA and BRIGHT-SPOT detection. However, for CLUSTER-NET, adding extra GPU would be beneficial to boost the runtime.

\subsection{Conclusion}

In this chapter we introduced new PPI-site detection techniques, compared those to WALK, and finally performed a comprehensive comparison. We used human protein-pairs dataset to fine tune our models and established the results. In next chapter, we use yeast protein-pairs to validate our findings and verify the robustness of our new techniques. 


\section{Validation of new PPI-Site detection techniques on Yeast}

In the previous chapters, we performed experiments for PPI-Site predictions for various techniques and established their performance. These experiments were conducted for Human protein-pairs. In this chapter, we validate our results and the performance of various techniques for protein-pairs in Yeast. We discuss the collection of datasets for Yeast, followed by PPI-Sites prediction for all the techniques and lastly compare their performance.

\subsection{Dataset for Yeast}

The most recent list of yeast protein pairs known to physically interact was extracted from BioGrid. Where, like human protein-pairs, we only considered physical interactions, of which there were 17,730 interacting protein pairs. For each protein pairs, UniProt is used to get the sequences for the individual proteins. A total of 4,056 yeast protein sequences are available in UniProt. PIPE was used to generate SW-landscapes for each protein pair. These SW-landscapes are used as input to all the new PPI-site prediction algorithms we described in previous chapter, along with original PPI-Sites detection method WALK.

To evaluate the accuracies of the predicted PPI sites, we again use the lab-verified database of 3D Interaction Domains (3did). After processing the custom file provided by 3did, we obtained 1,088 pairs of domain-domain interactions. Pfam's REST-API was used to retrieve and categorise the available 17,730 interacting protein-pairs into three categories: 
1. No-domain interaction-pairs (16,468 pairs). For these interacting protein pairs, no interacting domains could be identified.

2. Single-domain interaction-pairs (460 pairs). Each Single-domain interaction-pair had one domain in each of the protein that was known to interact.

3. Multi-domain interaction-pairs (802 pairs). Multi-domain interaction-pairs means that the interacting protein-pairs had multiple domains in at least one of the two proteins of the interacting protein-pair.

Since the experiments on human protein-pairs were performed only on single-domain interaction pairs, here also we focused only on 460 single-domain interaction-pairs. These are treated to as the lab-verified sites (a.k.a. ground truth PPI interaction site dataset) for yeast.

\subsection{Comparing various techniques on Yeast dataset}

In previous chapters, we introduced various techniques to detect PPI interaction sites and performed their optimization by using protein-pairs of human species. In this section we compare the performance of these techniques, with their optimized hyperparameters, on yeast PPI. We begin with the box-plot comparisons of ADM distributions, followed by Ttests to check the statistical significance of the difference. Lastly, we plot the AUC to observe the performance trend. 


\subsubsection{Box Plots comparison}

Recall that the box-plot comparison illustrates the ADM distribution of protein-pairs for all the techniques. Here, box-plot being closer to zero indicates it to be a better technique over others. Figure 47 illustrates the box-plot for protein-pairs of human where we used the optimized parameters for each algorithm. The choices for the optimized parameters for each algorithm were discussed previously in their respective sections. In this section, we used these same parameter values and calculate the ADM for all yeast protein pairs. Figure 49 illustrates the box-plot distribution for each method. Both the BRIGHT-SPOT detection and CLUSTER-NET seems to be performing better than PANORAMA and WALK. However, a statistical test is required to verify the difference of performance between BRIGHT-SPOT detection and CLUSTER-NET, as well as between PANORAMA and WALK. Lastly, we can see that each algorithm shows a better performance in comparison to the baseline approaches - Full-Site, Peak-Site.

These results strongly suggest that the parameter optimization of each algorithm performed for human protein-pairs, also works on yeast protein-pairs. 


\section{Running all algorithms with their best parameters, on SW-landscapes for yeast}

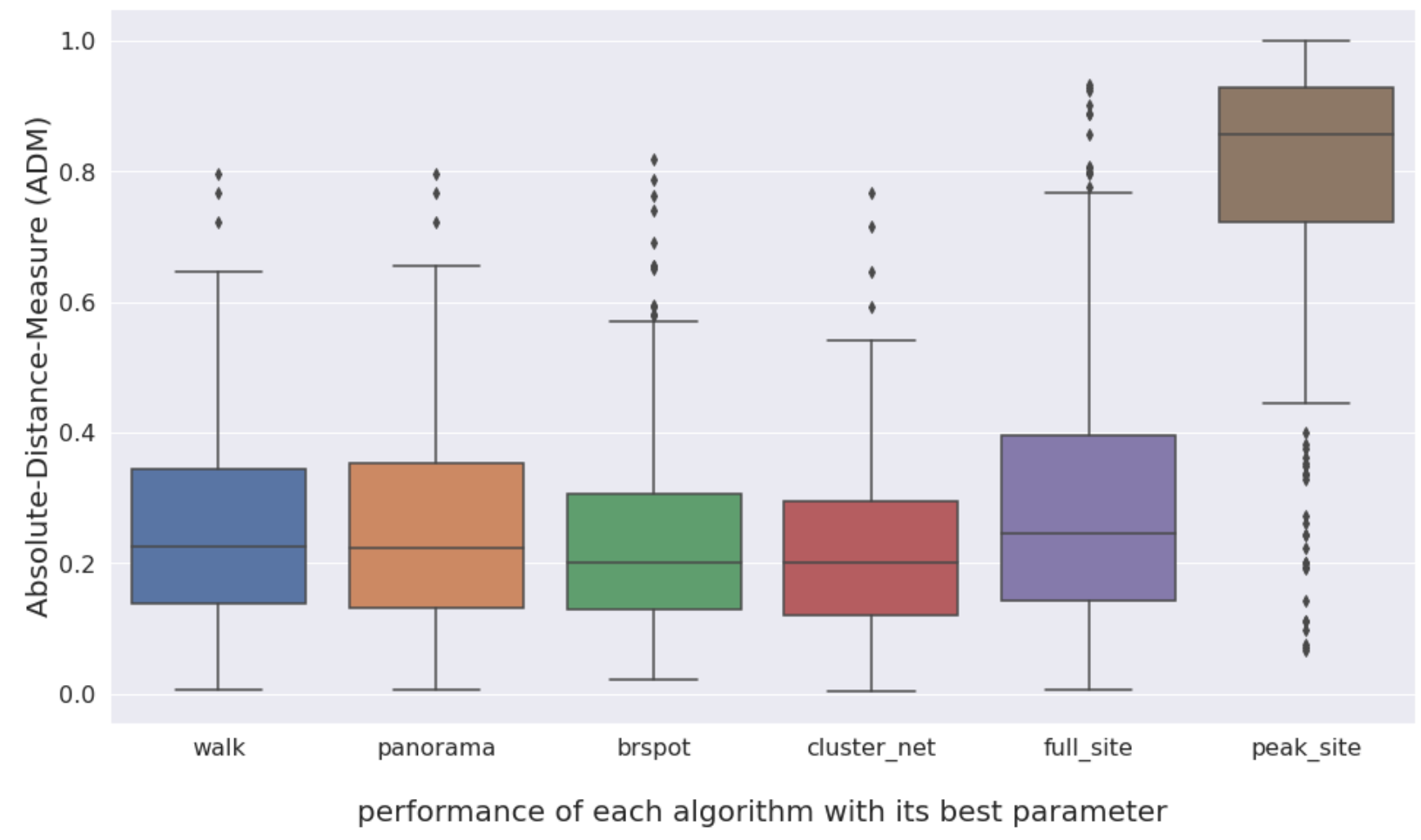

Figure 49: Box-plot distribution of yeast protein-pairs for all techniques with optimized parameters

\subsubsection{Statistical Tests}

We first performed non-parametric ANOVA test using the Kruskal-Wallis method to compare ADM values of all the algorithms for yeast protein-pairs. The p-value was observed to be 0.00358, making it sufficiently small to reject null hypothesis and establishing that there is a statistically significant difference amongst the groups. After this, as a part of post-hoc tests, we compared the difference of performance for each pair of algorithms with two statistical test - Dependent T-test and Wilcoxon T-test. Results from both tests for yeast protein-pairs are included in Table 2. 


\begin{tabular}{|l|r|r|}
\hline & $\begin{array}{l}\text { dependent_t_test } \\
\text { for paired_samples } \\
\text { p-value }\end{array}$ & \multicolumn{1}{l|}{$\begin{array}{l}\text { wilcoxon_t_test } \\
\text { p-value }\end{array}$} \\
\hline walk - panorama & 0.98951 & 0.00742 \\
\hline walk - brspot & 0.2241 & less than 0.00010 \\
\hline walk - cluster_net & less than 0.00010 & less than 0.00010 \\
\hline panorama - brspot & 0.24596 & 0.00057 \\
\hline panorama - cluster_net & less than 0.00010 & less than 0.0001 \\
\hline brspot - cluster_net & 0.01907 & 0.46623 \\
\hline
\end{tabular}

Table 2: Performing T-tests on yeast

As discussed in previous section, the box-plot distribution in Figure 49 shows that CLUSTER-NET and BRIGHT-SPOT detection are performing better than PANORAMA and WALK. Table 2 illustrates that at least one of the tests has significantly low p-value when BRIGHT-SPOT detection is compared to CLUSTER-NET, as well as when WALK is compared to PANORAMA. As described previously in the case of low p-value, the null hypothesis is considered rejected and the algorithm with lower mean ADM value is considered to be performing better. This concludes the order of performance, which is (best performing is put first): CLUSTER-NET, BRIGHT-SPOT detection, PANORAMA and WALK.

\subsubsection{Area Under the Curve Performance}

We discussed in the previous chapter that the AUC provides a trend of performance of the algorithm. Recall that it behaves similar to a PR-curve, in that a steep incline in the beginning implies that the algorithm is performing better, whereas flatness in the beginning indicates a low-performing algorithm. Figure 50 illustrates the AUC for all the algorithms including the baseline (Full-Site and Peak-Site). 
yeast : AUC for various techniques for FPR $<0.5$

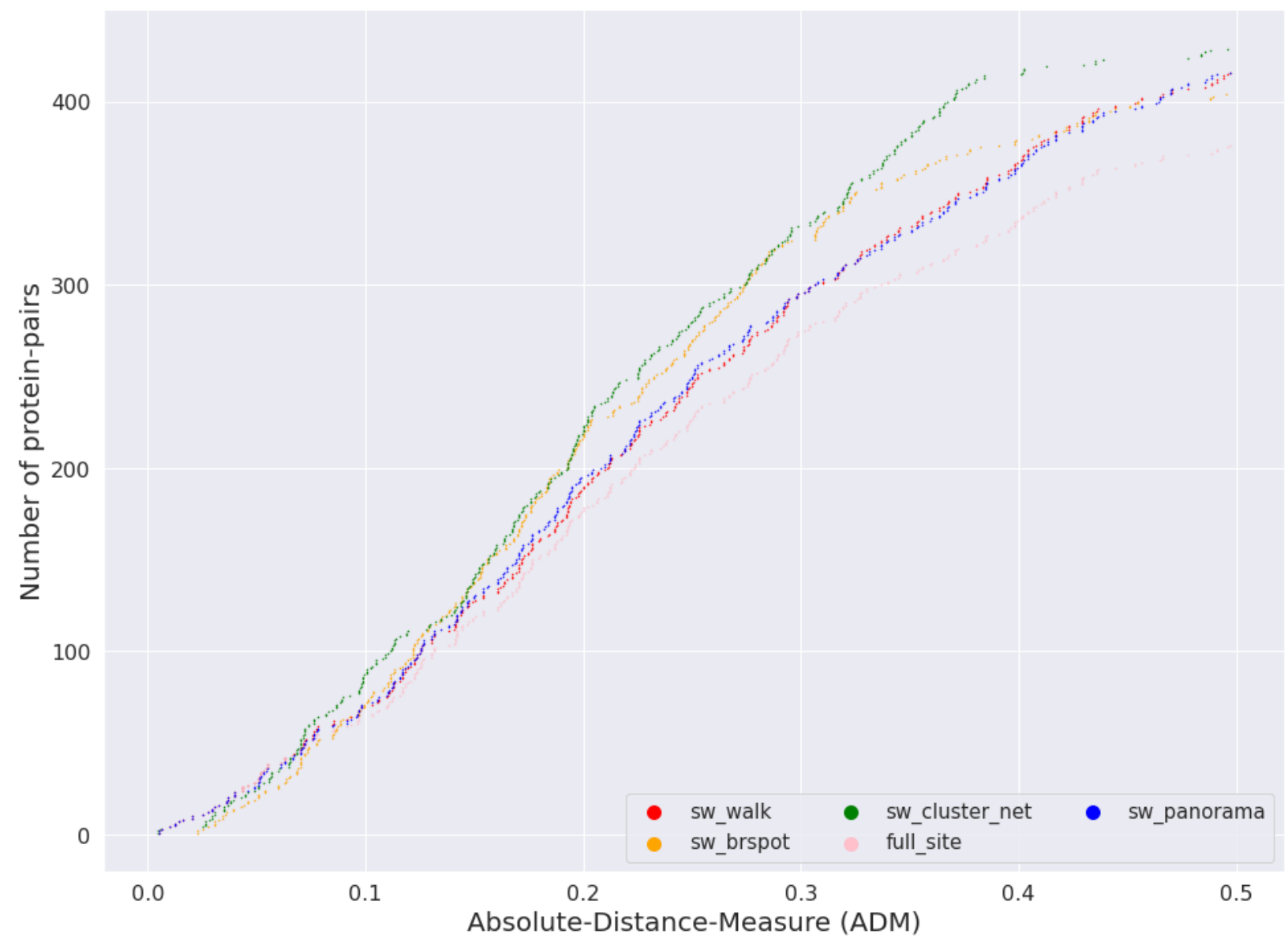

Figure 50: AUC for yeast

The trend illustrated in the AUC also shows that CLUSTER-NET (in green) and BRIGHT-SPOT detection (in yellow) are performing better than PANORAMA (in blue) and WALK (in red). Examining the trend for DM values $<0.5$, as illustrated in Figure 51, reveals that CLUSTER-NET performs better than BRIGHT-SPOT detection for all the protein-pair whereas PANORAMA performs better than WALK for most of the protein-pairs. These trends matched with the above results, affirming the effectiveness of algorithms in yeast protein-pairs are same as they are for human protein-pairs. 


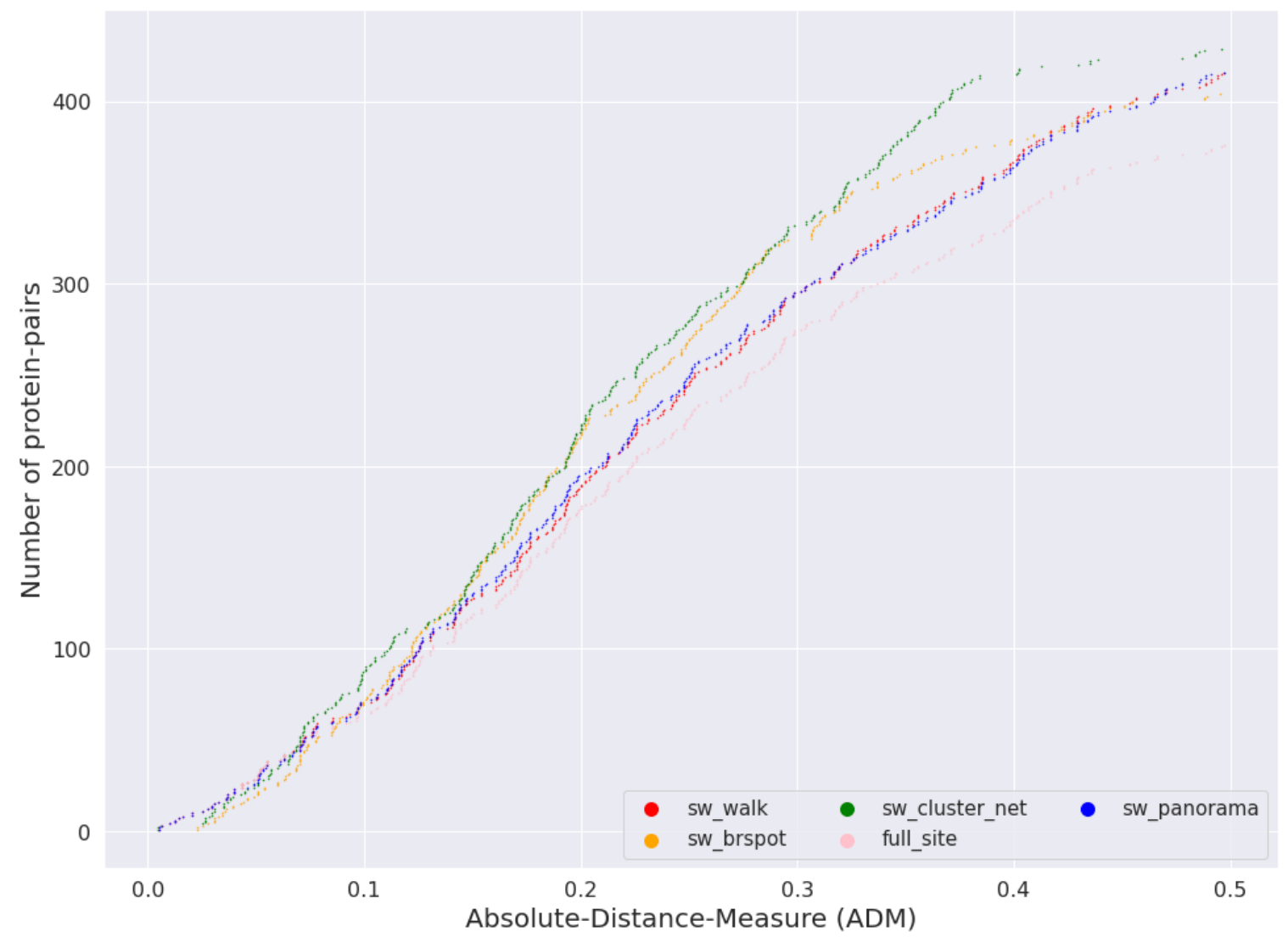

Figure 51: Zooming into AUC for yeast for FPR $<0.5$

\subsection{Conclusion}

In previous chapters, we developed various new algorithms and optimized their parameters using human protein-pairs. In this chapter, we used these optimized algorithms to predict PPI-Sites on yeast protein-pairs. Running these optimized algorithms on human protein-pairs concluded that CLUSTER-NET performs the best, followed by BRIGHT-SPOT detection, followed by PANORAMA and lastly, WALK. The results discussed in this chapter also revealed that CLUSTER-NET performs the best for yeast and rest of the algorithms follow the same order, confirming that these optimized parameters also work successfully beyond one species. 


\section{Thesis Summary and Future Recommendations}

\subsection{Conclusions}

Over the course of this work, we demonstrated that the original Distance Measure (DM) metric failed to capture the true performance of PPI site predictions. When the prediction PPI-site is entirely contained within the lab verified PPI-site, then it fails to penalize underprediction. We introduced a new metric, the Absolute Distance Measure (ADM), to overcome this and used it to evaluate the performance of all the algorithms. We built a new high-quality dataset for human protein pairs, along with their lab verified PPI-Sites. We used this dataset to re-tune the WALK algorithm, optimizing for the best value of the parameter "threshold" - some percentage of the peak height. Evaluation using the ADM over the new dataset established that a threshold of $30 \%$ of peak height provided improved performance relatively to the previously used threshold of $50 \%$, when run against the original PIPE-Score landscapes. However, with SW-landscapes, the WALK algorithm is incapable of effectively identifying the PPI-Sites, even after performing the hyper parameter tuning.

After concluding the poor performance of WALK for predicting PPI-sites on SW-landscapes, we introduced three new algorithms for PPI-site prediction - PANORAMA (very similar to WALK), BRIGHT-SPOT detection (a computer vision approach), and CLUSTER-NET (a deeplearning method). PANORAMA has the hyper parameter "threshold", similar as walk, for which the optimized value was obtained as $50 \%$ of the peak. With this parameter, it performs better than WALK on SW-landscapes. 
BRIGHT-SPOT detection has two parameters - size of the Gaussian Kernel and threshold. A two-way grid search cross-validation reveals that a threshold value of $30 \%$ of peak height and a kernel size of 7x7 are best. With these values, it performs better than WALK on SWlandscapes. Finally, CLUSTER-NET uses a CNN with 2 convolution layers combined with batch normalization and an arg-max layer. The first convolution layer has 100 kernels of size $7 \times 7$ each, and the second convolution layer has 50 kernels making it a 50-class classifier. A final $11 \times 11$ kernel is used on the CLUSTER-NET output to find the minimum enclosing rectangles as the predicted PPI-sites. This also displayed an improved performance on SW-landscape when compared with WALK.

In the end, we illustrated the performance of all the algorithms using a box-plot comparison, followed by statistical tests and Area Under the Curve (AUC) analysis. The results on human protein-pairs concluded the order of performance, in decreasing order of performance: CLUSTER-NET, BRIGHT-SPOT detection, PANORAMA, and WALK. Which was then confirmed in a separate yeast dataset using the same prediction models and optimized parameters, demonstrating generalization across dataset and species.

\subsection{Summary of contributions}

In Chapter 3, we demonstrated a new data extraction pipeline capable of extracting high quality lab verified PPI dataset. This pipeline is used to extract 2532 Single-domain interaction-pairs, 5321 Multi-domain interaction Pairs, and 58231 No-domain interaction- 
pairs. We only focused on 2532 Single-domain interaction-pairs for the rest of the research work. We introduced an enhanced distance measure ADM which, unlike its predecessor, penalizes underpredictions of PPI sites. We introduced the optimization needed in the inherent PIPE-Sites detection technique, WALK, when predicting PPI-Sites on the landscapes of these new high-quality datasets measured on ADM. This concluded that WALK works best with parameter value 0.3 , meaning when threshold is set as $30 \%$ of the peak height. However, WALK does not perform well on SW-landscapes, which motivated us to consider alternative techniques.

In Chapter 4, we introduced various different technique to detect better PIPE-Sites than WALK. We started with PANORAMA which is a very similar approach to WALK and differs in one major aspect which is that it looks for more amino acids than just at one location. However, this technique performed similarly to WALK when it came to predicting PIPESites for SW-landscapes. We introduced a second technique called BRIGHT-SPOT detection. It treated input SW-landscape as an image and performed classical images processing to detect the bright spots in the landscape. Using the optimized parameter of $30 \%$ and a $7 x 7$ kernel size, yielded improved results relative to PIPE-Sites. Seeing that image processing led to improved results, it inspired us to look for more state-of-the-art image processing techniques that led to our final method, CLUSTER-NET, to detect PPI-Sites. This unsupervised deep learning approach used CNN and a pixel segmentation technique that uses iterative clustering to generate labels which were used to train the CNN parameters. With this architecture and a kernel of size $11 \times 11$ it provides the best performance of all methods in predicting PPI-sites. 


\subsection{Recommendations for future work}

CLUSTER-NET is inspired by recently proposed unsupervised deep learning techniques. Since deep learning is advancing rapidly, we can expect that advances in image analysis methods will likely also be applicable to the PPI-site prediction problem. Lastly, the methods developed here should also be validated on additional species, including interspecies (e.g. host-pathogen) PPI interaction site data. It is possible that methods must be adapted for such inter-species prediction tasks. Hyperparameter tuning for each species may be useful before actual deployment of our algorithm. Furthermore, future validations could be done on more independent datasets to increase the confidence in the validity of the methods.

In the PPI-site prediction problem, we balance two types of errors in our problem - falsepositives, where the prediction site is larger than actual site (over-prediction), and falsenegative, where prediction of site falls shorter than actual site (under-prediction). The cost of both types of errors differs and depends on the application., For example, if PPI-site prediction is used for protein engineering, a high false-positive rate would undermine months of wet-lab validation efforts, whereas high rate of false-negatives may make it impossible to develop an effective vaccine or drug. Therefore, it is recommended to balance these two as most appropriate for the target application.

Image augmentation techniques, such as reversal or flipping the image landscapes, could be used to increase the potential training data size. However, this should be used with caution since we do not see reversed domain in natural proteins. This is due to the fact that AA 
sequences in proteins are directional. Landscapes which are input to our methods, are generated through PIPE, which uses a window of $20 \mathrm{AA}$ in any protein sequence. Changing this size may have an impact on input landscape but is outside the scope of this work. 


\section{References}

[1] J. Zahiri, J. Bozorgmehr, and A. Masoudi-Nejad, "Computational Prediction of ProteinProtein Interaction Networks: Algorithms and Resources," Current Genomics, vol. 14, no. 6,2013 , doi: $10.2174 / 1389202911314060004$.

[2] F. H. Stephenson, Calculations for Molecular Biology and Biotechnology: Third Edition. Elsevier Inc., 2016.

[3] M. Kotlyar, C. Pastrello, A. E. M. Rossos, and I. Jurisica, "Protein-protein interaction databases," in Encyclopedia of Bioinformatics and Computational Biology: $A B C$ of Bioinformatics, vol. 1-3, 2018.

[4] M. Shatnawi, "Review of Recent Protein-Protein Interaction Techniques," in Emerging Trends in Computational Biology, Bioinformatics, and Systems Biology: Algorithms and Software Tools, 2015.

[5] T. Sun, B. Zhou, L. Lai, and J. Pei, "Sequence-based prediction of protein protein interaction using a deep-learning algorithm," BMC Bioinformatics, vol. 18, no. 1, 2017, doi: 10.1186/s12859-017-1700-2.

[6] K. Dick and J. R. Green, "Reciprocal Perspective for Improved Protein-Protein Interaction Prediction," Scientific Reports, vol. 8, no. 1, 2018, doi: 10.1038/s41598018-30044-1.

[7] A. Amos-Binks et al., "Binding Site Prediction for Protein-Protein Interactions and Novel Motif Discovery using Re-occurring Polypeptide Sequences," BMC Bioinformatics, vol. 12, 2011, doi: 10.1186/1471-2105-12-225.

[8] D. Sarkar and S. Saha, "Machine-learning techniques for the prediction of proteinprotein interactions," Journal of Biosciences, vol. 44, no. 4. 2019, doi: 10.1007/s12038-019-9909-z. 
[9] S. Hashemifar, B. Neyshabur, A. A. Khan, and J. Xu, "Predicting protein-protein interactions through sequence-based deep learning," in Bioinformatics, 2018, vol. 34, no. 17 , doi: $10.1093 /$ bioinformatics/bty573.

[10] S. Pitre et al., "PIPE: A protein-protein interaction prediction engine based on the reoccurring short polypeptide sequences between known interacting protein pairs," BMC Bioinformatics, vol. 7, 2006, doi: 10.1186/1471-2105-7-365.

[11] T. Sevimoglu and K. Y. Arga, "The role of protein interaction networks in systems biomedicine," Computational and Structural Biotechnology Journal, vol. 11, no. 18. Elsevier, pp. 22-27, Aug. 01, 2014, doi: 10.1016/j.csbj.2014.08.008.

[12] A. Schoenrock et al., "Efficient prediction of human protein-protein interactions at a global scale," BMC Bioinformatics, vol. 15, no. 1, 2014, doi: 10.1186/s12859-0140383-1.

[13] B. D. Barnes, "Accelerated Transfer Learning for Protein-Protein Interaction Prediction," Jan. 2018. doi: https://doi.org/10.22215/etd/2018-12707.

[14] D. Szklarczyk et al., "STRING v10: Protein-protein interaction networks, integrated over the tree of life," Nucleic Acids Research, vol. 43, no. D1, 2015, doi: 10.1093/nar/gku1003.

[15] R. Jothi, P. F. Cherukuri, A. Tasneem, and T. M. Przytycka, "Co-evolutionary Analysis of Domains in Interacting Proteins Reveals Insights into Domain-Domain Interactions Mediating Protein-Protein Interactions," Journal of Molecular Biology, vol. 362, no. 4, pp. 861-875, Sep. 2006, doi: 10.1016/j.jmb.2006.07.072.

[16] S. E. Schelhorn, T. Lengauer, and M. Albrecht, "An integrative approach for predicting interactions of protein regions," in Bioinformatics, Aug. 2008, vol. 24, no. 16, doi: 10.1093/bioinformatics/btn290.

[17] D. Han, H. S. Kim, J. Seo, and W. Jang, "A domain combination based probabilistic framework for protein-protein interaction prediction.," Genome informatics. 
International Conference on Genome Informatics, vol. 14, 2003, doi: 10.11234/gi1990.14.250.

[18] K. Dick and J. Green, "Comparison of sequence- and structure-based protein-protein interaction sites," 2016, doi: 10.1109/EMBSISC.2016.7508605.

[19] Y. Lecun and Y. Bengio, "Pattern Recognition and Neural Networks," NJ, 1995. [Online]. Available: http://yann.lecun.com/exdb/publis/pdf/lecun-bengio-95b.pdf.

[20] Y. Lecun and Y. Bengio, "Convolutional Networks for Images, Speech, and Time Series Variable-Size Convolutional Networks: SDNNs," Processing, vol. 3361, no. 10, 2010.

[21] P. Cunningham, M. Cord, and S. J. Delany, "Supervised learning," in Cognitive Technologies, 2008, pp. 21-49, doi: 10.1007/978-3-540-75171-7_2.

[22] Z. Ghahramani, "Unsupervised learning," Lecture Notes in Computer Science (including subseries Lecture Notes in Artificial Intelligence and Lecture Notes in Bioinformatics), vol. 3176, pp. 72-112, 2004, doi: 10.1007/978-3-540-28650-9_5.

[23] J. Yang and J. Yang, "Image Pattern Recognition," in Encyclopedia of Biometrics, Boston, MA: Springer US, 2009, pp. 726-729.

[24] R. M. Haralick and L. G. Shapiro, "Image segmentation techniques.," Computer Vision, Graphics, \& Image Processing, vol. 29, no. 1, pp. 100-132, Jan. 1985, doi: 10.1016/S0734-189X(85)90153-7.

[25] X. Xie, K.-M. Lam, and Q. Dai, "Image Segmentation," in Encyclopedia of Biometrics, Boston, MA: Springer US, 2009, pp. 729-730.

[26] "Metrics to Evaluate your Semantic Segmentation Model | Towards Data Science." https://towardsdatascience.com/metrics-to-evaluate-your-semantic-segmentationmodel-6bcb99639aa2 (accessed May 04, 2021).

[27] P. Jaccard, "THE Distribution OF THE FLORA iN THE ALPINE ZONE.," New Phytologist, vol. 11, no. 2, pp. 37-50, Feb. 1912, doi: 10.1111/j.14698137.1912.tb05611.x. 
[28] L. R. Dice, "Measures of the Amount of Ecologic Association Between Species," Ecology, vol. 26, no. 3, pp. 297-302, Jul. 1945, doi: 10.2307/1932409.

[29] Willem, "Dice-Score vs IoU," Stack Exchange, Nov. 13, 2017. https://stats.stackexchange.com/questions/273537/f1-dice-score-vsiou/276144\#276144.

[30] P. J. Werbos, "Beyond Regression: New Tools for Prediction and Analysis in the Behavioral Sciences," PhD Thesis, Harvard U., vol. PhD thesis, no. January 1974, p. 454, 1974, [Online]. Available: http://www.citeulike.org/group/1938/article/1055600.

[31] A. K. Jain, J. Mao, and K. M. Mohiuddin, "Artificial neural networks: A tutorial," Computer, vol. 29, no. 3. pp. 31-44, Mar. 1996, doi: 10.1109/2.485891.

[32] Y. LeCun, P. Haffner, L. Bottou, and Y. Bengio, "Object recognition with gradientbased learning," in Lecture Notes in Computer Science (including subseries Lecture Notes in Artificial Intelligence and Lecture Notes in Bioinformatics), 1999, vol. 1681, doi: 10.1007/3-540-46805-6_19.

[33] K. O'Shea and R. Nash, "An Introduction to Convolutional Neural Networks," Nov. 2015, [Online]. Available: https://arxiv.org/abs/1511.08458v1.

[34] A. F. Agarap, "Deep Learning using Rectified Linear Units (ReLU)," arXiv, Mar. 2018, Accessed: May 04, 2021. [Online]. Available: http://arxiv.org/abs/1803.08375.

[35] F. H. Eeckman and W. J. Freman, "The sigmoid nonlinearity in neural computation. An experimental approach," 2008, doi: 10.1063/1.36270.

[36] J. Westermarck, J. Ivaska, and G. L. Corthals, "Identification of protein interactions involved in cellular signaling," Molecular and Cellular Proteomics, vol. 12, no. 7. American Society for Biochemistry and Molecular Biology Inc., pp. 1752-1763, Jul. 01, 2013, doi: 10.1074/mcp.R113.027771.

[37] N. Sahni et al., "Widespread macromolecular interaction perturbations in human genetic disorders," Cell, vol. 161, no. 3, pp. 647-660, Apr. 2015, doi: 10.1016/j.cell.2015.04.013. 
[38] I. Petta, S. Lievens, C. Libert, J. Tavernier, and K. de Bosscher, "Modulation of protein-protein interactions for the development of novel therapeutics," Molecular Therapy, vol. 24, no. 4. Nature Publishing Group, pp. 707-718, Apr. 01, 2016, doi: 10.1038/mt.2015.214.

[39] H. C. Lu, A. Fornili, and F. Fraternali, "Protein-Protein interaction networks studies and importance of 3D structure knowledge," Expert Review of Proteomics, vol. 10, no. 6. Taylor \& Francis, pp. 511-520, 2013, doi: 10.1586/14789450.2013.856764.

[40] M. Higurashi, T. Ishida, and K. Kinoshita, "PiSite: A database of protein interaction sites using multiple binding states in the PDB," Nucleic Acids Research, vol. 37, no. SUPPL. 1, pp. D360-D364, Jan. 2009, doi: 10.1093/nar/gkn659.

[41] T. C. Northey, A. BarešiÄ ‡, and A. C. R. Martin, "IntPred: A structure-based predictor of protein-protein interaction sites," Bioinformatics, vol. 34, no. 2, pp. 223-229, Jan. 2018, doi: 10.1093/bioinformatics/btx585.

[42] Q. C. Zhang et al., "Structure-based prediction of protein-protein interactions on a genome-wide scale," Nature, vol. 490, no. 7421, pp. 556-560, Oct. 2012, doi: 10.1038/nature11503.

[43] Y. Li and L. Ilie, "SPRINT: Ultrafast protein-protein interaction prediction of the entire human interactome," BMC Bioinformatics, vol. 18, no. 1, 2017, doi: 10.1186/s12859017-1871-x.

[44] C. Jary, "Improving the Protein-Protein Interaction Prediction Engine (PIPE) with Protein Physicochemical Properties," 2019. Accessed: May 04, 2021. [Online].

[45] S. Pitre et al., "Global investigation of protein-protein interactions in yeast Saccharomyces cerevisiae using re-occurring short polypeptide sequences," Nucleic Acids Research, vol. 36, no. 13, pp. 4286-4294, Aug. 2008, doi: 10.1093/nar/gkn390.

[46] M. O. Dayhoff, R. M. Schwartz, and B. C. Orcutt, "A model of evolutionary change in proteins," in Atlas of protein sequence and structure, 1978. 
[47] W. R. Pearson, "Selecting the right similarity-scoring matrix," Current Protocols in Bioinformatics, vol. 43, no. SUPL.43, pp. 3.5.1-3.5.9, Oct. 2013, doi: 10.1002/0471250953.bi0305s43.

[48] C. Patulea, Targeted Optimization of Computational and Classification Performance of a Protein-Protein Interaction Predictor. 2011.

[49] V. Kumar, S. Mahato, A. Munshi, and M. Kulharia, "PPInS: a repository of proteinprotein interaction sitesbase," Scientific Reports, vol. 8, no. 1, pp. 1-9, Dec. 2018, doi: $10.1038 / s 41598-018-30999-1$.

[50] X. Li, L. Yang, X. Zhang, and X. Jiao, "Prediction of Protein-Protein Interactions Based on Domain," Computational and Mathematical Methods in Medicine, vol. 2019, 2019, doi: $10.1155 / 2019 / 5238406$.

[51] S. K. Ng, Z. Zhang, S. H. Tan, and K. Lin, "InterDom: A database of putative interacting protein domains for validating predicted protein interactions and complexes," Nucleic Acids Research, vol. 31, no. 1. Nucleic Acids Res, pp. 251-254, Jan. 01, 2003, doi: 10.1093/nar/gkg079.

[52] H. Wang, E. Segal, A. Ben-Hur, Q. R. Li, M. Vidal, and D. Koller, "InSite: A computational method for identifying protein-protein interaction binding sites on a proteome-wide scale," Genome Biology, vol. 8, no. 9, pp. 1-18, Sep. 2007, doi: 10.1186/gb-2007-8-9-r192.

[53] J. Zhang and L. Kurgan, "SCRIBER: Accurate and partner type-specific prediction of protein-binding residues from proteins sequences," in Bioinformatics, Jul. 2019, vol. 35, no. 14, pp. i343-i353, doi: 10.1093/bioinformatics/btz324.

[54] M. Zeng, F. Zhang, F. X. Wu, Y. Li, J. Wang, and M. Li, "Protein-protein interaction site prediction through combining local and global features with deep neural networks," Bioinformatics, vol. 36, no. 4, pp. 1114-1120, Feb. 2020, doi: 10.1093/bioinformatics/btz699. 
[55] B. Zhang, J. Li, L. Quan, Y. Chen, and Q. Lü, "Sequence-based prediction of proteinprotein interaction sites by simplified long short-term memory network," Neurocomputing, vol. 357, pp. 86-100, Sep. 2019, doi: 10.1016/j.neucom.2019.05.013.

[56] Y. Li, G. B. Golding, and L. Ilie, "DELPHI: accurate deep ensemble model for protein interaction sites prediction," Bioinformatics, Aug. 2020, doi: 10.1093/bioinformatics/btaa750.

[57] A. Bateman et al., "UniProt: A hub for protein information," Nucleic Acids Research, vol. 43, no. D1, pp. D204-D212, Jan. 2015, doi: 10.1093/nar/gku989.

[58] H. Lee, M. Deng, F. Sun, and T. Chen, "An integrated approach to the prediction of domain-domain interactions," BMC Bioinformatics, vol. 7, no. 1, pp. 1-15, May 2006, doi: $10.1186 / 1471-2105-7-269$.

[59] K. S. Guimarães, R. Jothi, E. Zotenko, and T. M. Przytycka, "Predicting domaindomain interactions using a parsimony approach," Genome Biology, vol. 7, no. 11, pp. 1-14, Nov. 2006, doi: 10.1186/gb-2006-7-11-r104.

[60] R. Riley, C. Lee, C. Sabatti, and D. Eisenberg, "Inferring protein domain interactions from databases of interacting proteins.," Genome biology, vol. 6, no. 10, pp. 1-17, Sep. 2005, doi: 10.1186/gb-2005-6-10-r89.

[61] Galarnyk Michael, "Understanding Boxplots," Towards Data Science, Sep. 12, 2018. https://towardsdatascience.com/understanding-boxplots-5e2df7bcbd51.

[62] C. Stark, B. J. Breitkreutz, T. Reguly, L. Boucher, A. Breitkreutz, and M. Tyers, "BioGRID: a general repository for interaction datasets.," Nucleic acids research, vol. 34, no. Database issue, pp. D535-D539, Jan. 2006, doi: 10.1093/nar/gkj109.

[63] R. Mosca, A. Céol, A. Stein, R. Olivella, and P. Aloy, "3did: A catalog of domain-based interactions of known three-dimensional structure," Nucleic Acids Research, vol. 42, no. D1, pp. D374-D379, Jan. 2014, doi: 10.1093/nar/gkt887. 
[64] A. Bateman et al., "The Pfam protein families database," Nucleic Acids Research, vol. 32, no. DATABASE ISS., pp. D138-D141, Jan. 2004, doi: 10.1093/nar/gkh121.

[65] "Amazon EC2 M5 Instances - general purpose compute workloads." https://aws.amazon.com/ec2/instance-types/m5/ (accessed May 25, 2021).

[66] S. Okamura, "GridSearchCV for Beginners," Towards Data Science, Dec. 28, 2020. https://towardsdatascience.com/gridsearchcv-for-beginners-db48a90114ee.

[67] A. Mueller and Venkat, "sklearn.model_selection.GridSearchCV," scikit-learn.org, Oct. 25, 2015.2 https://scikitlearn.org/stable/modules/generated/sklearn.model_selection.GridSearchCV.html.

[68] Paul E. Black, "greedy algorithm," Dictionary of Algorithms and Data Structures, Feb. 02, 2005. https://xlinux.nist.gov/dads/HTML/greedyalgo.html.

[69] J. C. Olivo-Marin, "Extraction of spots in biological images using multiscale products," Pattern Recognition, vol. 35, no. 9, pp. 1989-1996, Sep. 2002, doi: 10.1016/S00313203(01)00127-3.

[70] E. S. Gedraite and M. Hadad, "Investigation on the effect of a Gaussian Blur in image filtering and segmentation | IEEE Conference Publication | IEEE Xplore," in Proceedings ELMAR-2011, 2011, pp. 393-396, Accessed: May 26, 2021. [Online]. Available: https://ieeexplore.ieee.org/abstract/document/6044249.

[71] I. Culjak, D. Abram, T. Pribanic, H. Dzapo, and M. Cifrek, "A brief introduction to OpenCV," 2012.

[72] C. Chen, G. Li, R. Xu, T. Chen, M. Wang, and L. Lin, "Clusternet: Deep hierarchical cluster network with rigorously rotation-invariant representation for point cloud analysis," in Proceedings of the IEEE Computer Society Conference on Computer Vision and Pattern Recognition, Jun. 2019, vol. 2019-June, pp. 4989-4997, doi: 10.1109/CVPR.2019.00513.

[73] R. LaLonde, D. Zhang, and M. Shah, "ClusterNet: Detecting Small Objects in Large Scenes by Exploiting Spatio-Temporal Information," Proceedings of the IEEE 
Computer Society Conference on Computer Vision and Pattern Recognition, pp. 40034012, Apr. 2017, Accessed: May 26, 2021. [Online]. Available: http://arxiv.org/abs/1704.02694.

[74] A. Shukla, G. S. Cheema, and S. Anand, "Semi-Supervised Clustering with Neural Networks," in Proceedings - 2020 IEEE 6th International Conference on Multimedia Big Data, BigMM 2020, Sep. 2020, pp. 152-161, doi: 10.1109/BigMM50055.2020.00030.

[75] W. Kim, A. Kanezaki, and M. Tanaka, "Unsupervised Learning of Image Segmentation Based on Differentiable Feature Clustering," IEEE Transactions on Image Processing, vol. 29, 2020, doi: 10.1109/TIP.2020.3011269.

[76] A. Kanezaki, "Unsupervised image segmentation by backpropagation," in ICASSP, IEEE International Conference on Acoustics, Speech and Signal Processing Proceedings, Sep. 2018, vol. 2018-April, pp. 1543-1547, doi: 10.1109/ICASSP.2018.8462533.

[77] X. Guo, X. Liu, E. Zhu, and J. Yin, "Deep Clustering with Convolutional Autoencoders," in Lecture Notes in Computer Science (including subseries Lecture Notes in Artificial Intelligence and Lecture Notes in Bioinformatics), 2017, vol. 10635 LNCS, pp. 373382, doi: 10.1007/978-3-319-70096-0_39.

[78] A. Krizhevsky, I. Sutskever, and G. E. Hinton, "ImageNet classification with deep convolutional neural networks," Communications of the ACM, vol. 60, no. 6, 2017, doi: $10.1145 / 3065386$.

[79] S. Ioffe and C. Szegedy, "Batch normalization: Accelerating deep network training by reducing internal covariate shift," in 32nd International Conference on Machine Learning, ICML 2015, Feb. 2015, vol. 1, pp. 448-456, Accessed: May 04, 2021. [Online]. Available: https://arxiv.org/abs/1502.03167v3.

[80] R. Achanta, A. Shaji, K. Smith, A. Lucchi, P. Fua, and S. Süsstrunk, "SLIC superpixels compared to state-of-the-art superpixel methods," IEEE Transactions on Pattern 
Analysis and Machine Intelligence, vol. 34, no. 11, 2012, doi: 10.1109/TPAMI.2012.120.

[81] S. Sudha, K. B. Jayanthi, C. Rajasekaran, and T. Sunder, "Segmentation of RoI in Medical Images Using CNN- A Comparative Study," in IEEE Region 10 Annual International Conference, Proceedings/TENCON, Oct. 2019, vol. 2019-October, pp. 767-771, doi: 10.1109/TENCON.2019.8929648.

[82] W. H. Kruskal and W. A. Wallis, "Use of Ranks in One-Criterion Variance Analysis," Journal of the American Statistical Association, vol. 47, no. 260, 1952, doi: 10.1080/01621459.1952.10483441.

[83] F. Wilcoxon, "Individual Comparisons by Ranking Methods," Biometrics Bulletin, vol. 1, no. 6 , p. 80 , Dec. 1945 , doi: $10.2307 / 3001968$.

[84] A. Paszke et al., "PyTorch: An imperative style, high-performance deep learning library," in Advances in Neural Information Processing Systems, 2019, vol. 32. 